\title{
Temperature- and Reagent-Controlled Complementary Syn- and Anti-Selective Enolboration-aldolization of Substituted Phenylacetates
}

Angela Y. Thomas, Tommy L. Walls III, Brionna N. Nelson, Stafford W. Primeaux, and Prem B. Chanda* Department of Chemistry and Physics, Southeastern Louisiana University

Hammond, LA 70402

Phone: (+) 1-985-549-2578

Email: prem.chanda@selu.edu

Supporting Information

Contents

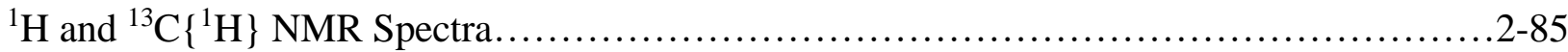

${ }^{1} \mathrm{H}$ and ${ }^{13} \mathrm{C}\left\{{ }^{1} \mathrm{H}\right\}$ NMR spectra of some representative crude compounds..................86-95 


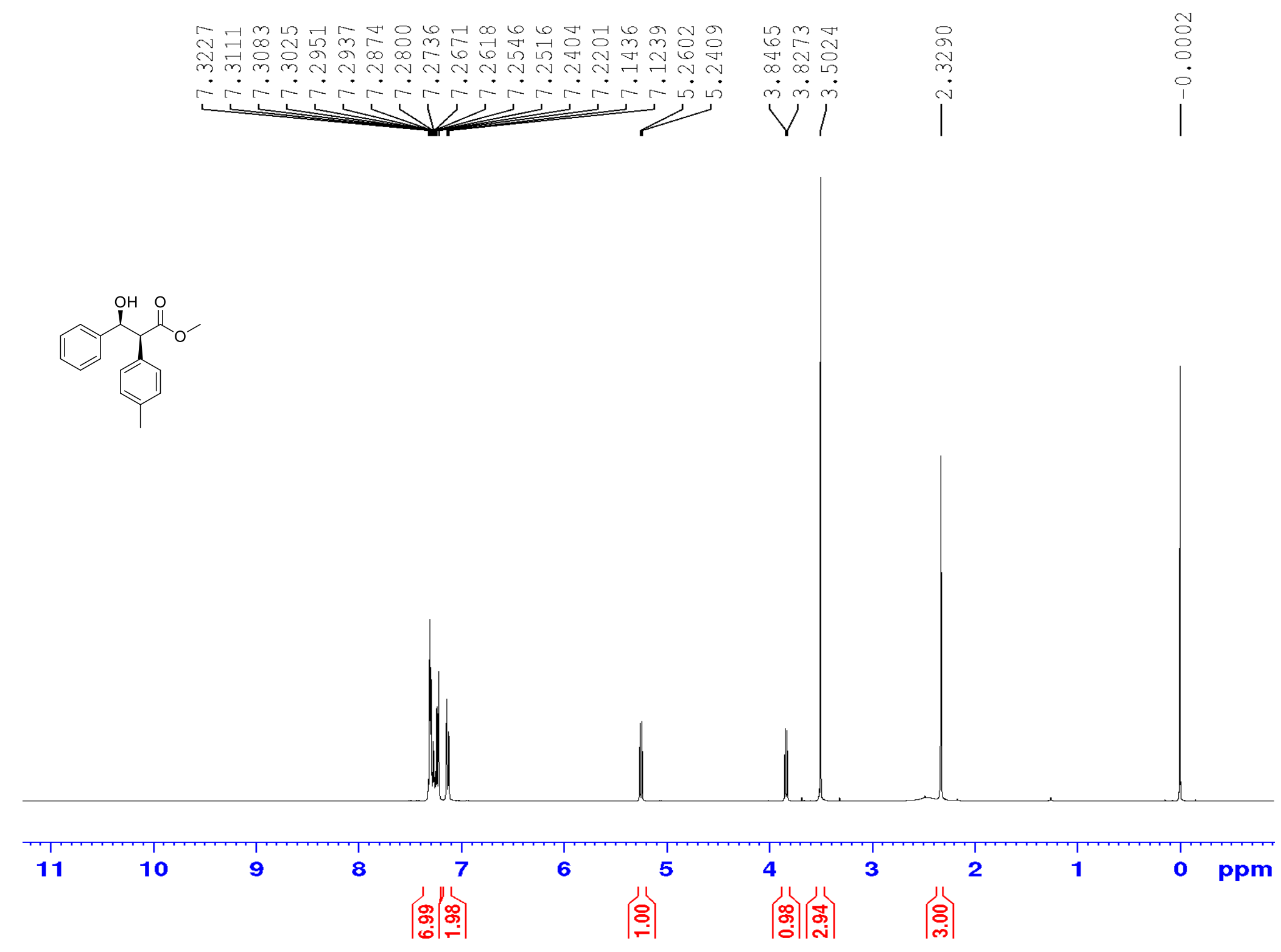

${ }^{1} \mathrm{H}$ NMR (400 MHz, $\mathrm{CDCl}_{3}$ ) Spectrum of syn-methyl 3-hydroxy-3-phenyl-2-p-tolylpropanoate (7a) 

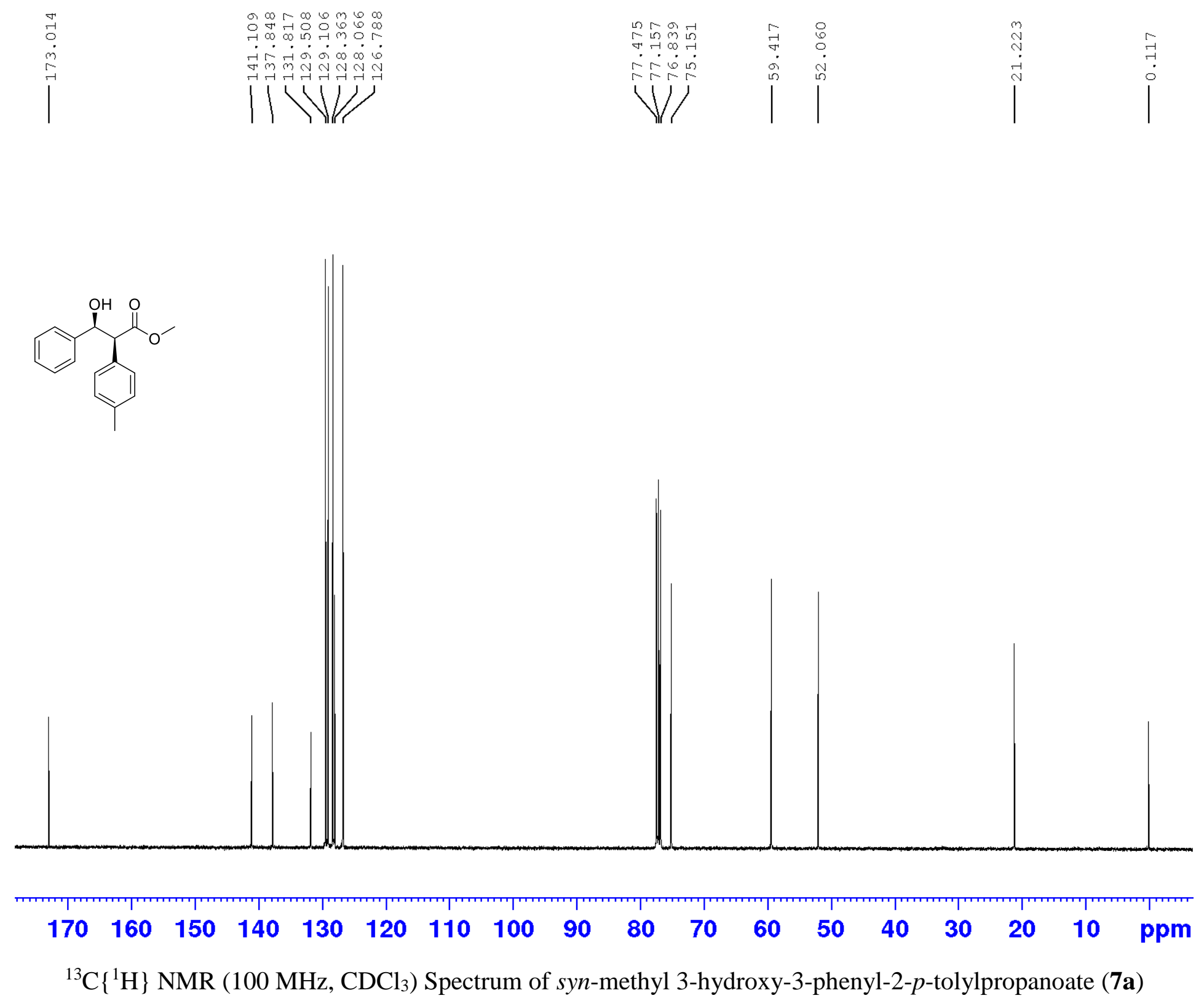


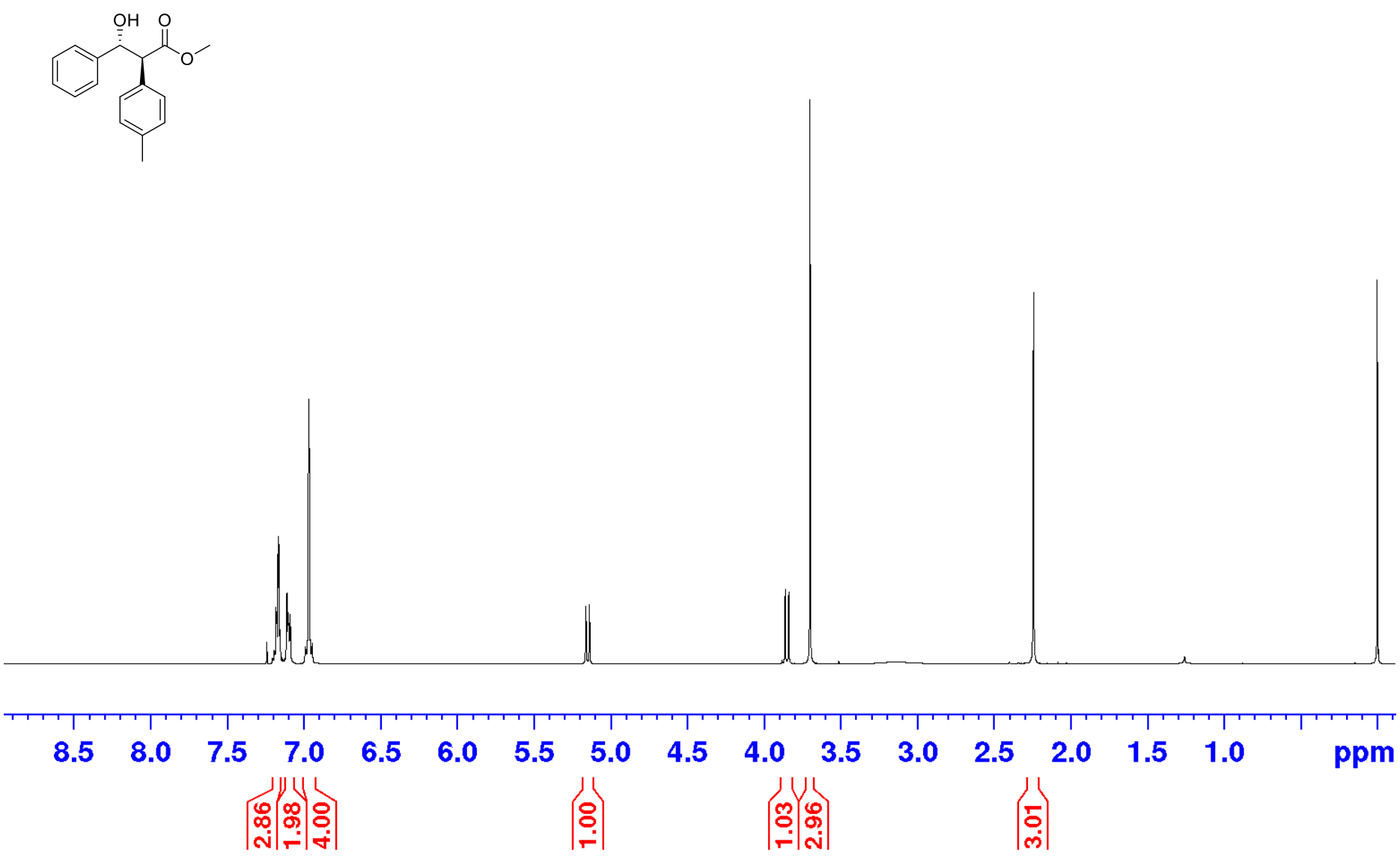

${ }^{1} \mathrm{H}$ NMR (400 MHz, $\mathrm{CDCl}_{3}$ ) Spectrum of anti-methyl 3-hydroxy-3-phenyl-2-p-tolylpropanoate (7a) 

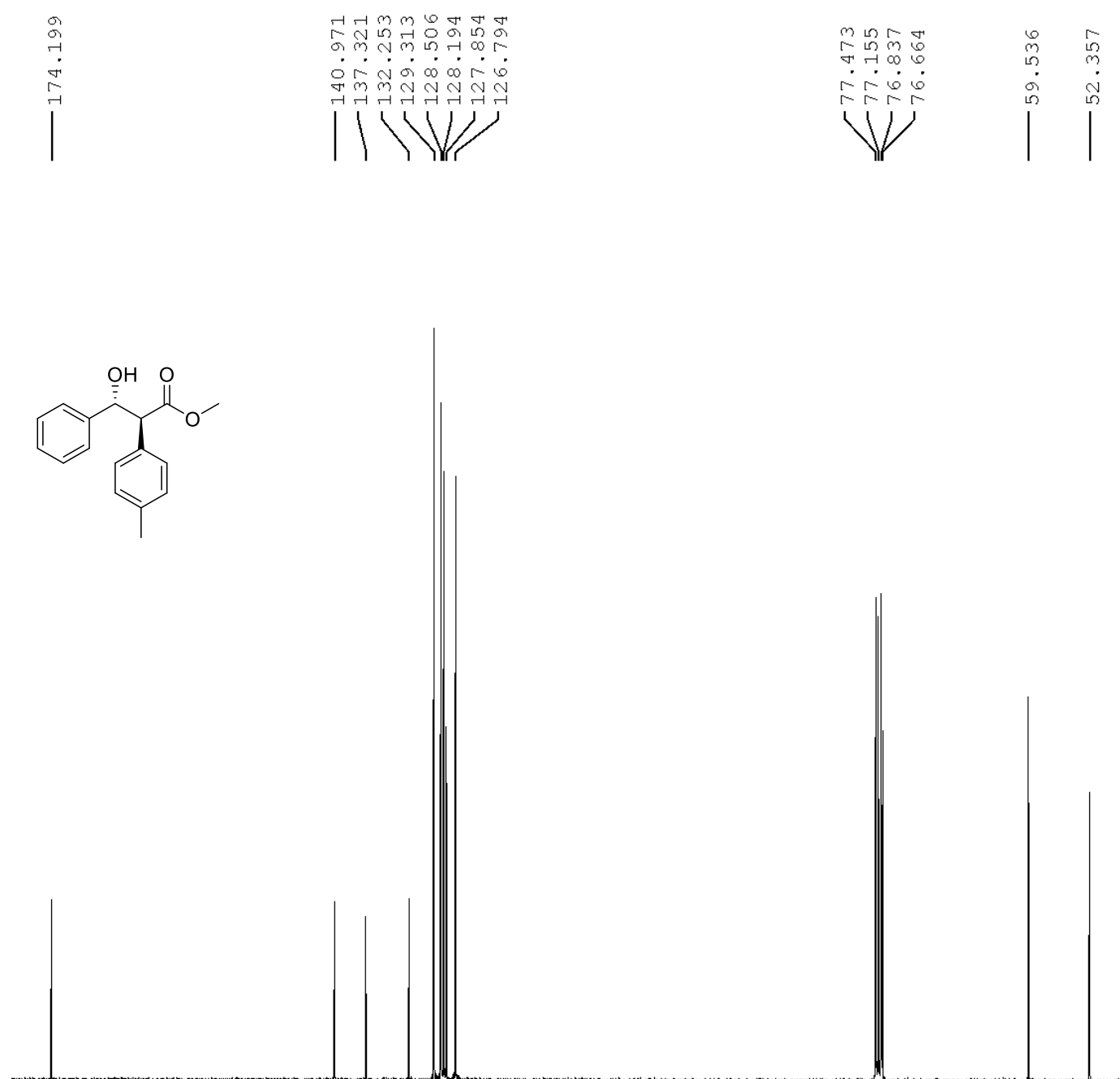

$\begin{array}{llllllll}170 & 160 & 150 & 140 & 130 & 120 & 110 & 100\end{array}$

${ }^{13} \mathrm{C}\left\{{ }^{1} \mathrm{H}\right\}$ NMR $\left(100 \mathrm{MHz}, \mathrm{CDCl}_{3}\right)$ Spectrum of anti-methyl 3-hydroxy-3-phenyl-2-p-tolylpropanoate (7a) 

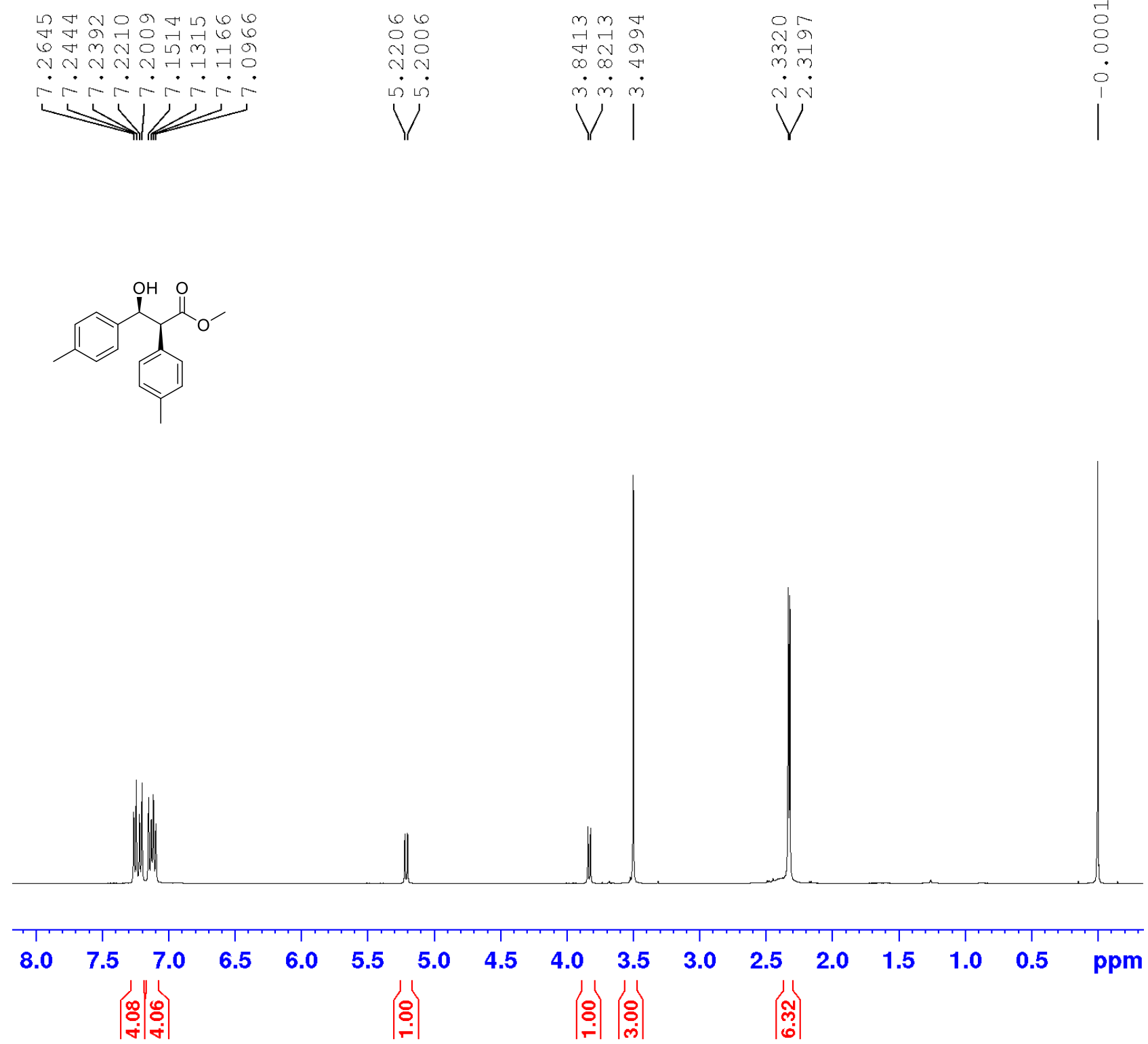

${ }^{1} \mathrm{H}$ NMR (400 MHz, $\mathrm{CDCl}_{3}$ ) Spectrum of syn-methyl 3-hydroxy-2, 3-dip-tolylpropanoate (7b) 

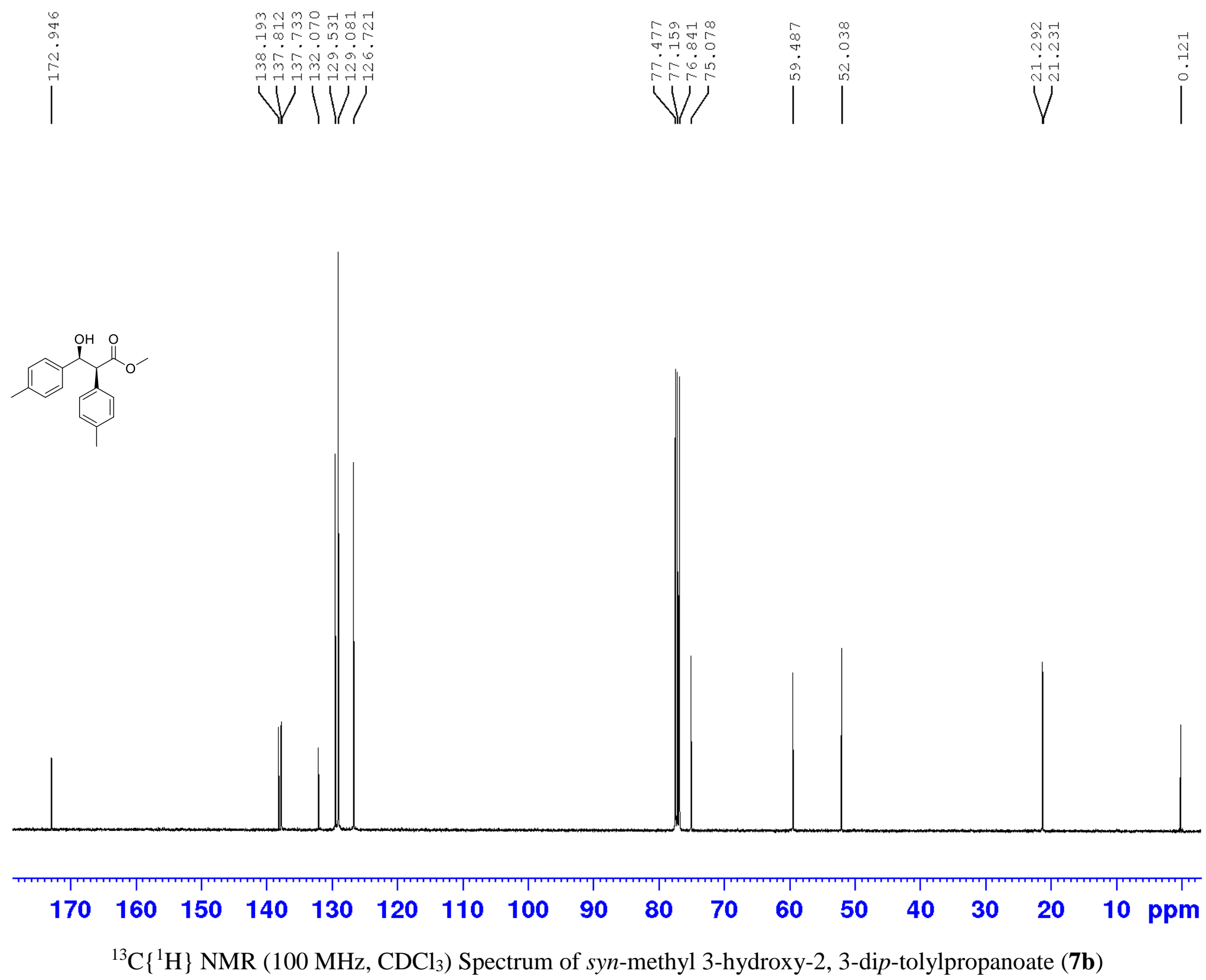


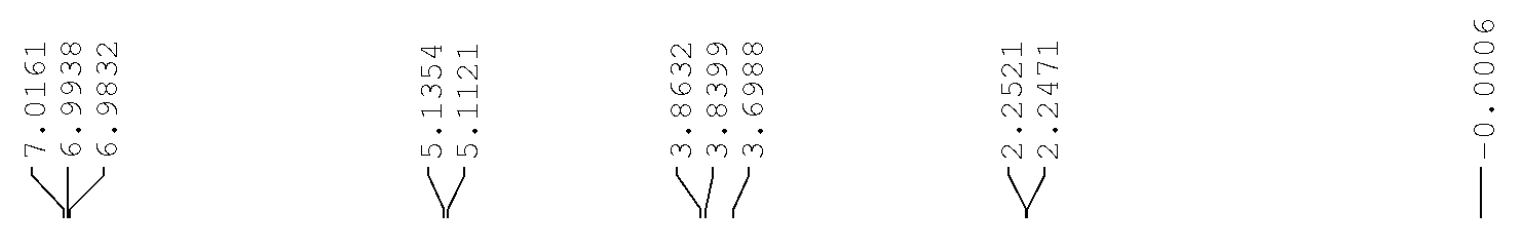
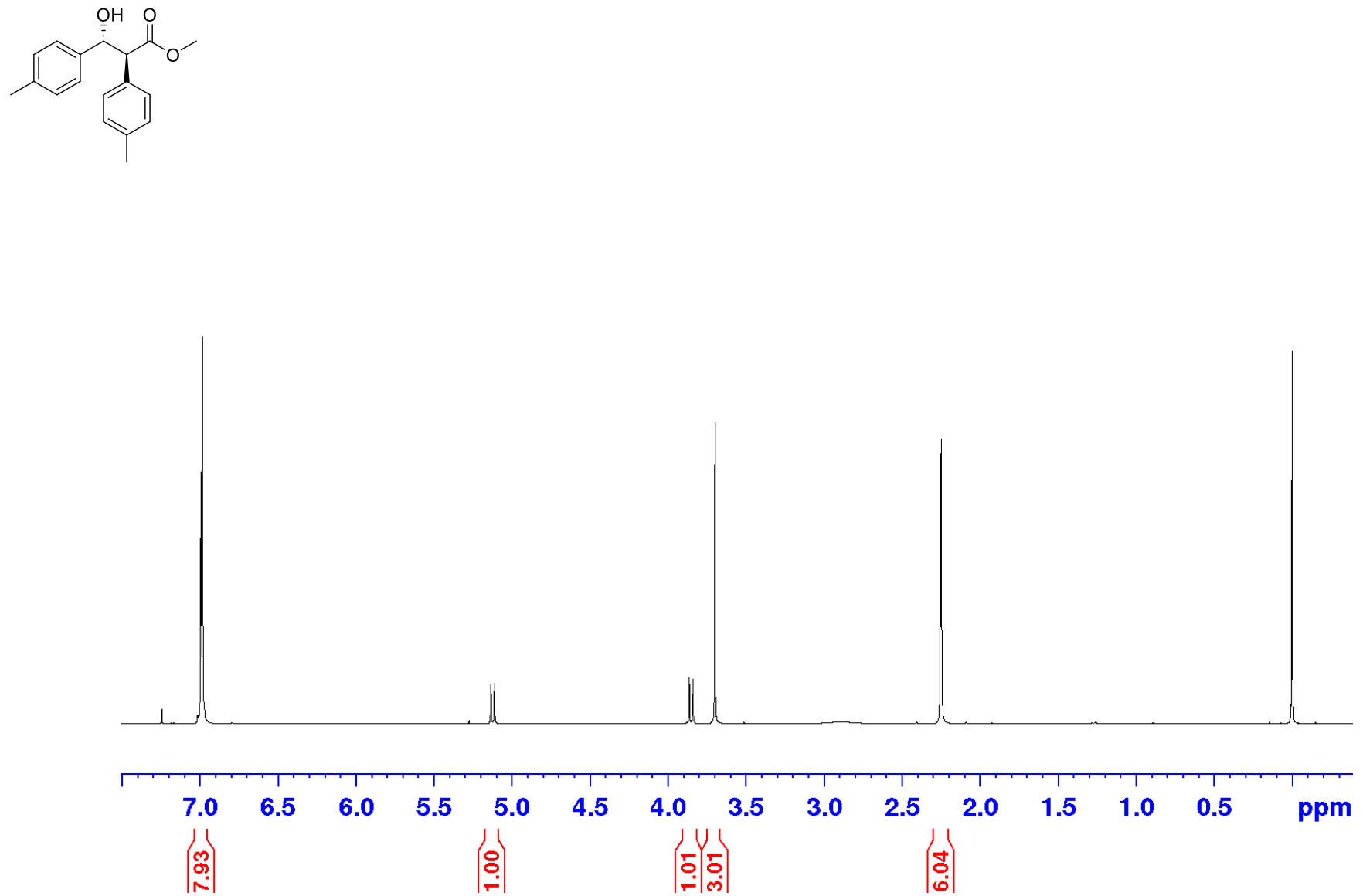

${ }^{1} \mathrm{H}$ NMR (400 MHz, $\mathrm{CDCl}_{3}$ ) Spectrum of anti-methyl 3-hydroxy-2, 3-dip-tolylpropanoate (7b) 


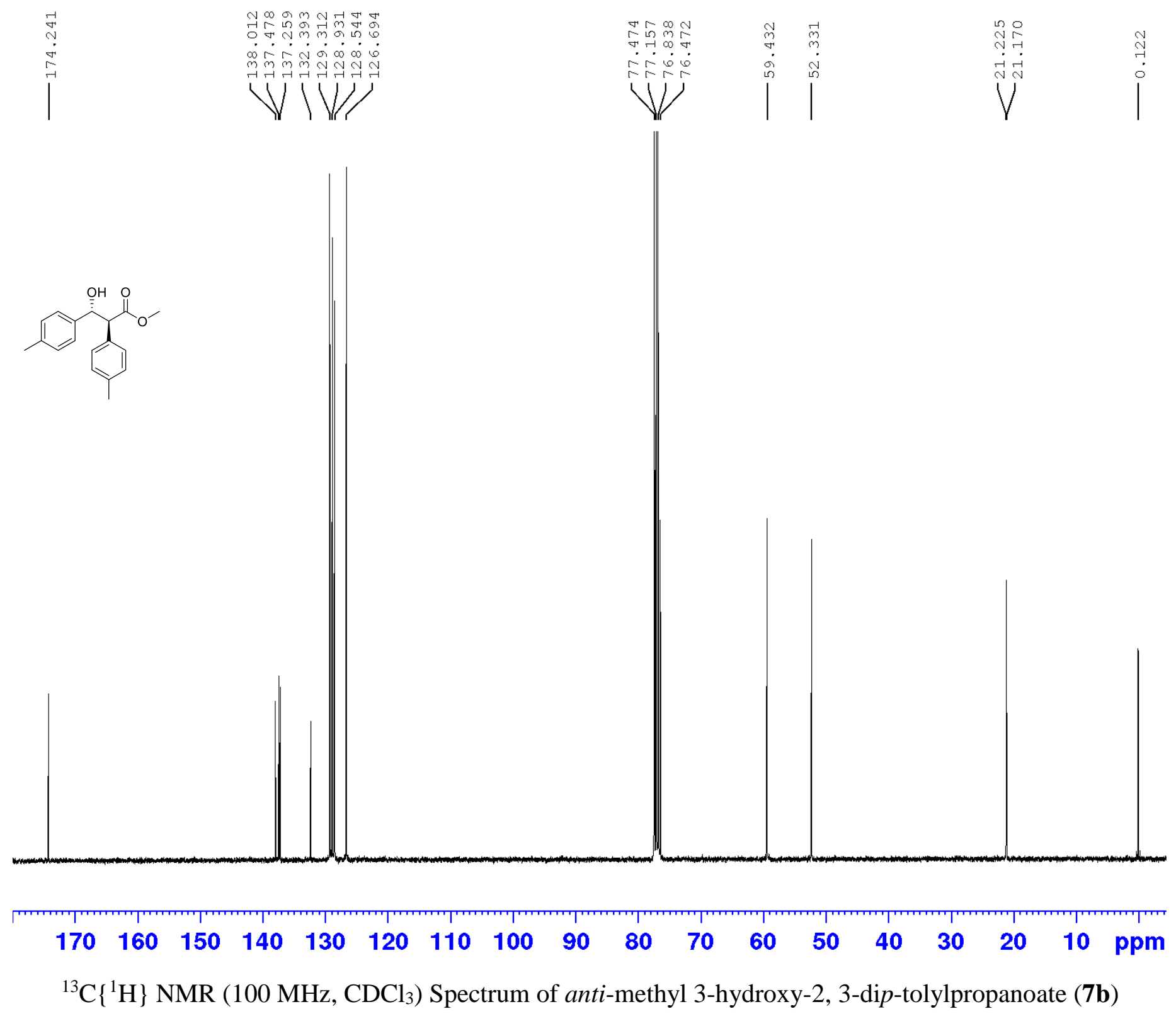



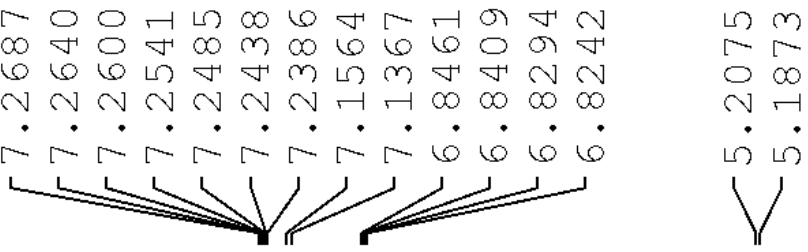

ก

$\checkmark \bigcirc \infty$

mim
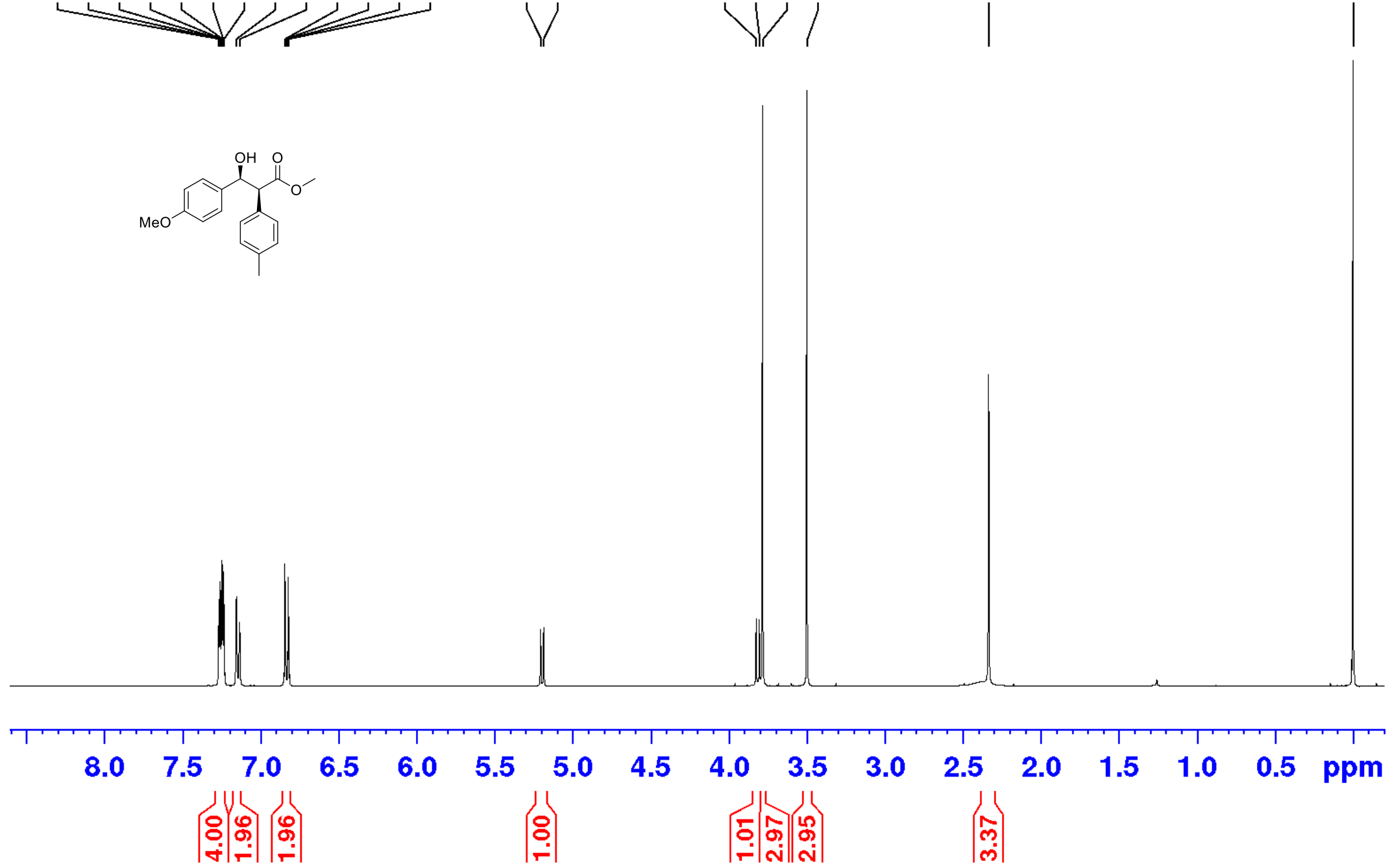

${ }^{1} \mathrm{H}$ NMR (400 MHz, $\mathrm{CDCl}_{3}$ ) Spectrum of syn-methyl 3-hydroxy-3-(4-methoxyphenyl)-2-p-tolylpropanoate (7c) 

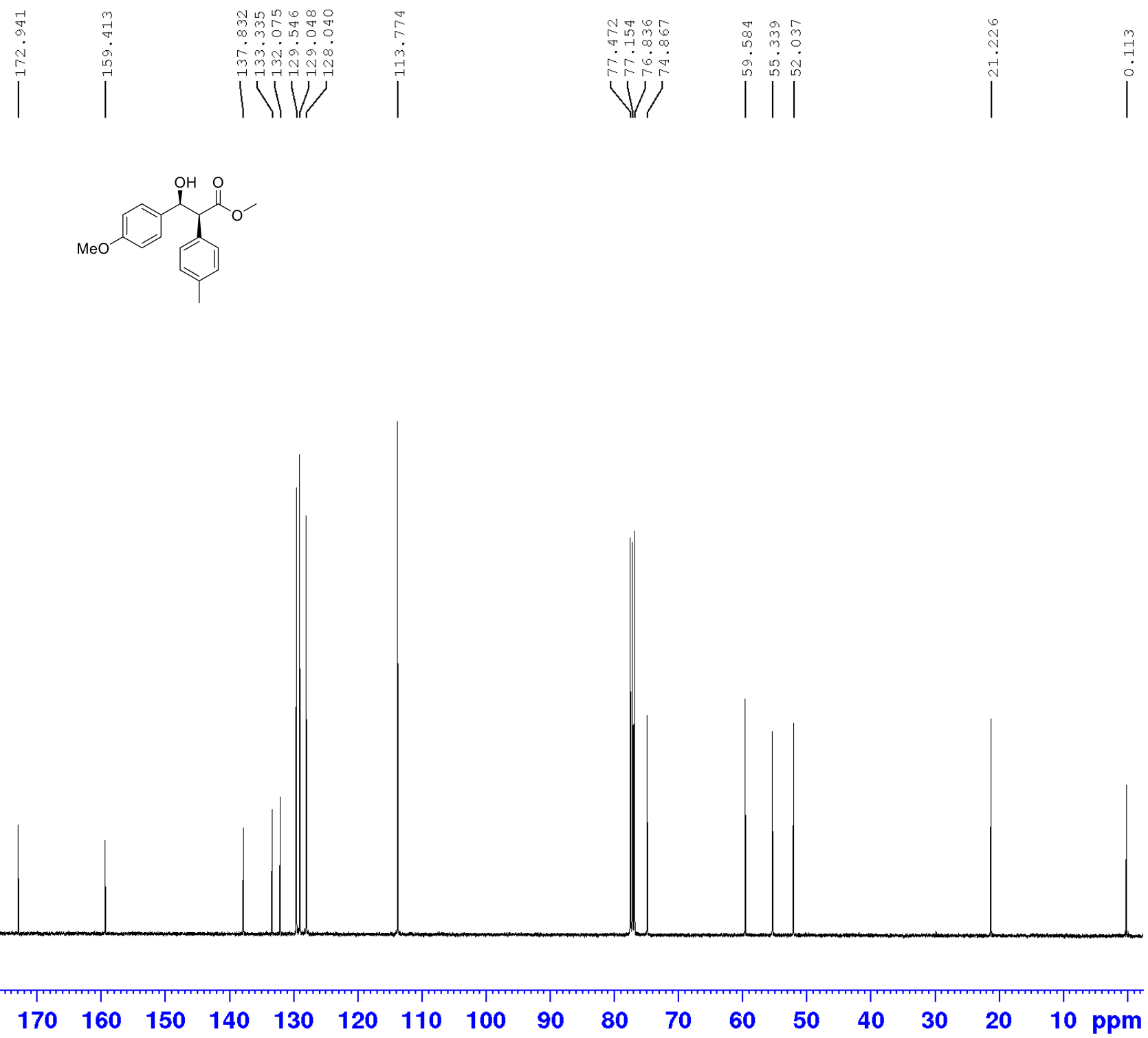

${ }^{13} \mathrm{C}\left\{{ }^{1} \mathrm{H}\right\}$ NMR (100 MHz, $\left.\mathrm{CDCl}_{3}\right)$ Spectrum of syn-methyl 3-hydroxy-3-(4-methoxyphenyl)-2-p-tolylpropanoate (7c) 


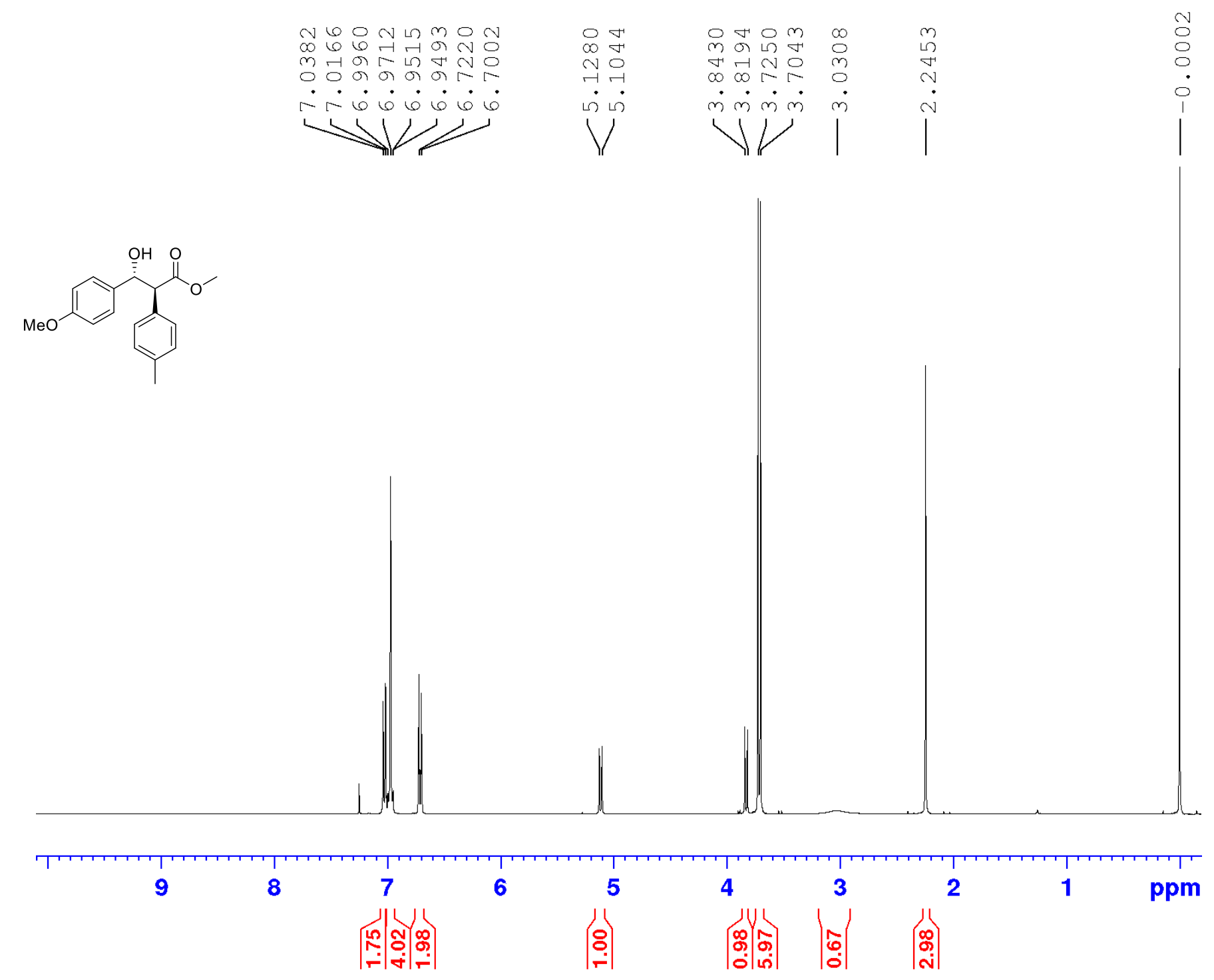

${ }^{1} \mathrm{H}$ NMR (400 MHz, $\mathrm{CDCl}_{3}$ ) Spectrum of anti-methyl 3-hydroxy-3-(4-methoxyphenyl)-2-p-tolylpropanoate (7c) 

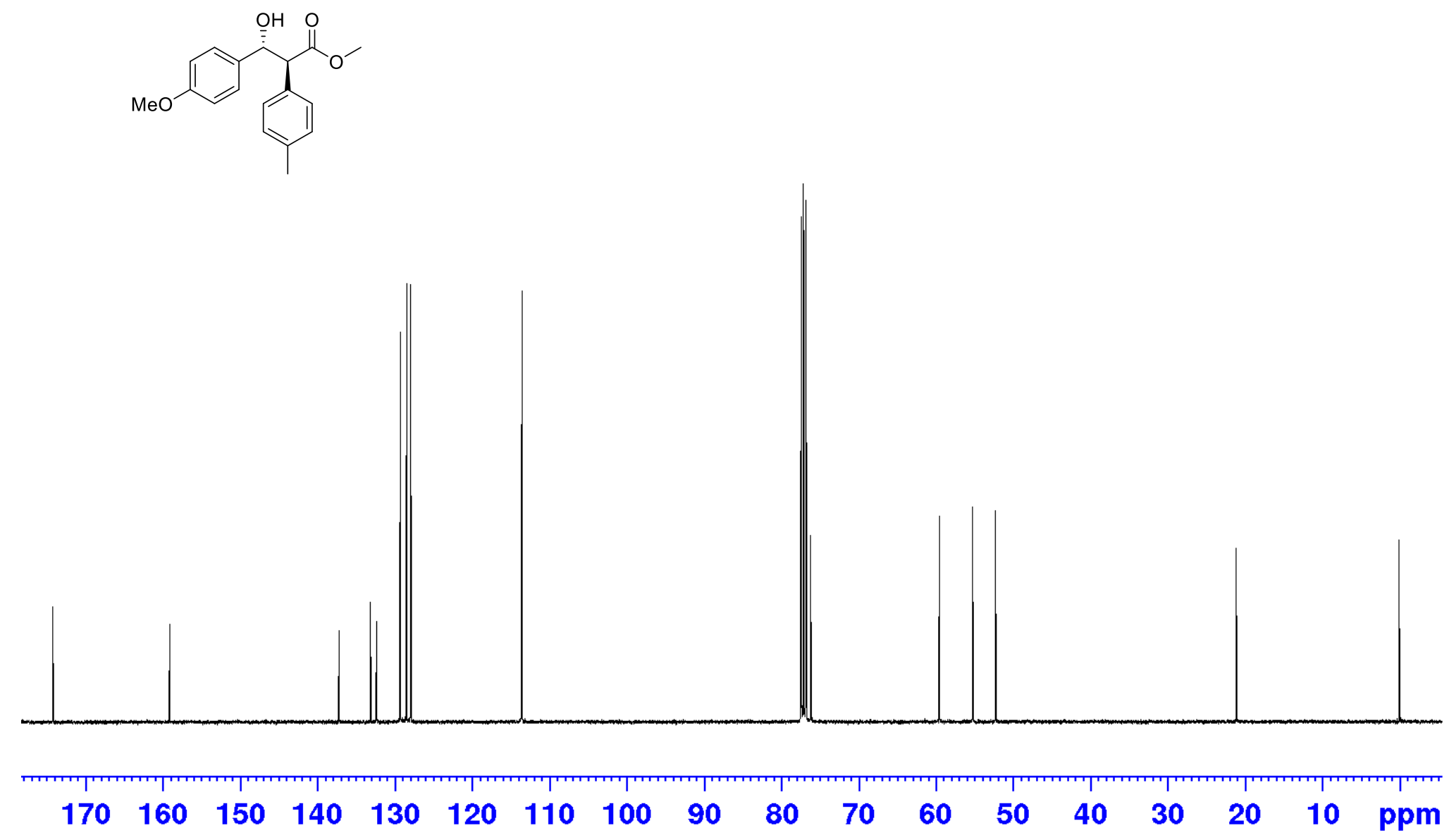

${ }^{13} \mathrm{C}\left\{{ }^{1} \mathrm{H}\right\}$ NMR (100 MHz, $\mathrm{CDCl}_{3}$ ) Spectrum of anti-methyl 3-hydroxy-3-(4-methoxyphenyl)-2-p-tolylpropanoate (7c) 

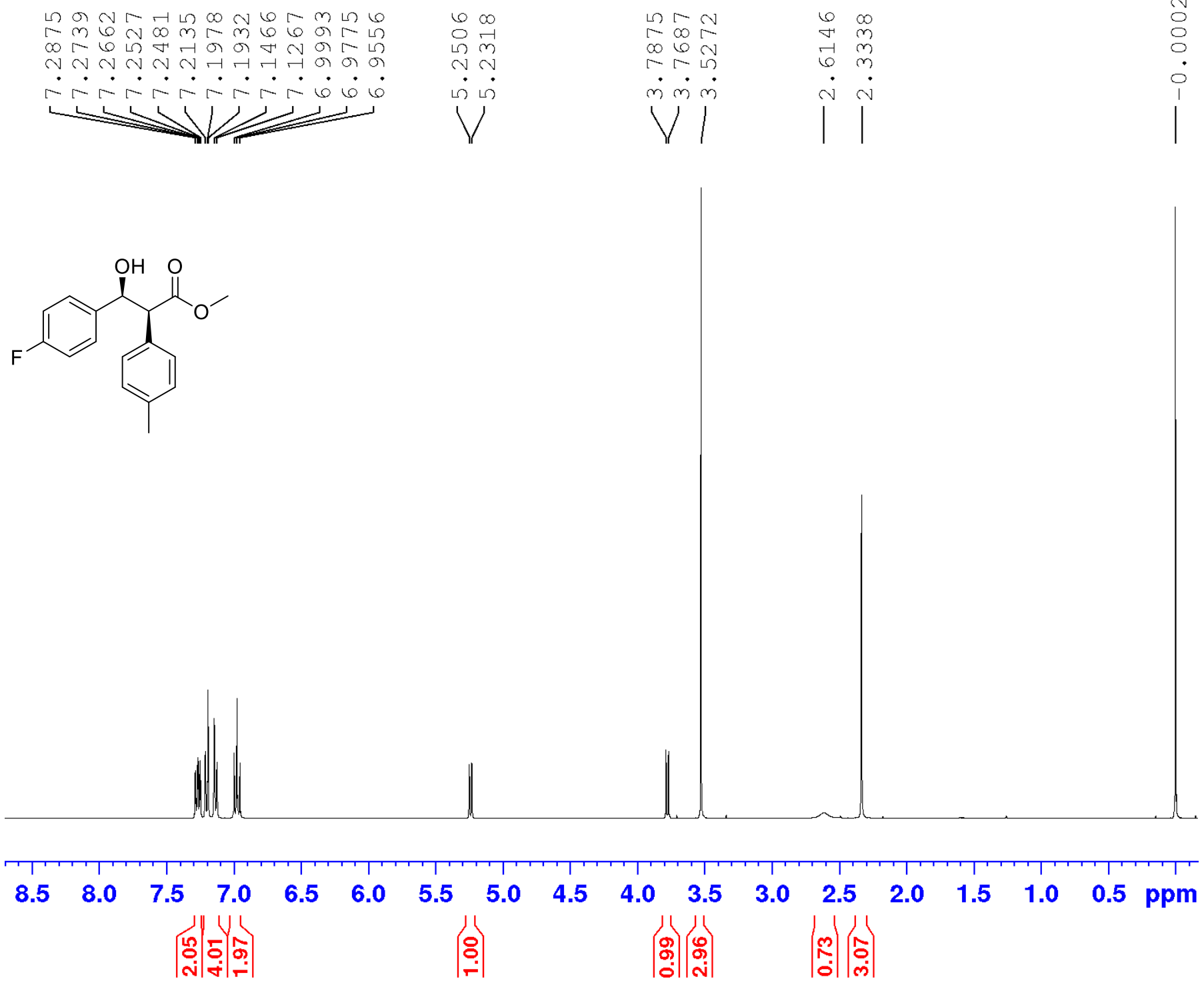

${ }^{1} \mathrm{H}$ NMR (400 MHz, $\mathrm{CDCl}_{3}$ ) Spectrum of syn-methyl 3-(4-fluorophenyl)-3-hydroxy-2-p-tolylpropanoate (7d) 


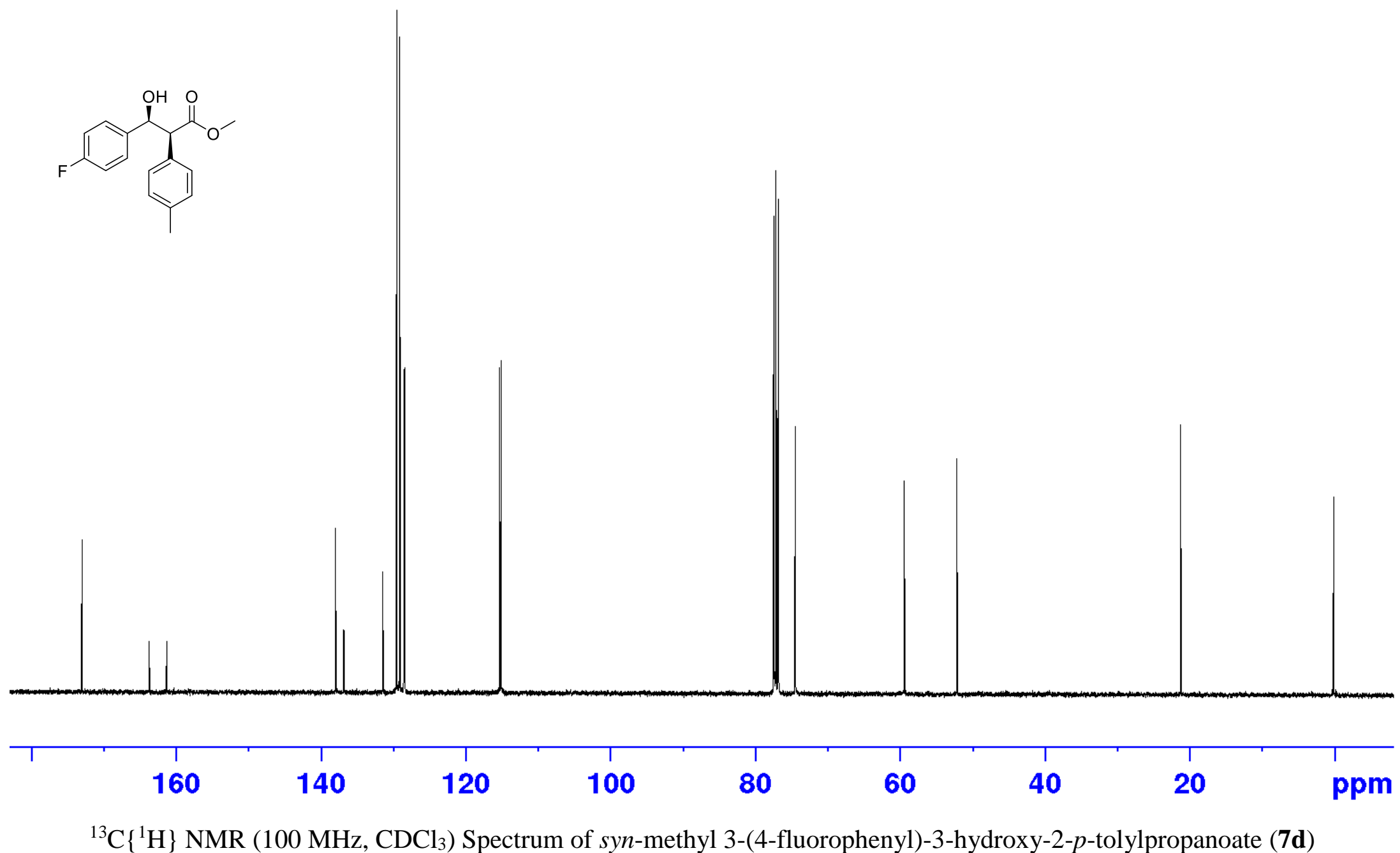



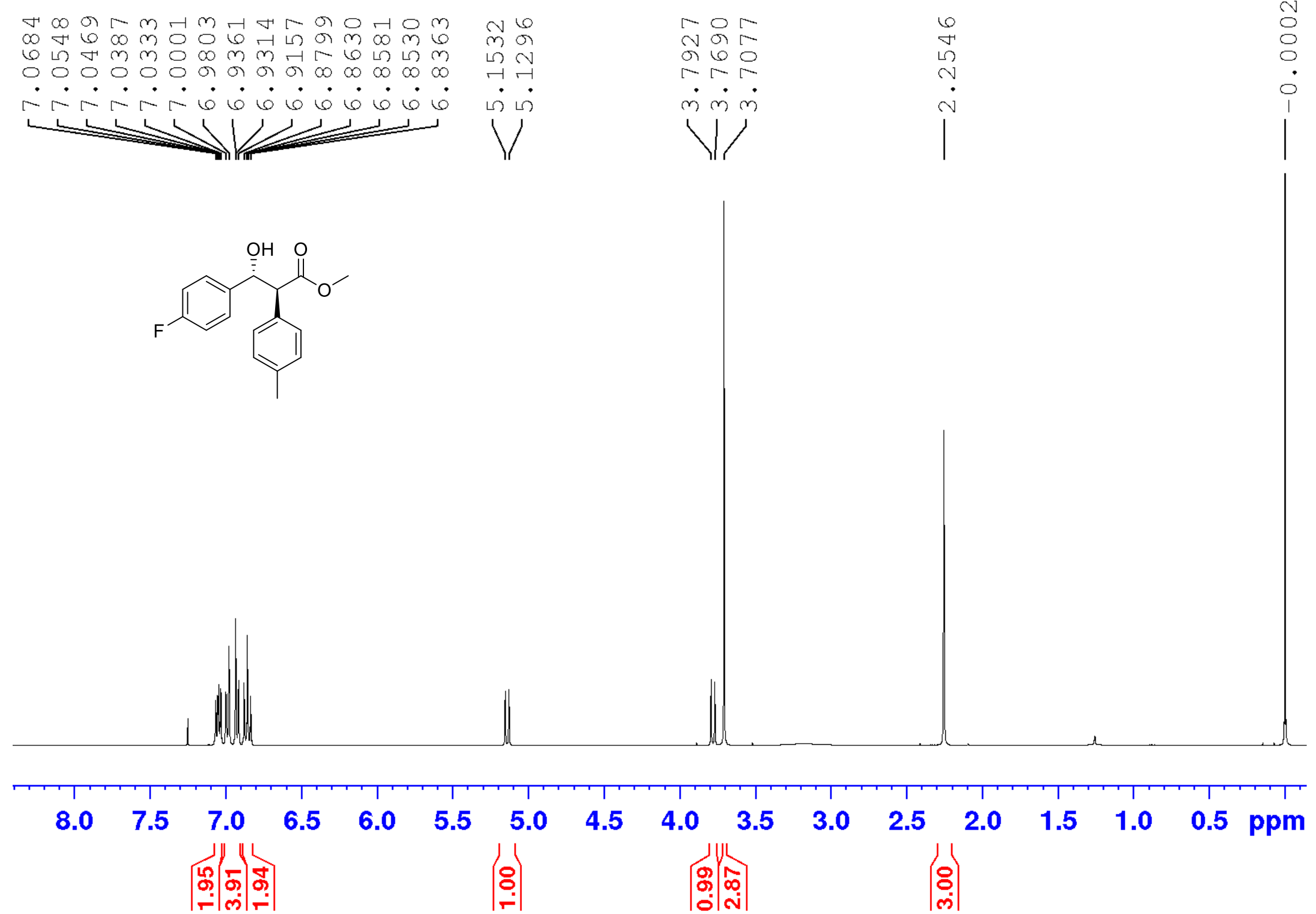

${ }^{1} \mathrm{H}$ NMR (400 MHz, $\mathrm{CDCl}_{3}$ ) Spectrum of anti-methyl 3-(4-fluorophenyl)-3-hydroxy-2-p-tolylpropanoate (7d) 


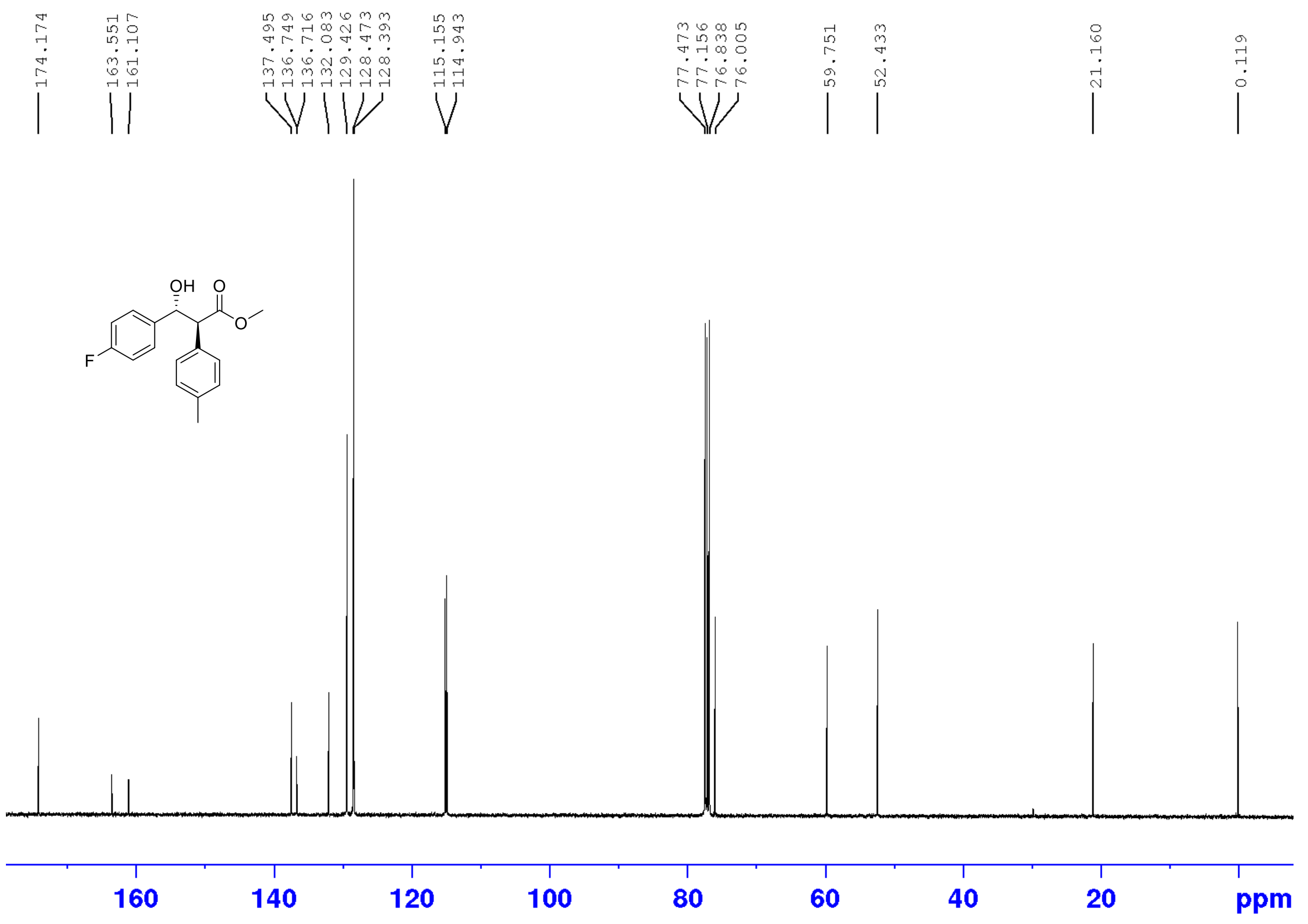

${ }^{13} \mathrm{C}\left\{{ }^{1} \mathrm{H}\right\}$ NMR (100 MHz, $\mathrm{CDCl}_{3}$ ) Spectrum of anti-methyl 3-(4-fluorophenyl)-3-hydroxy-2-p-tolylpropanoate (7d) 


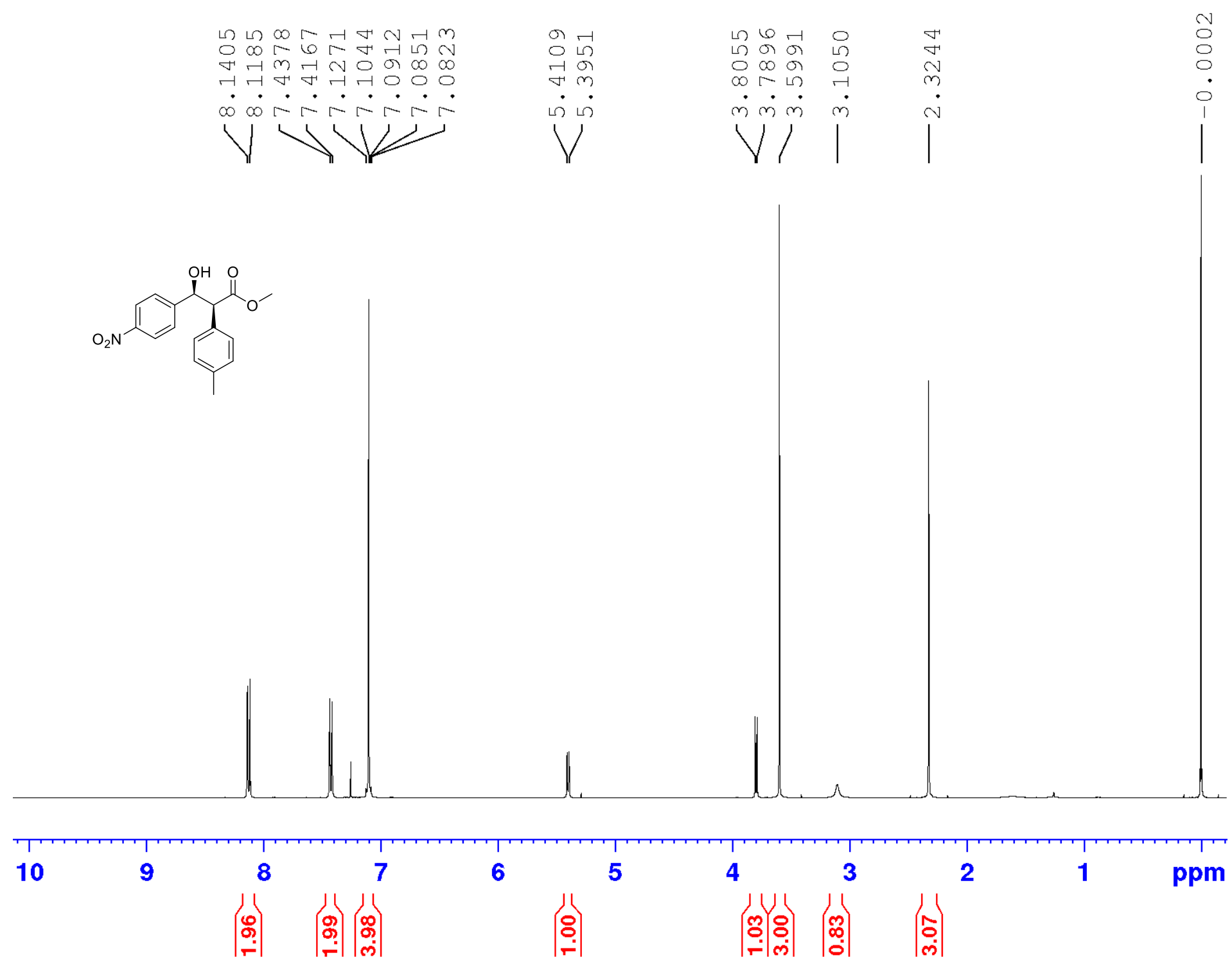

${ }^{1} \mathrm{H}$ NMR (400 MHz, $\mathrm{CDCl}_{3}$ ) Spectrum of syn-methyl 3-hydroxy-3-(4-nitrophenyl)-2-p-tolylpropanoate (7e) 

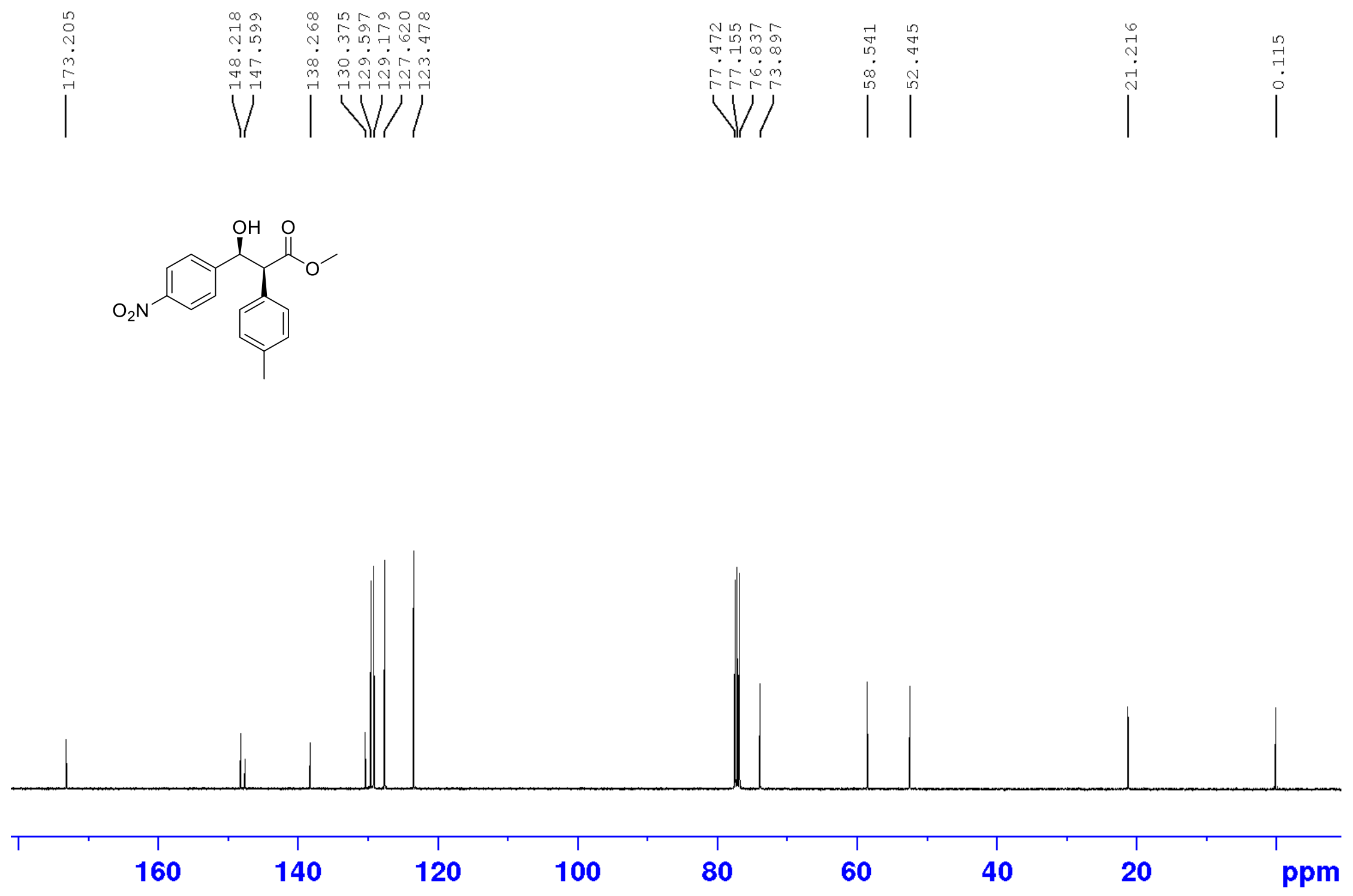

${ }^{13} \mathrm{C}\left\{{ }^{1} \mathrm{H}\right\}$ NMR $\left(100 \mathrm{MHz}, \mathrm{CDCl}_{3}\right)$ Spectrum of syn-methyl 3-hydroxy-3-(4-nitrophenyl)-2-p-tolylpropanoate (7e) 

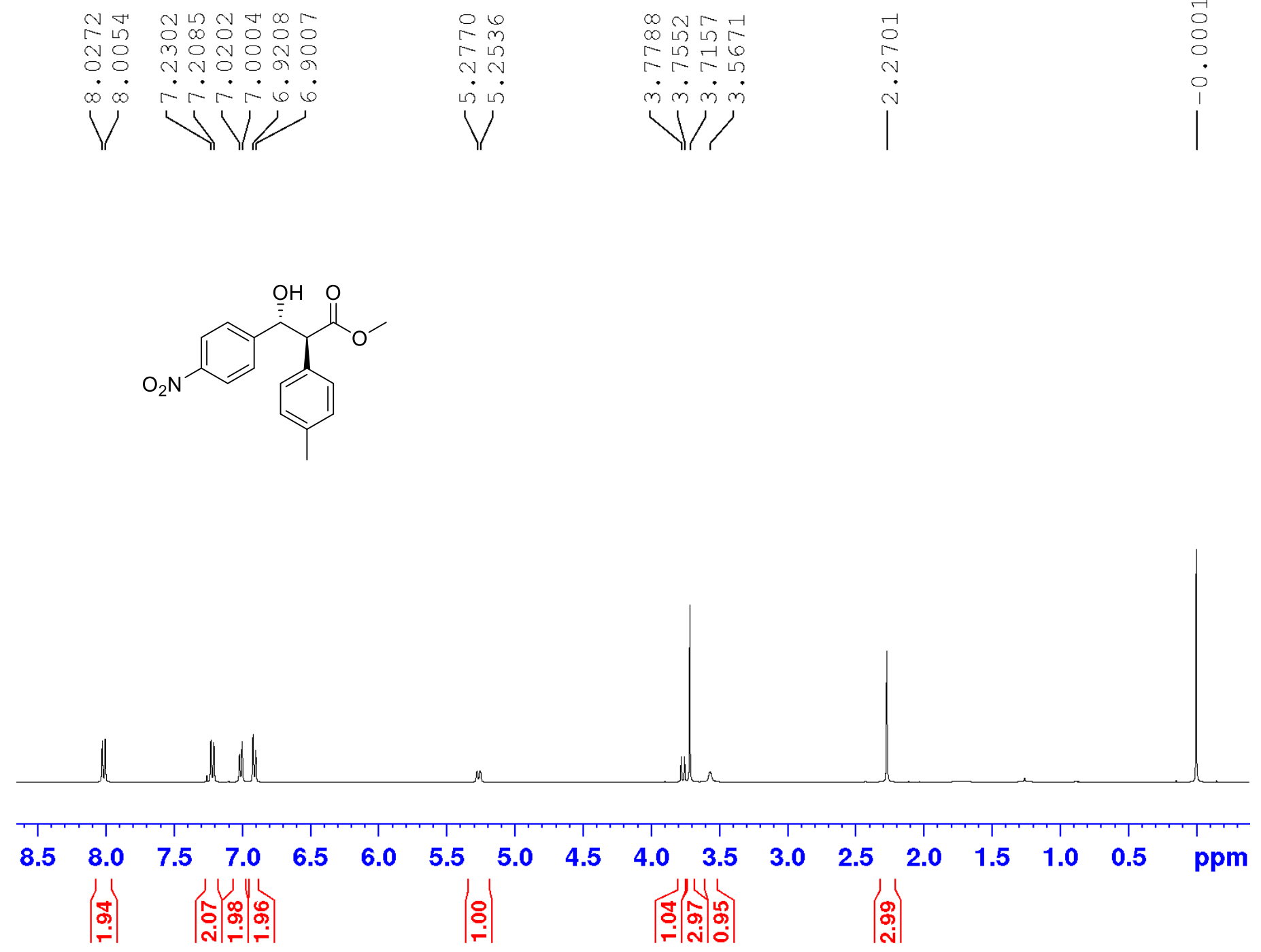

${ }^{1} \mathrm{H}$ NMR (400 MHz, $\mathrm{CDCl}_{3}$ ) Spectrum of anti-methyl 3-hydroxy-3-(4-nitrophenyl)-2-p-tolylpropanoate (7e) 

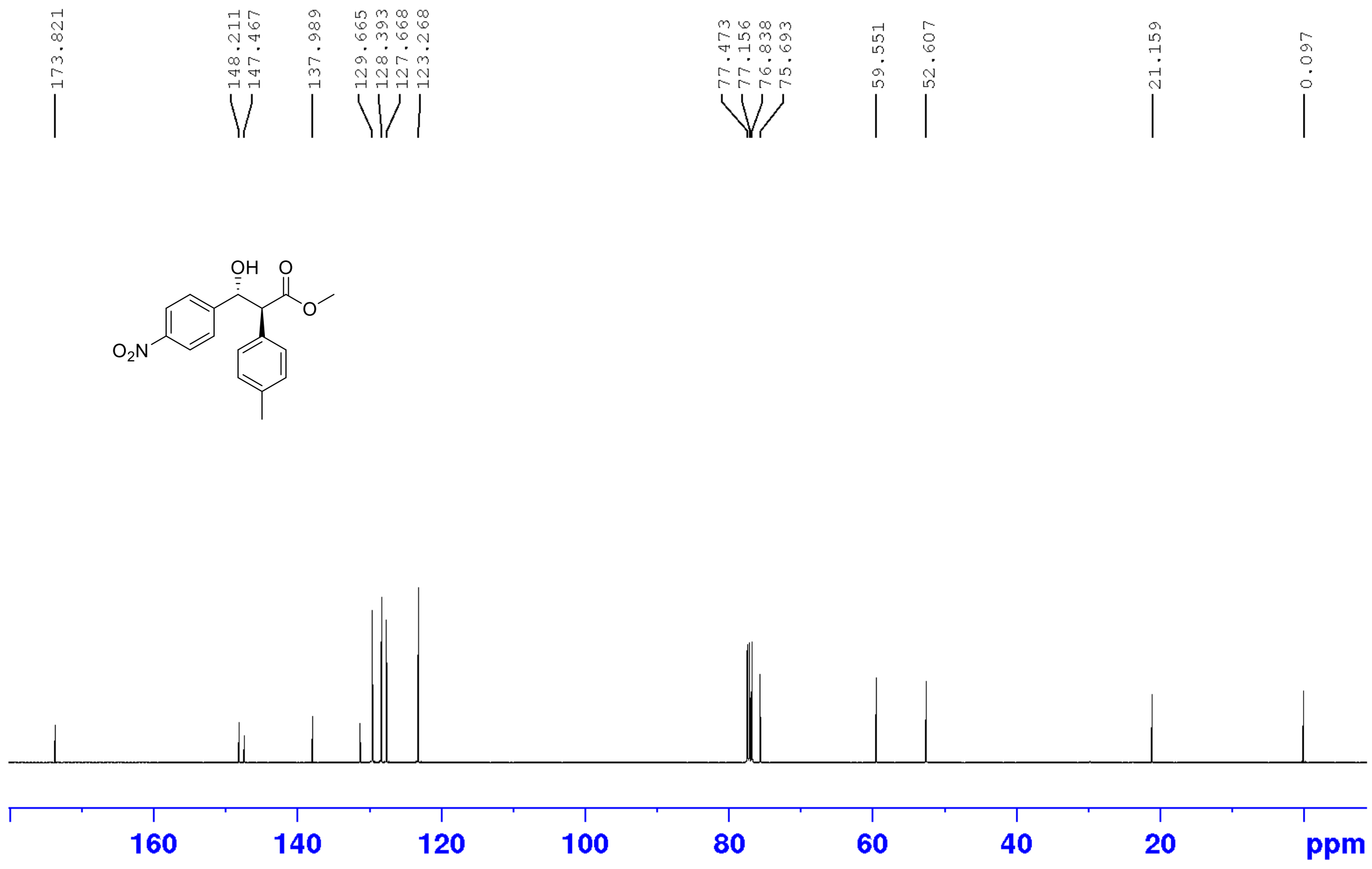

${ }^{13} \mathrm{C}\left\{{ }^{1} \mathrm{H}\right\}$ NMR (100 MHz, $\mathrm{CDCl}_{3}$ ) Spectrum of anti-methyl 3-hydroxy-3-(4-nitrophenyl)-2-p-tolylpropanoate (7e) 

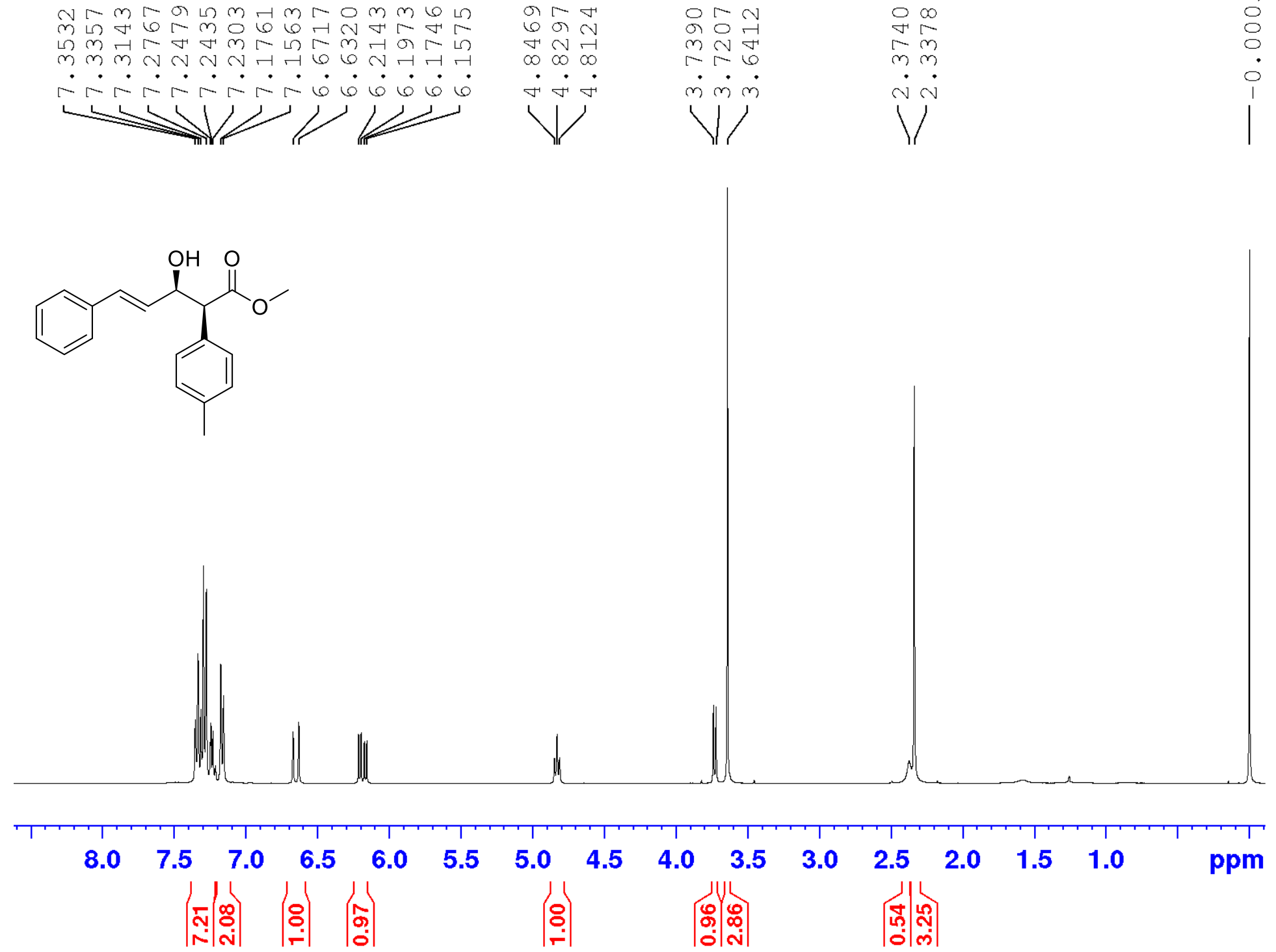

${ }^{1} \mathrm{H}$ NMR (400 MHz, $\mathrm{CDCl}_{3}$ ) Spectrum of syn-(E)-methyl 3-hydroxy-5-phenyl-2-p-tolylpent-4-enoate (7f) 

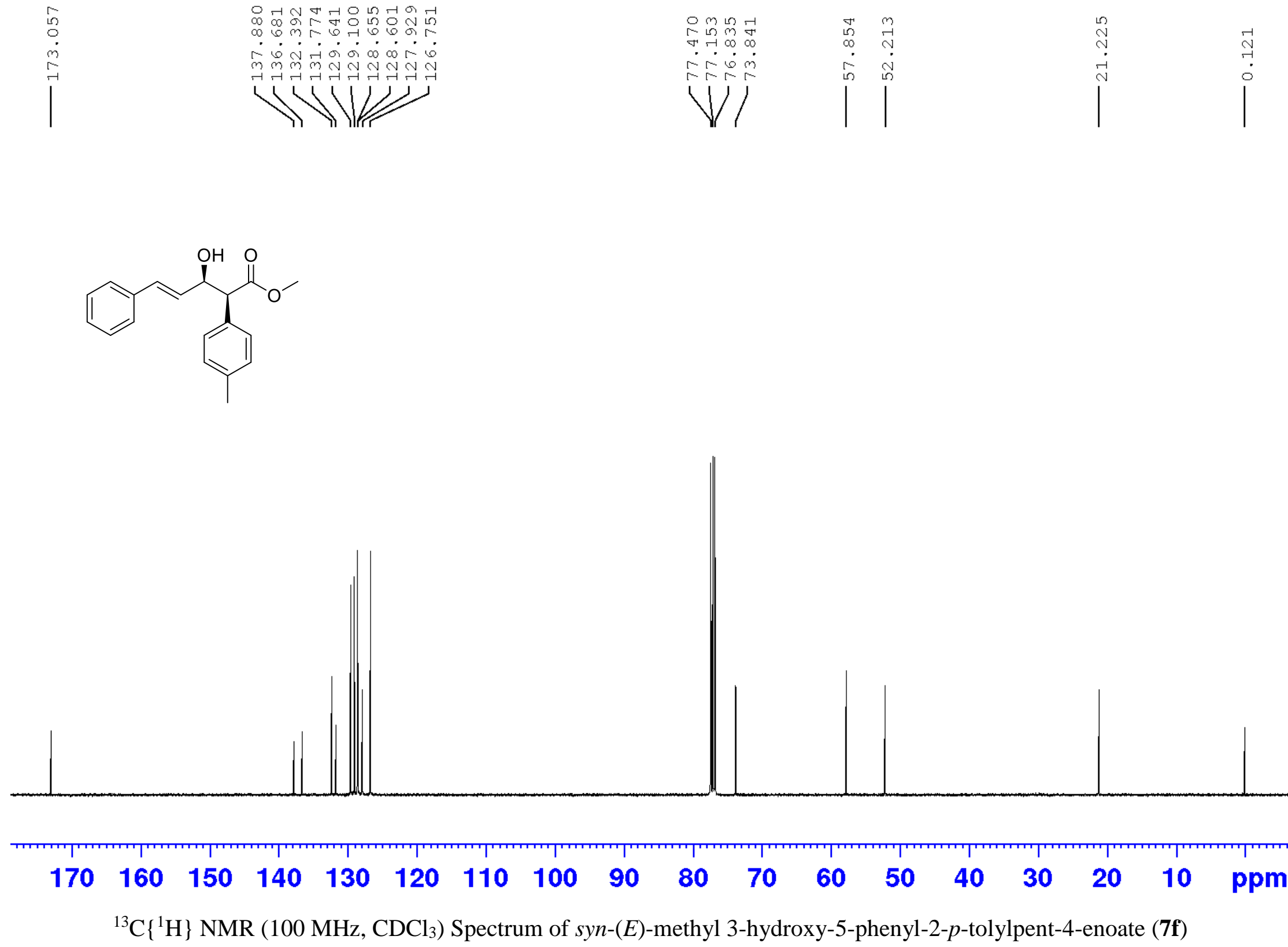


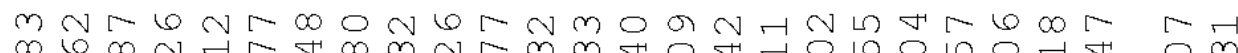

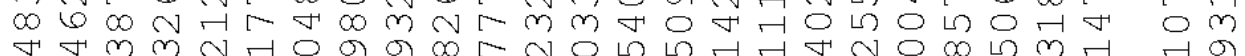
オ NAn $\mathrm{A}$ न

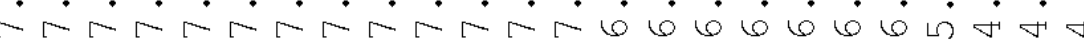
$\longrightarrow$
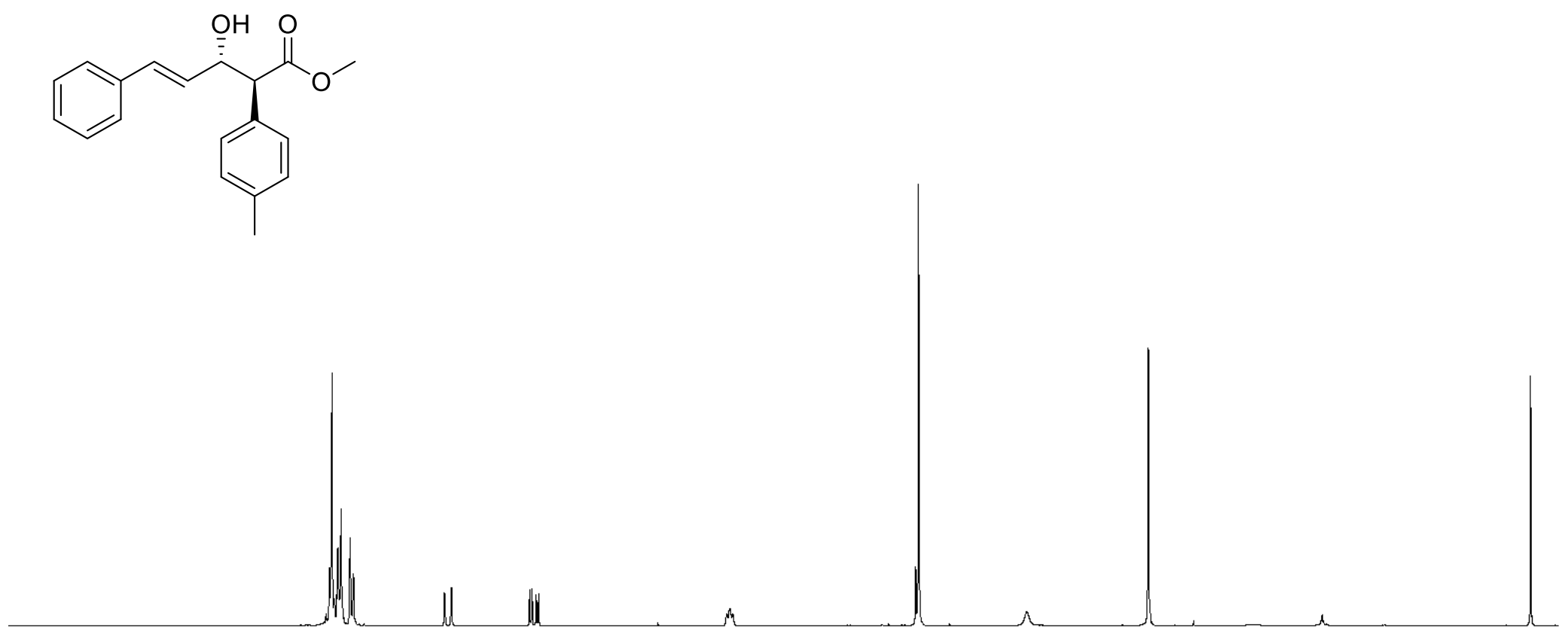

$M$
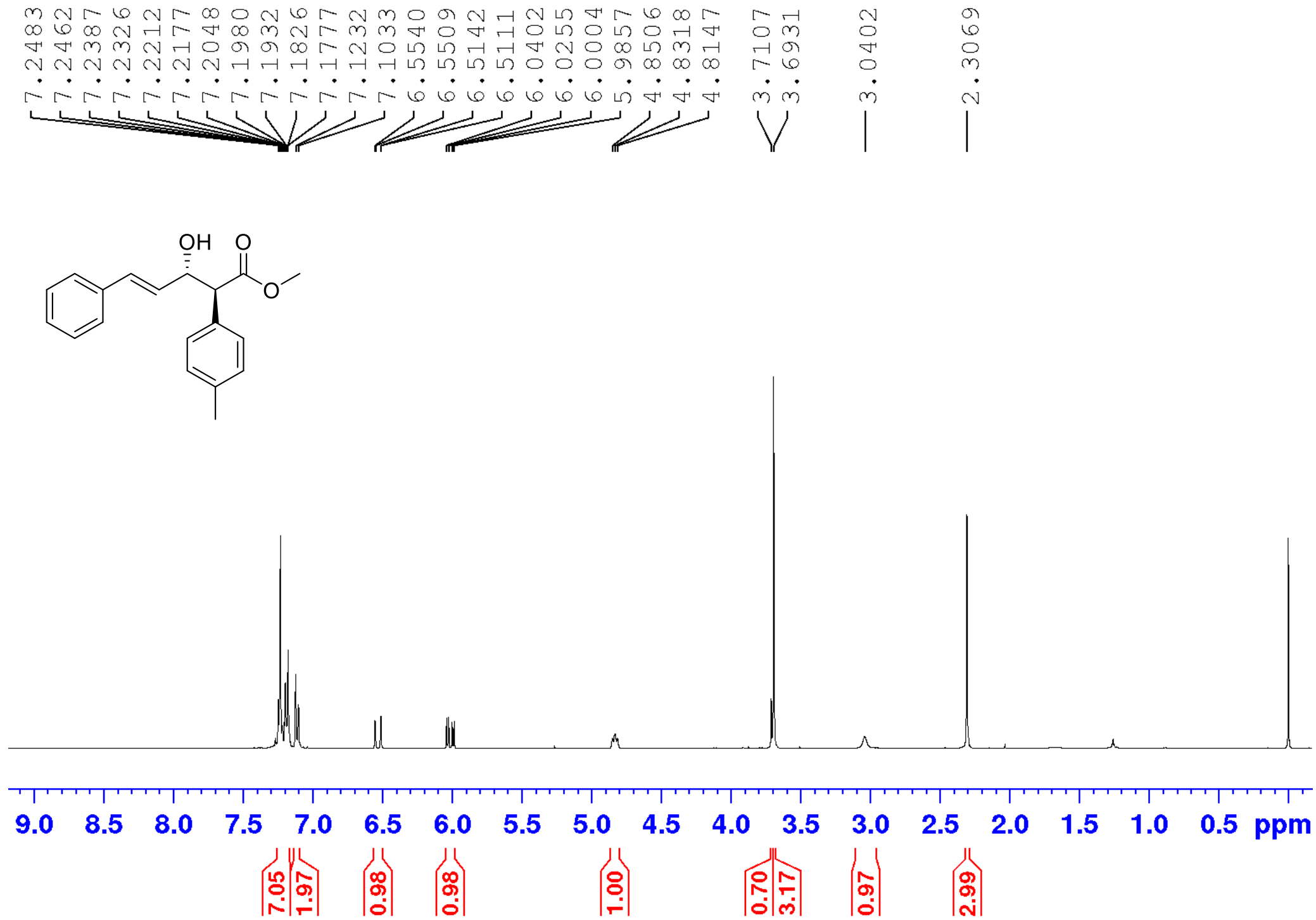

${ }^{1} \mathrm{H}$ NMR (400 MHz, $\mathrm{CDCl}_{3}$ ) Spectrum of anti-(E)-methyl 3-hydroxy-5-phenyl-2-p-tolylpent-4-enoate (7f) 

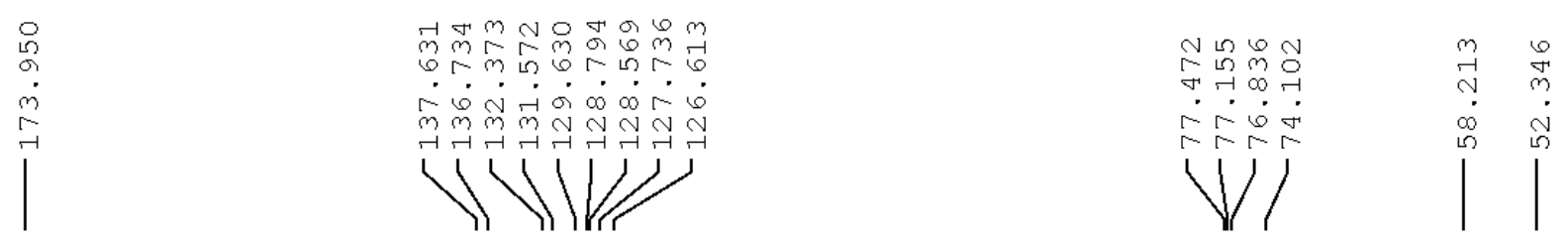

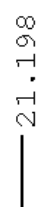

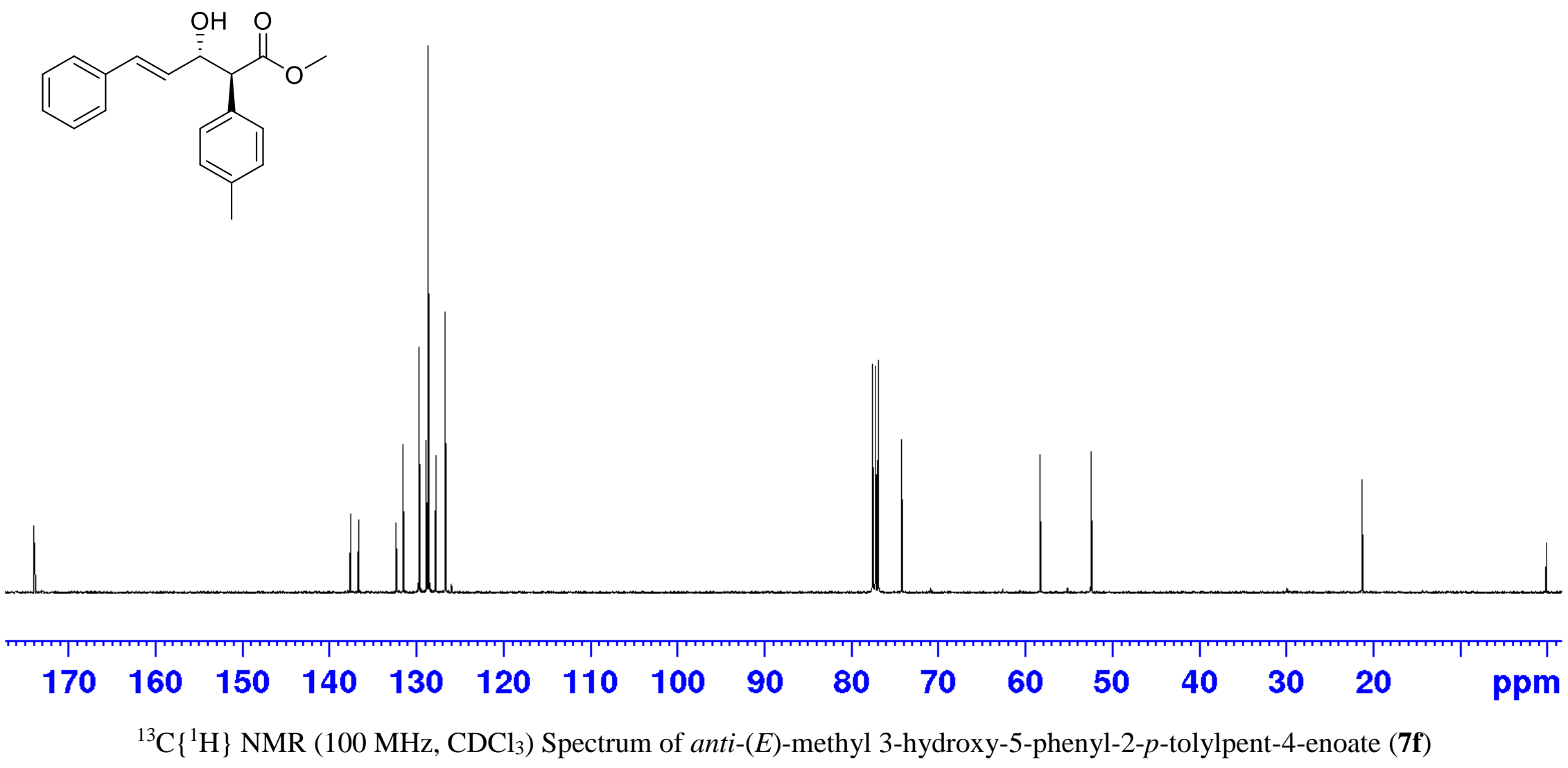



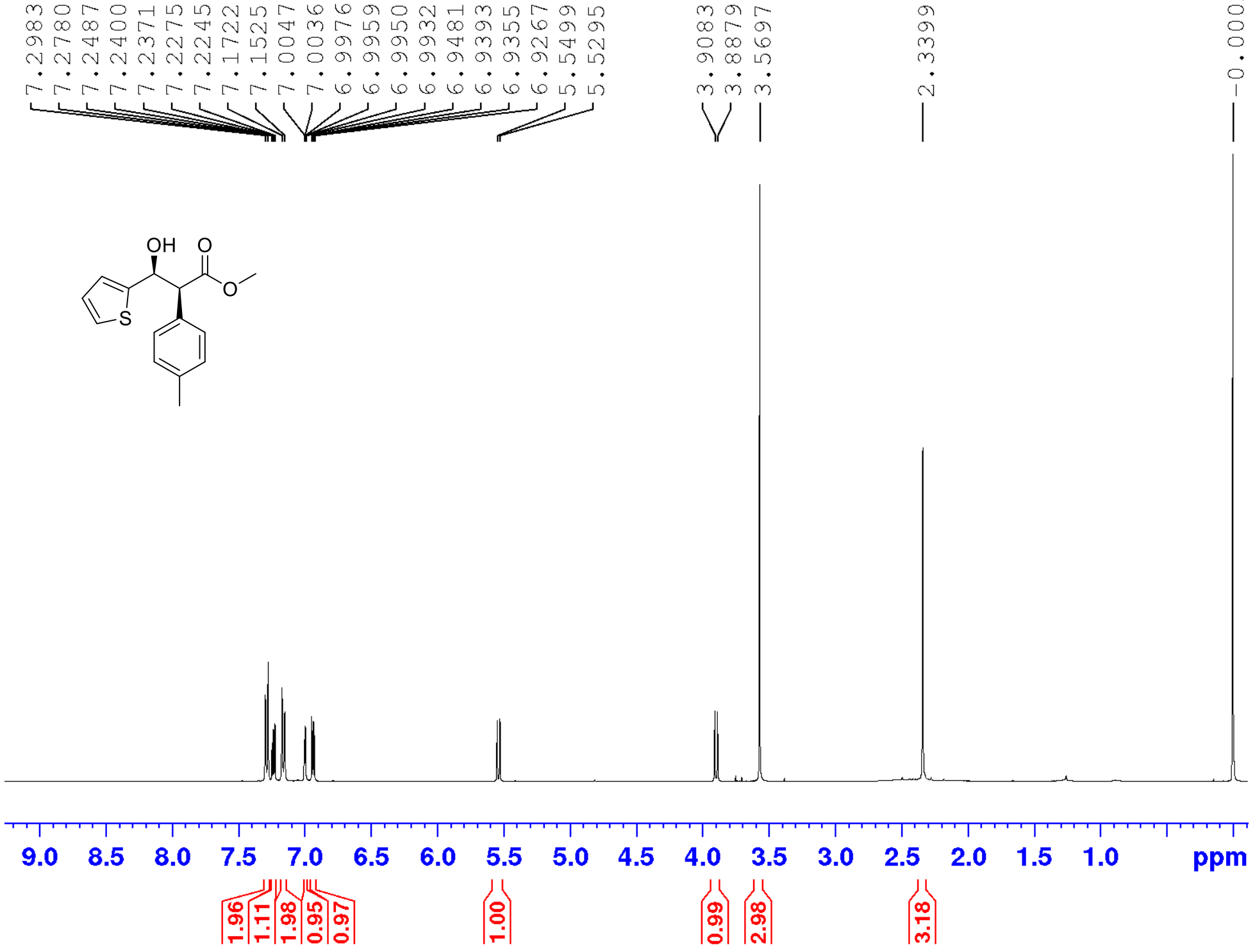

${ }^{1} \mathrm{H}$ NMR (400 MHz, $\mathrm{CDCl}_{3}$ ) Spectrum of syn-methyl 3-hydroxy-3-(thiophen-2-yl)-2-p-tolylpropanoate (7g) 

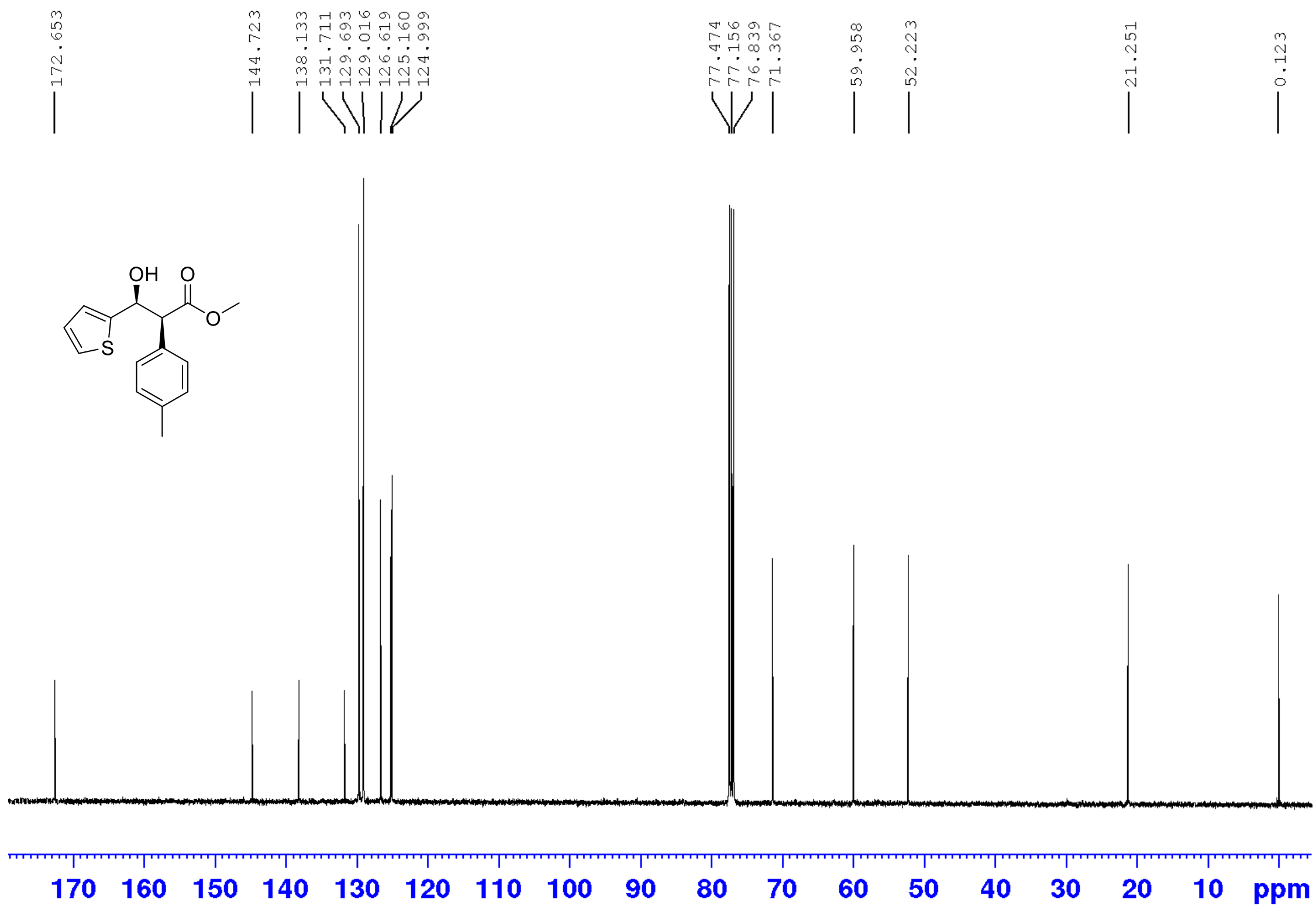

${ }^{13} \mathrm{C}\left\{{ }^{1} \mathrm{H}\right\} \mathrm{NMR}\left(100 \mathrm{MHz}, \mathrm{CDCl}_{3}\right)$ Spectrum of syn-methyl 3-hydroxy-3-(thiophen-2-yl)-2-p-tolylpropanoate (7g) 
न $m$ M 가.

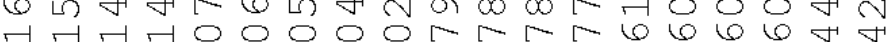

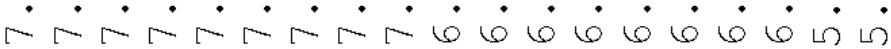
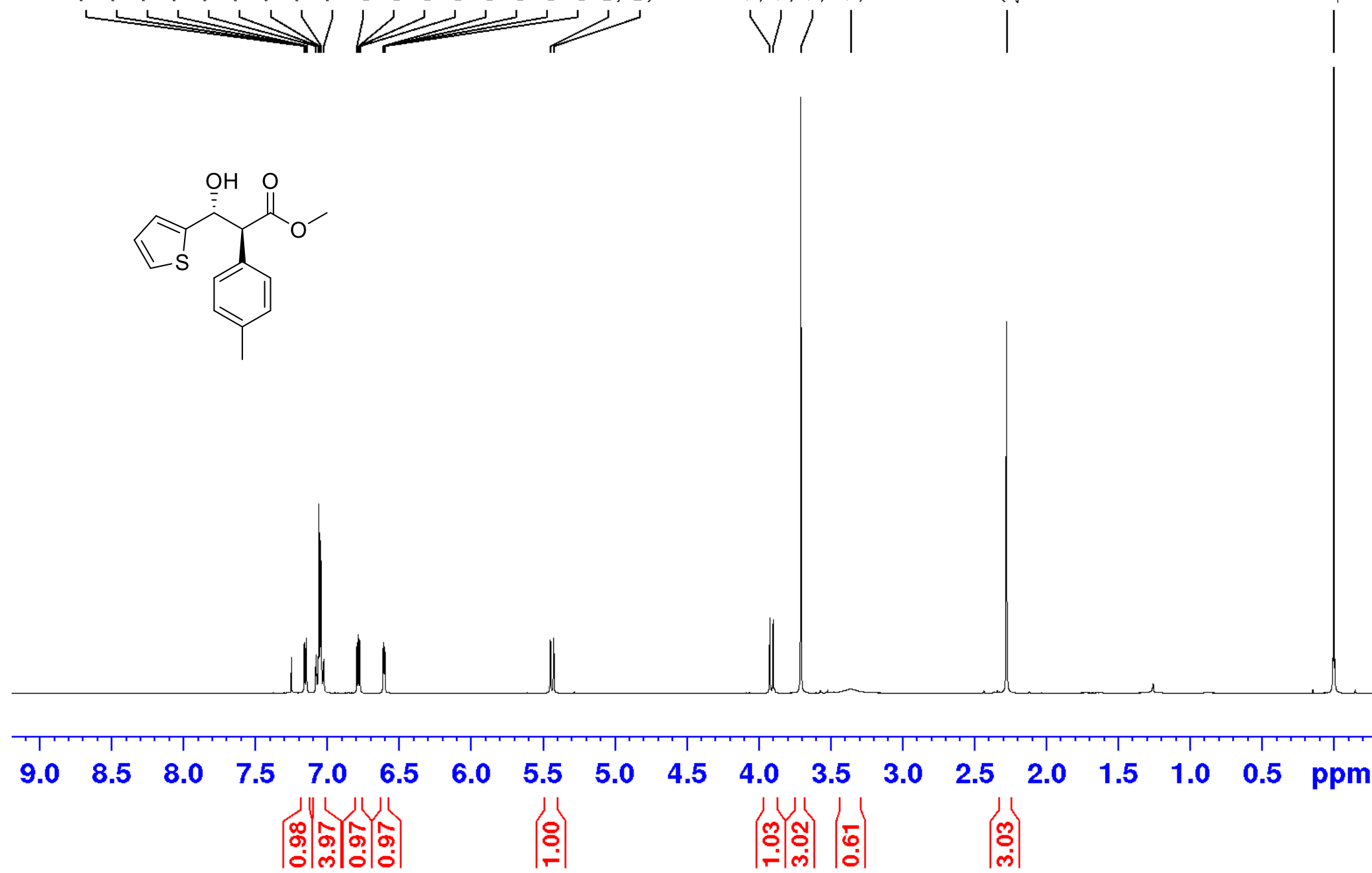

${ }^{1} \mathrm{H}$ NMR (400 MHz, $\mathrm{CDCl}_{3}$ ) Spectrum of anti-methyl 3-hydroxy-3-(thiophen-2-yl)-2-p-tolylpropanoate (7g) 


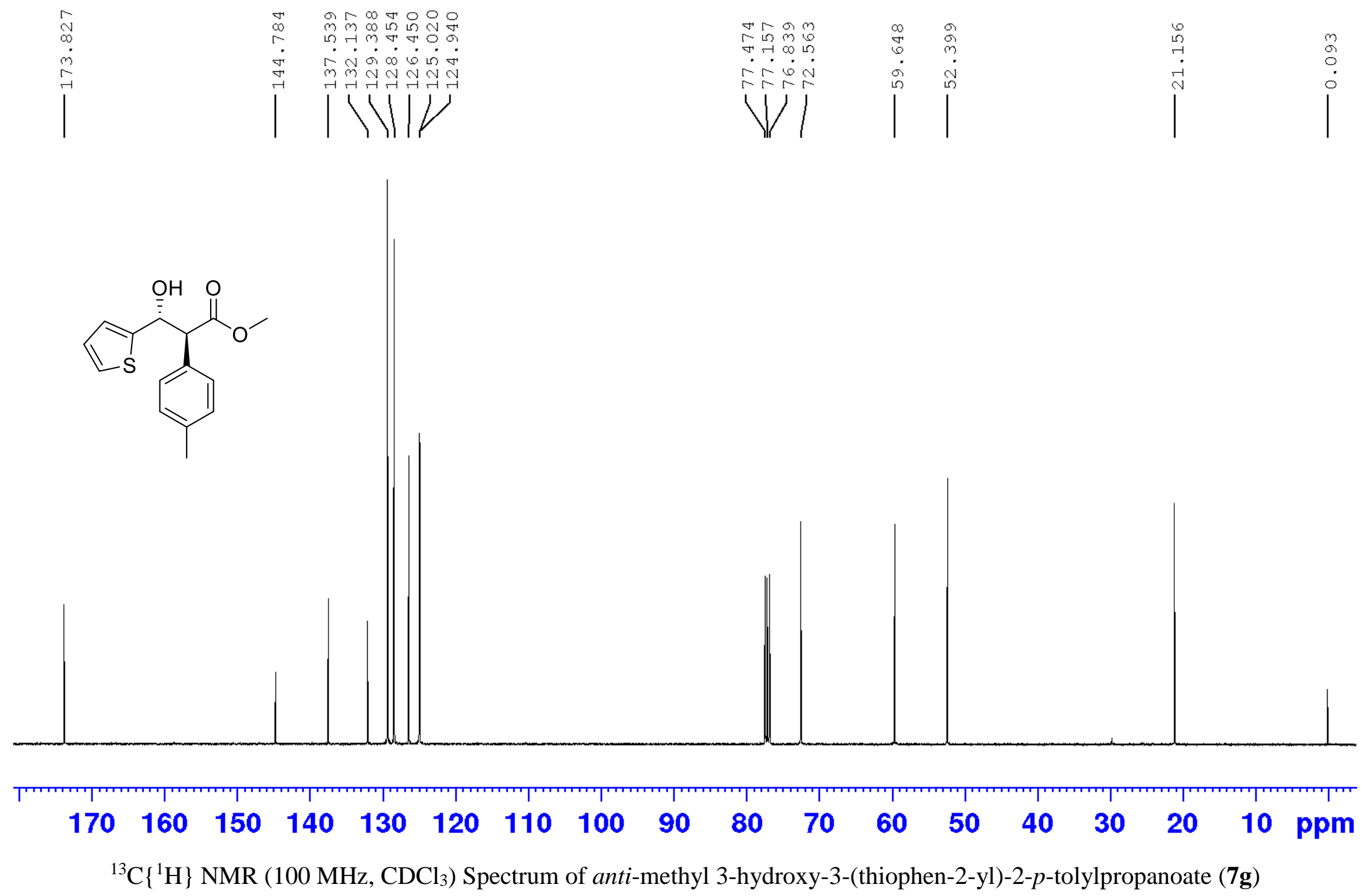




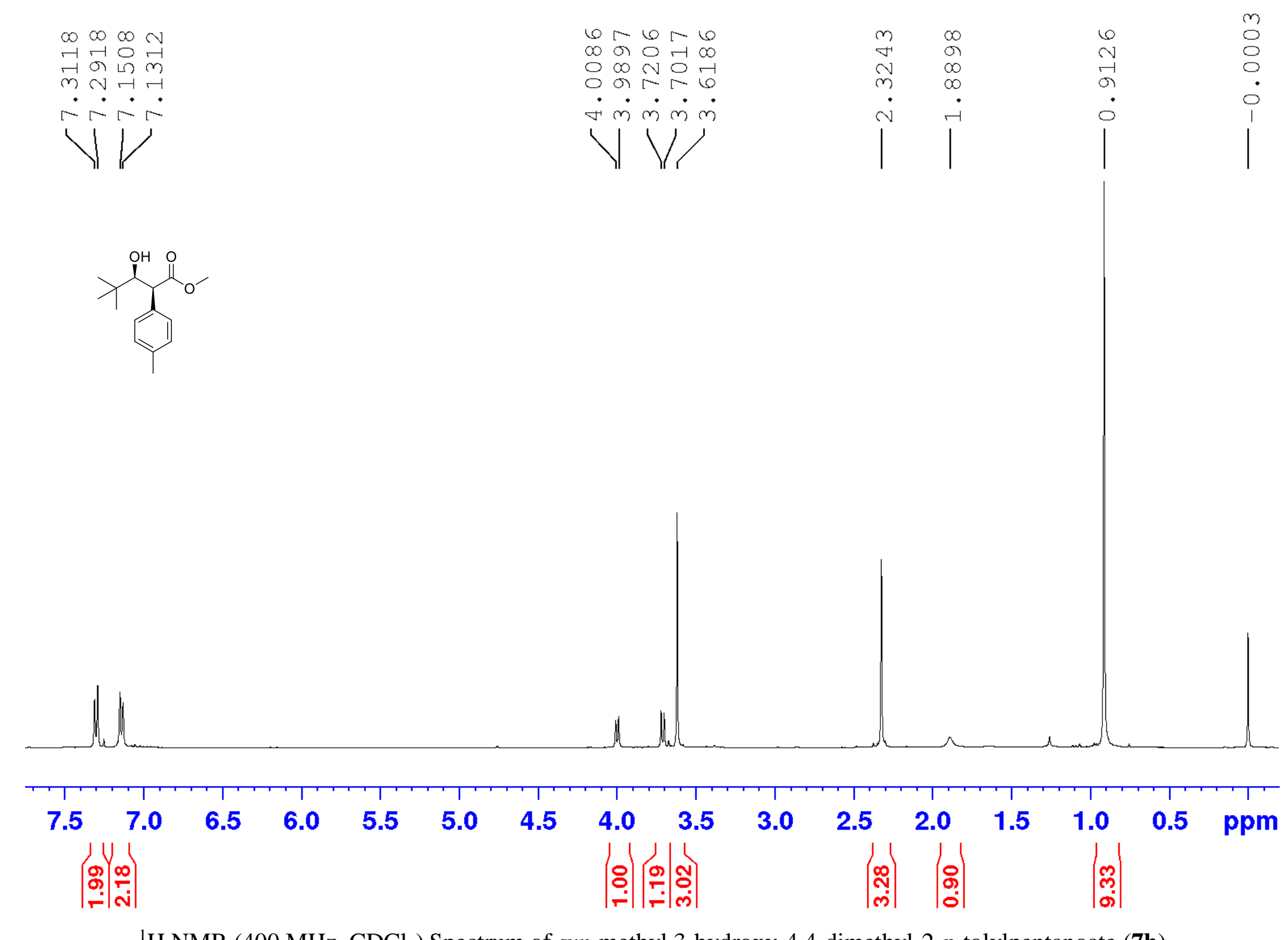

${ }^{1} \mathrm{H}$ NMR (400 MHz, $\mathrm{CDCl}_{3}$ ) Spectrum of syn-methyl 3-hydroxy-4,4-dimethyl-2-p-tolylpentanoate (7h) 

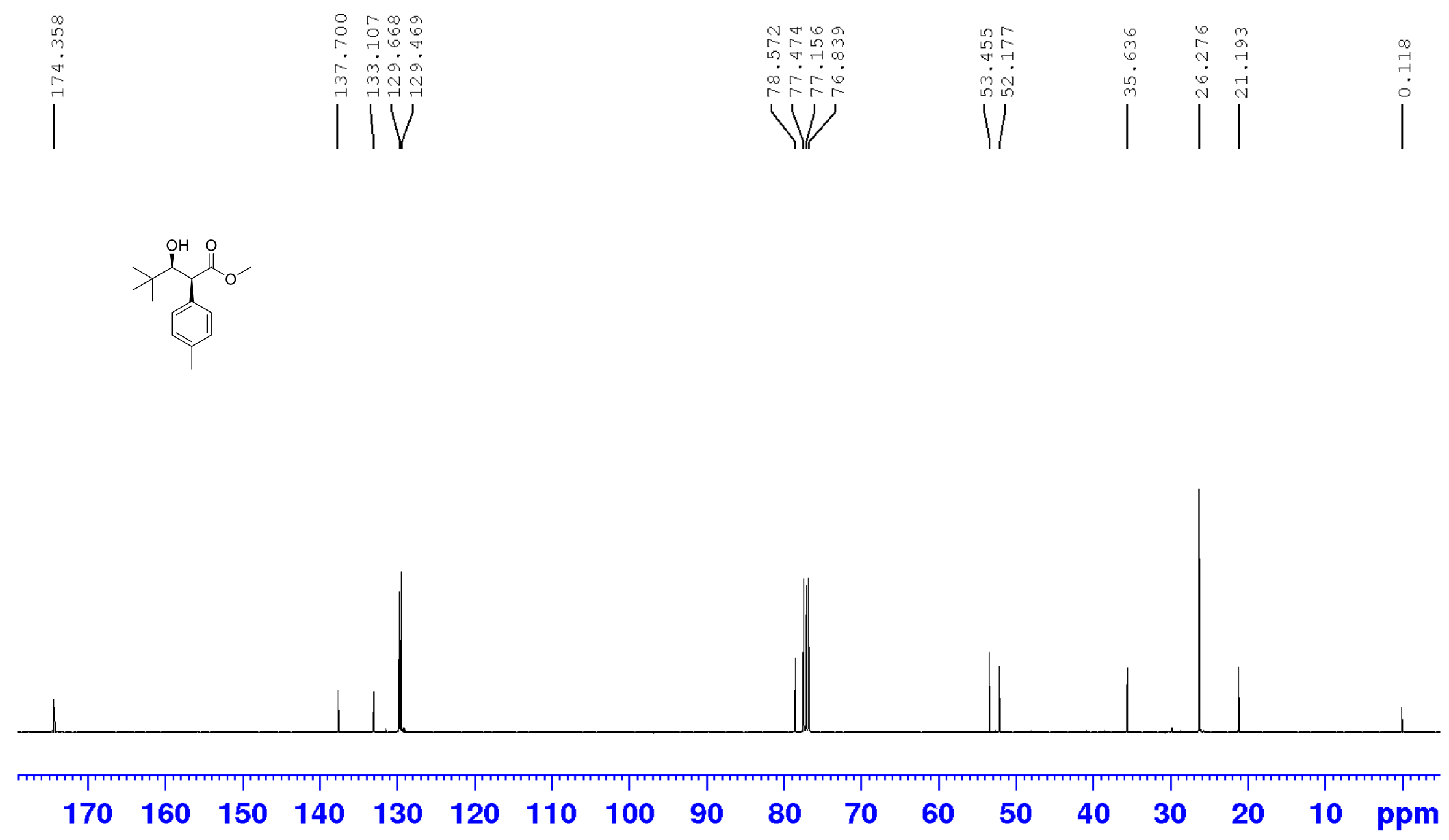

${ }^{13} \mathrm{C}\left\{{ }^{1} \mathrm{H}\right\}$ NMR (100 MHz, $\left.\mathrm{CDCl}_{3}\right)$ Spectrum of syn-methyl 3-hydroxy-4,4-dimethyl-2-p-tolylpentanoate (7h) 

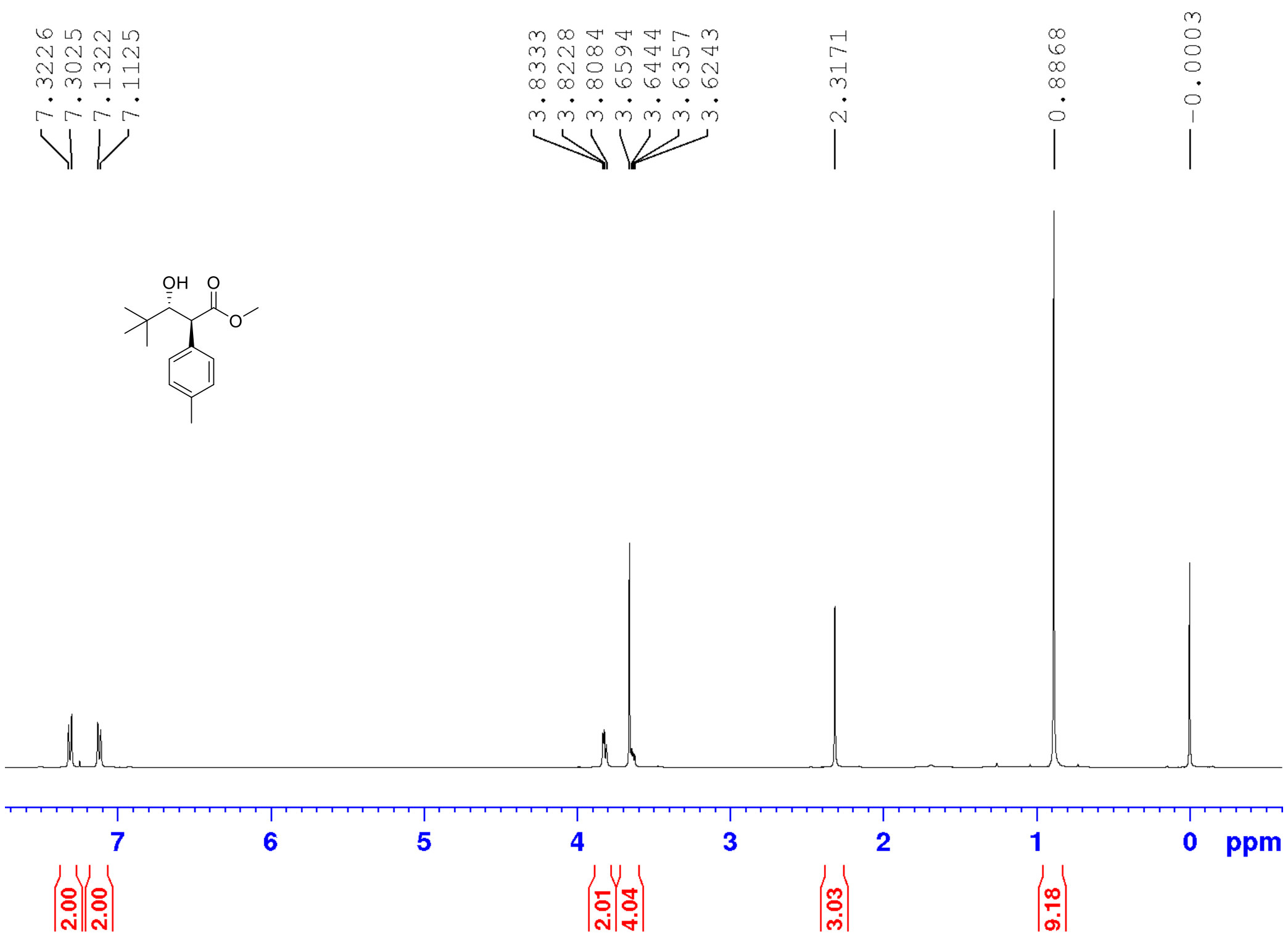

${ }^{1} \mathrm{H}$ NMR (400 MHz, $\mathrm{CDCl}_{3}$ ) Spectrum of anti-methyl 3-hydroxy-4,4-dimethyl-2-p-tolylpentanoate (7h) 


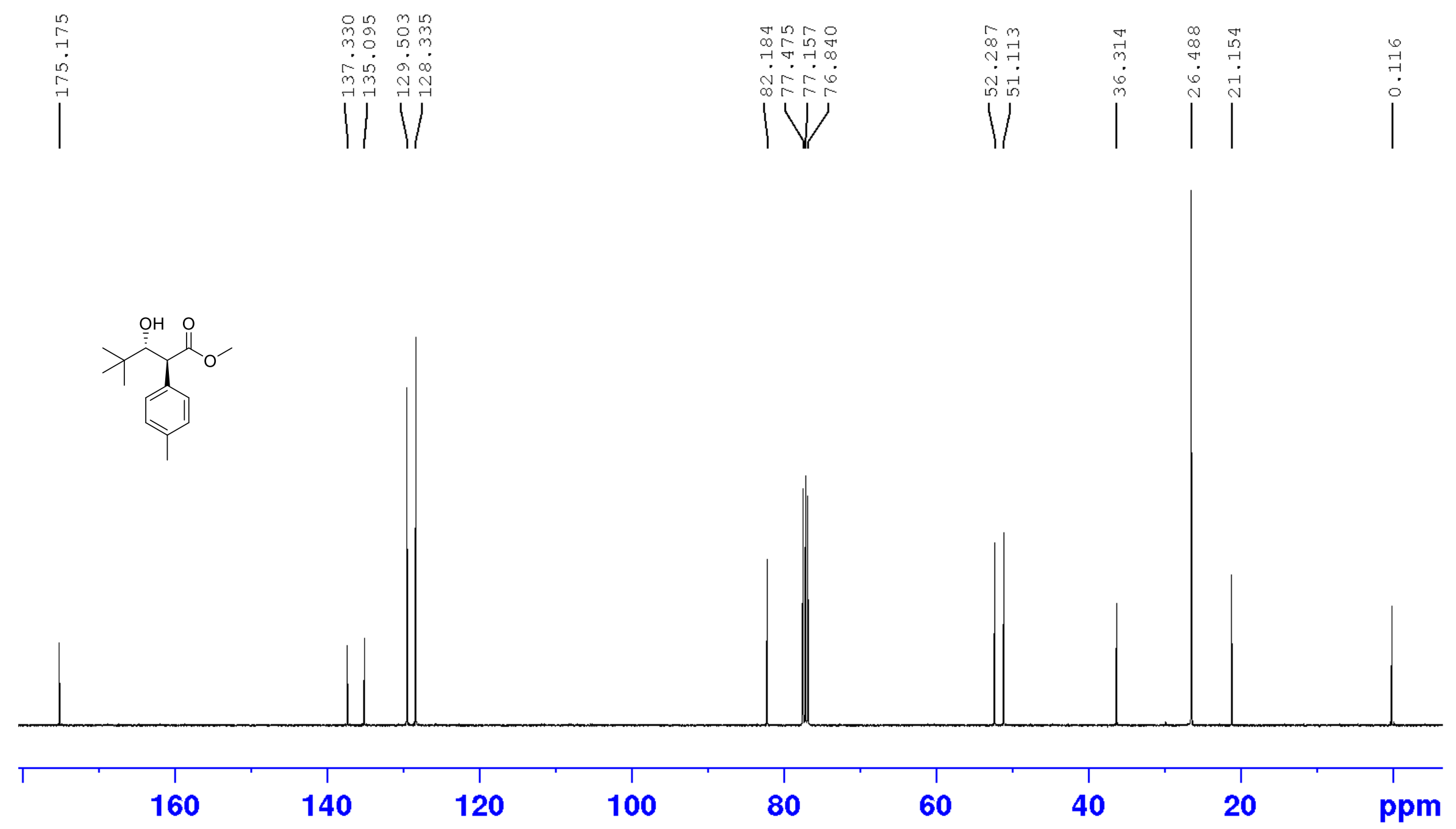

${ }^{13} \mathrm{C}\left\{{ }^{1} \mathrm{H}\right\}$ NMR $\left(100 \mathrm{MHz}, \mathrm{CDCl}_{3}\right)$ Spectrum of anti-methyl 3-hydroxy-4,4-dimethyl-2-p-tolylpentanoate (7h) 

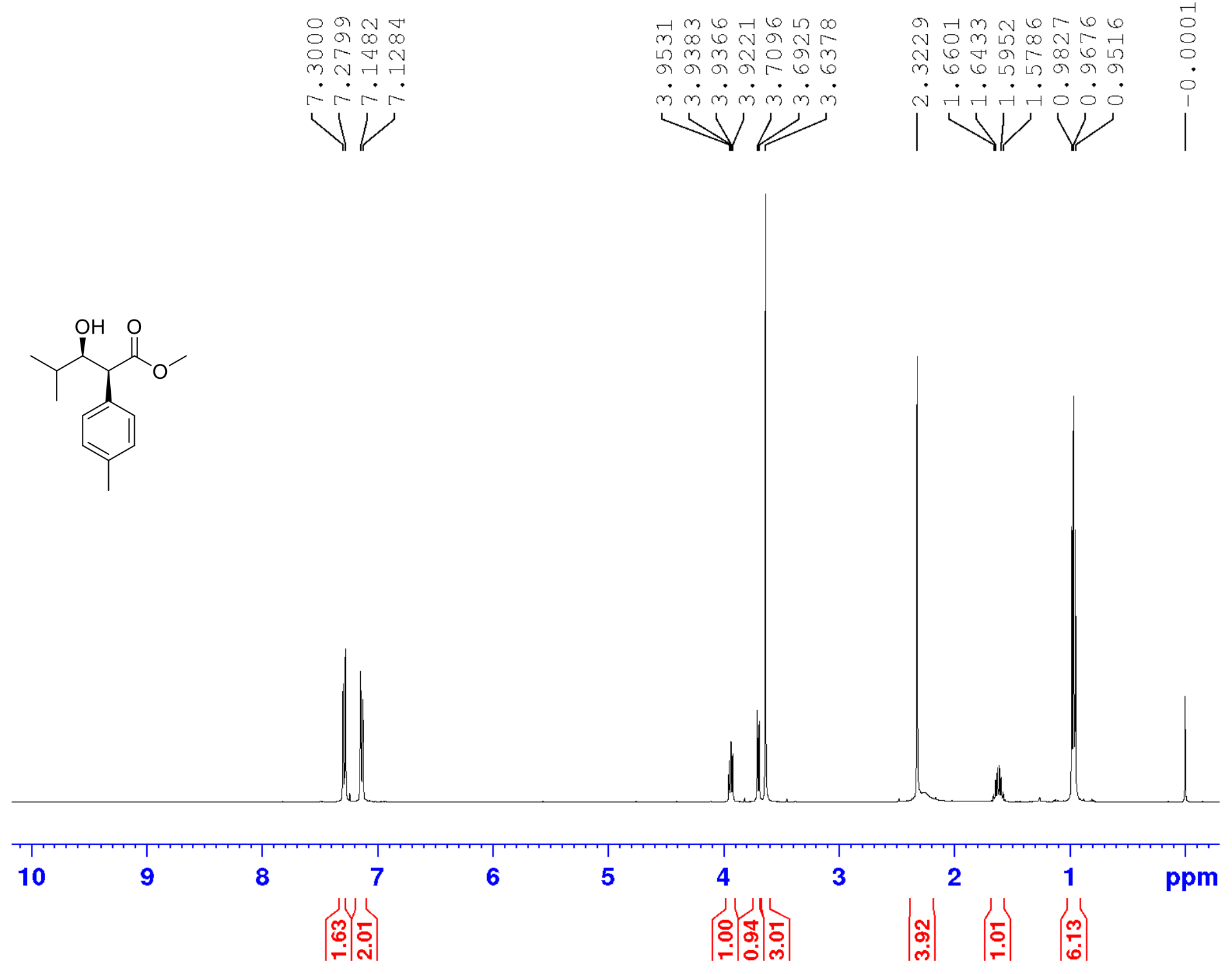

${ }^{1} \mathrm{H}$ NMR (400 MHz, $\mathrm{CDCl}_{3}$ ) Spectrum of syn-methyl 3-hydroxy-4-methyl-2-p-tolylpentanoate (7i) 


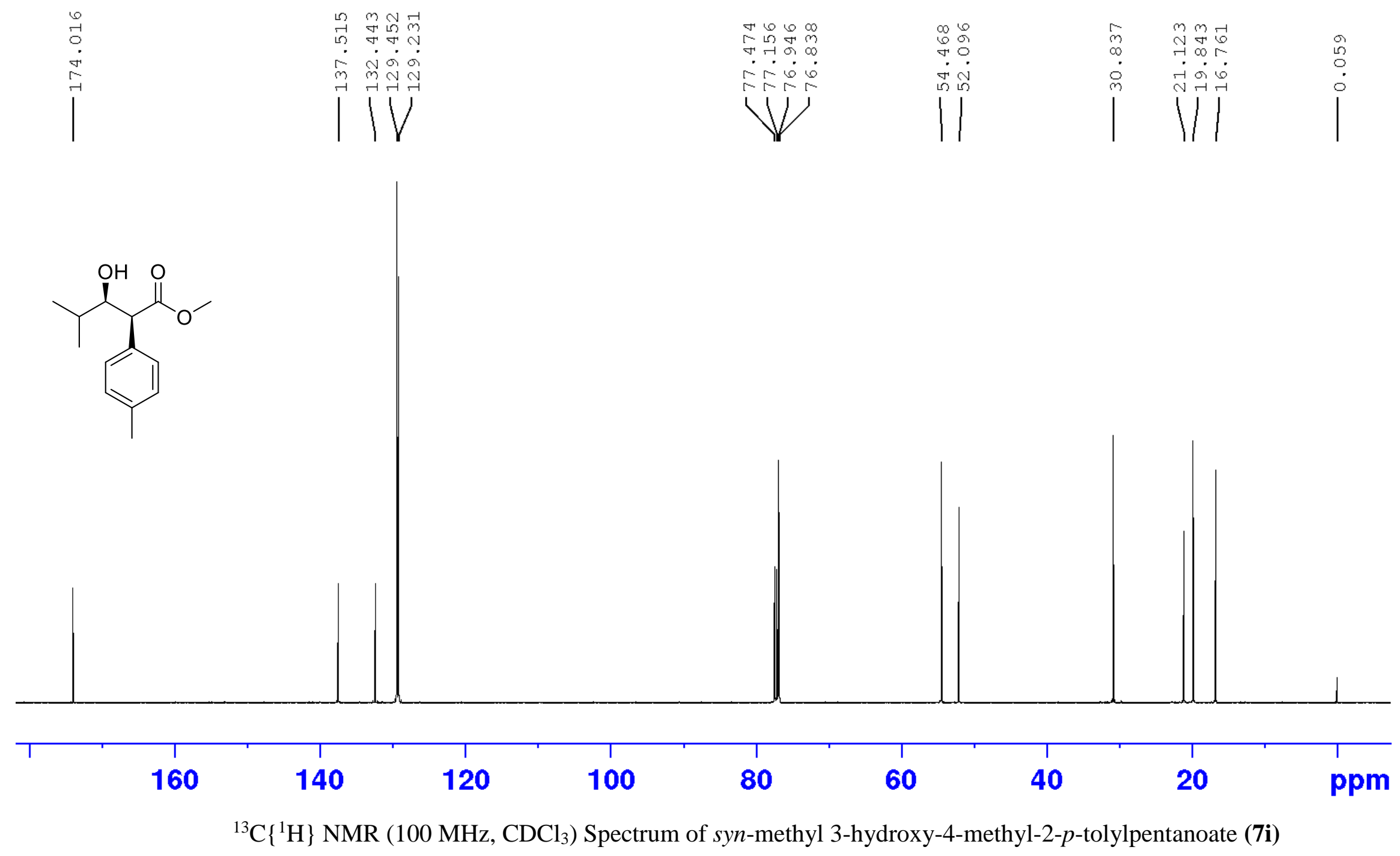



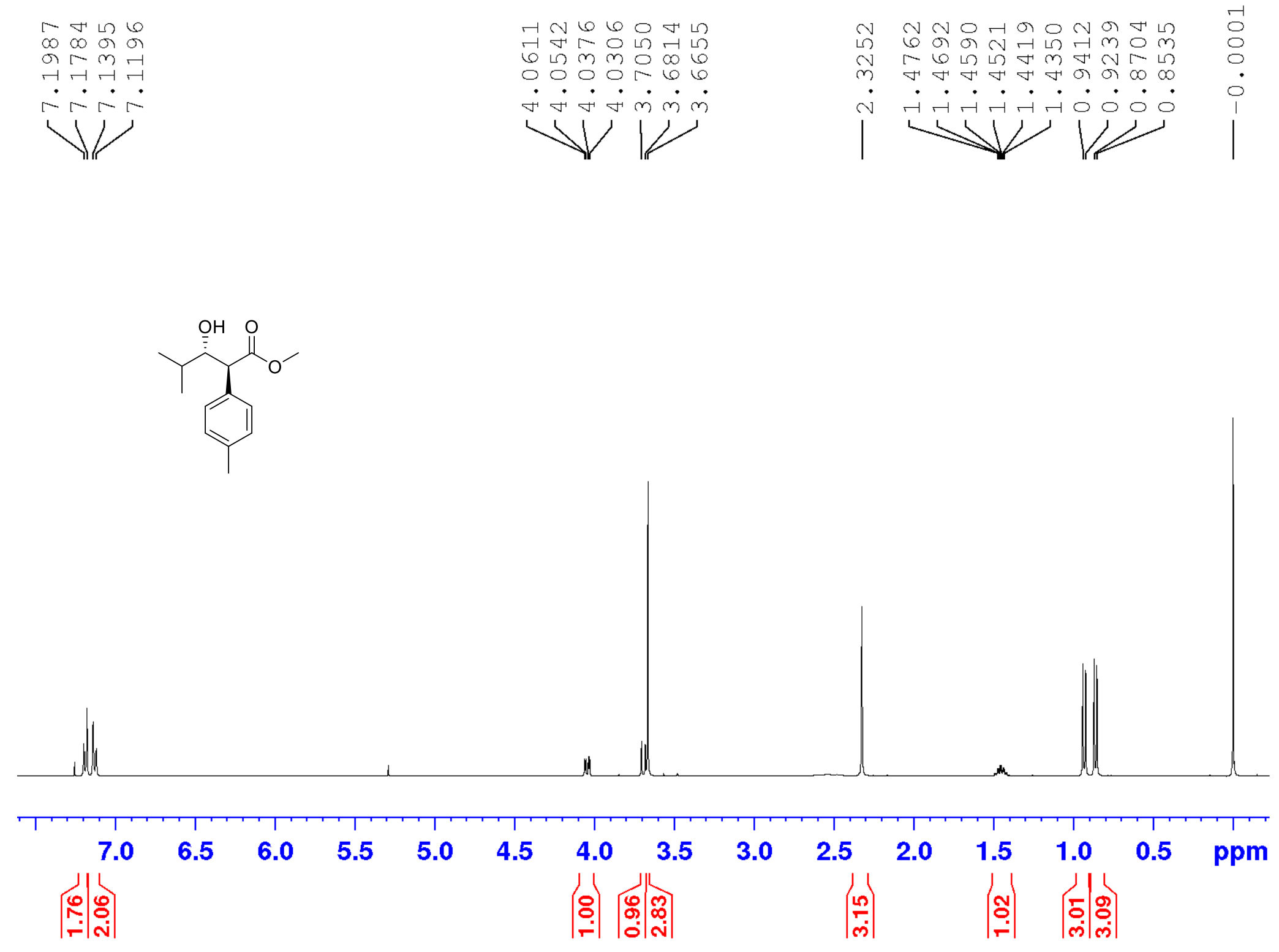

${ }^{1} \mathrm{H}$ NMR (400 MHz, $\mathrm{CDCl}_{3}$ ) Spectrum of anti-methyl 3-hydroxy-4-methyl-2-p-tolylpentanoate (7i) 

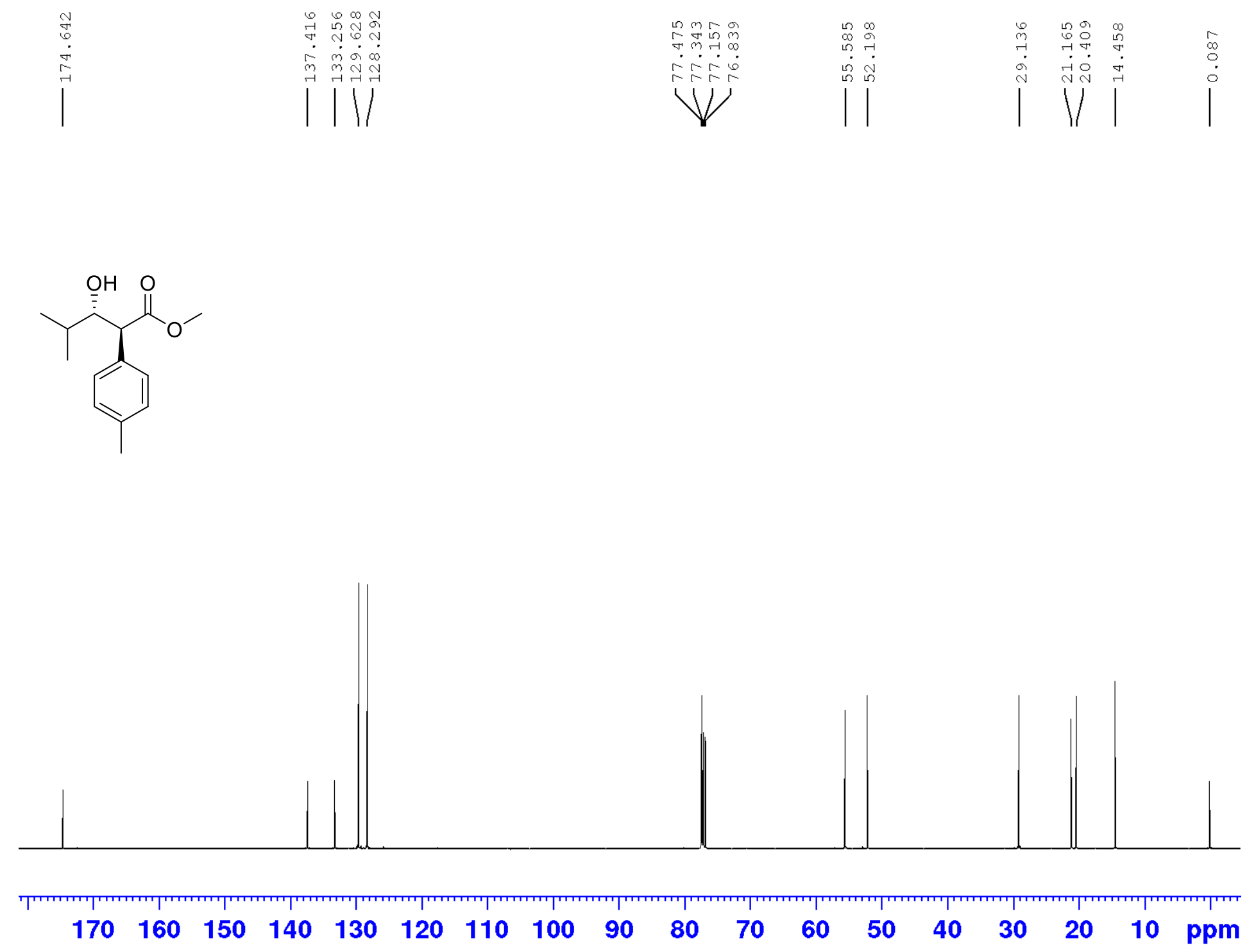

${ }^{13} \mathrm{C}\left\{{ }^{1} \mathrm{H}\right\}$ NMR (100 MHz, $\mathrm{CDCl}_{3}$ ) Spectrum of anti-methyl 3-hydroxy-4-methyl-2-p-tolylpentanoate (7i) 


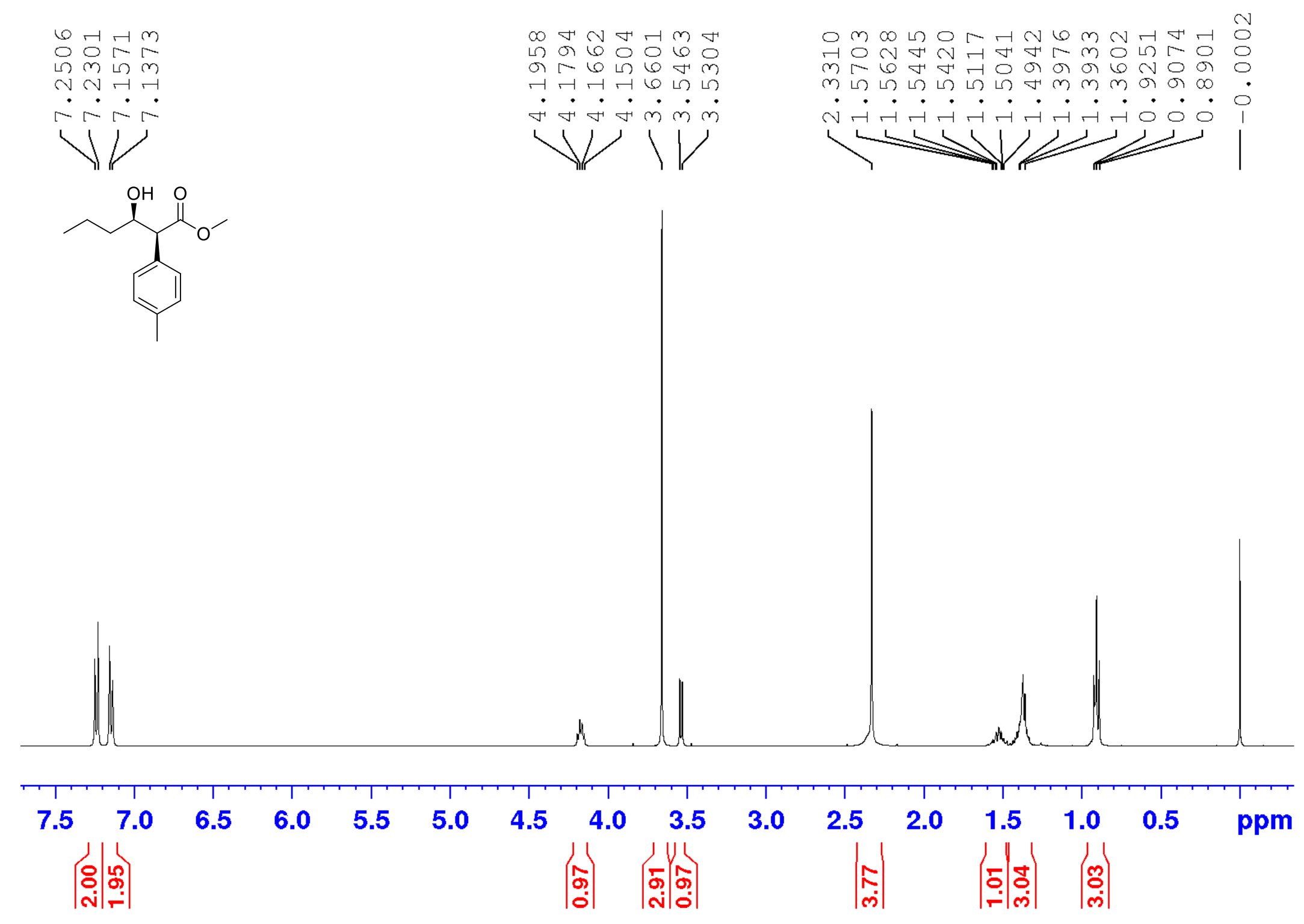

${ }^{1} \mathrm{H}$ NMR (400 MHz, $\mathrm{CDCl}_{3}$ ) Spectrum of syn-methyl 3-hydroxy-2-p-tolylhexanoate (7j) 

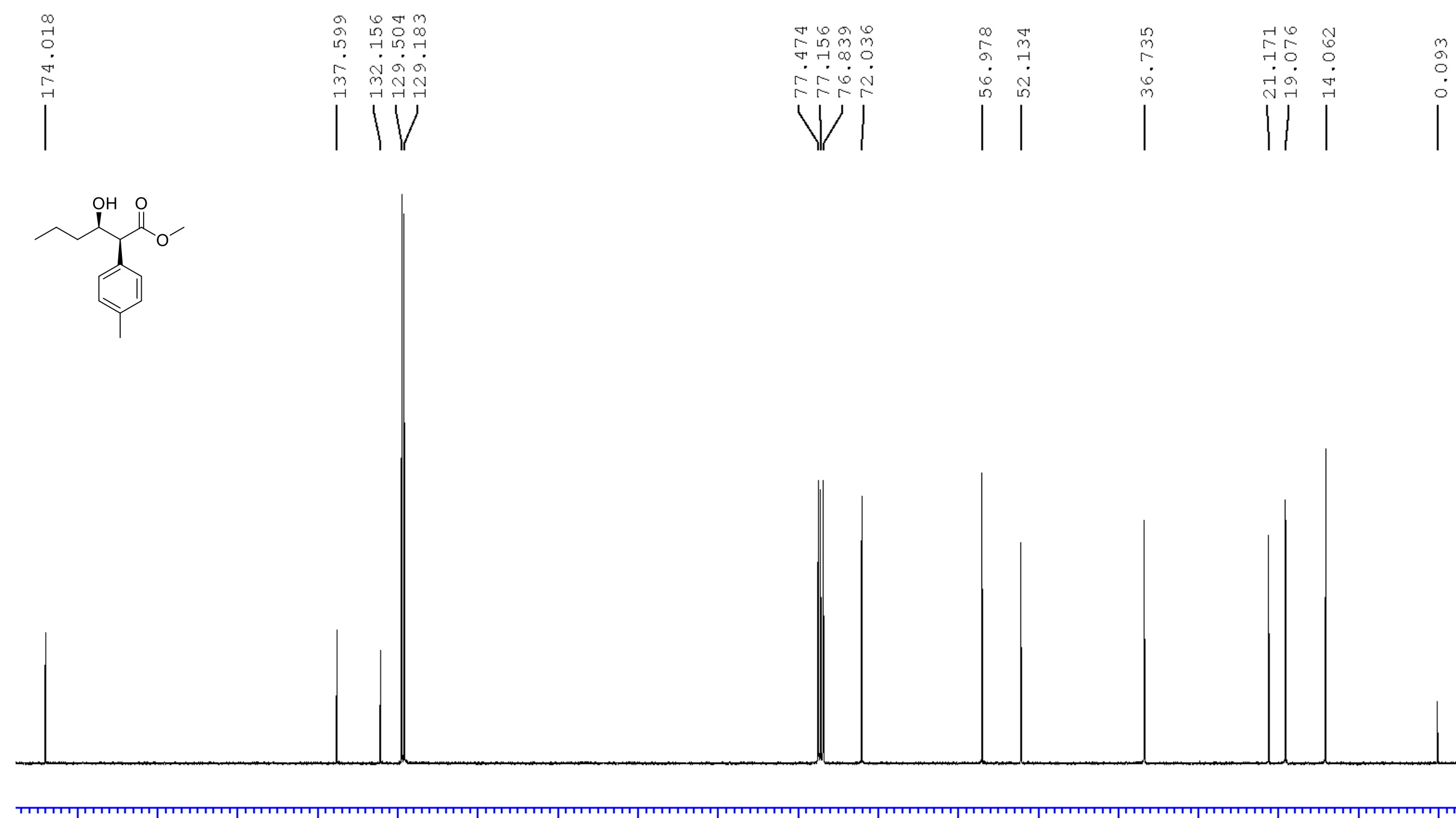

170160 $\begin{array}{lll}150 & 140 & 130\end{array}$

${ }^{13} \mathrm{C}\left\{{ }^{1} \mathrm{H}\right\} \mathrm{NMR}\left(100 \mathrm{MHz}, \mathrm{CDCl}_{3}\right)$ Spectrum of syn-methyl 3-hydroxy-2-p-tolylhexanoate (7j) 
m

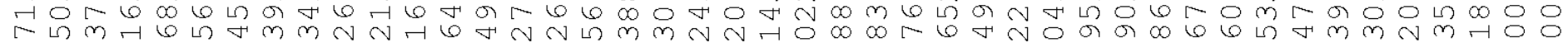
H.

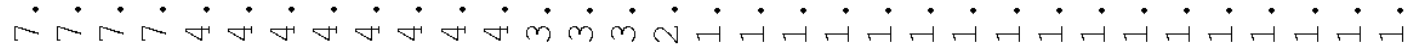

1/ 1

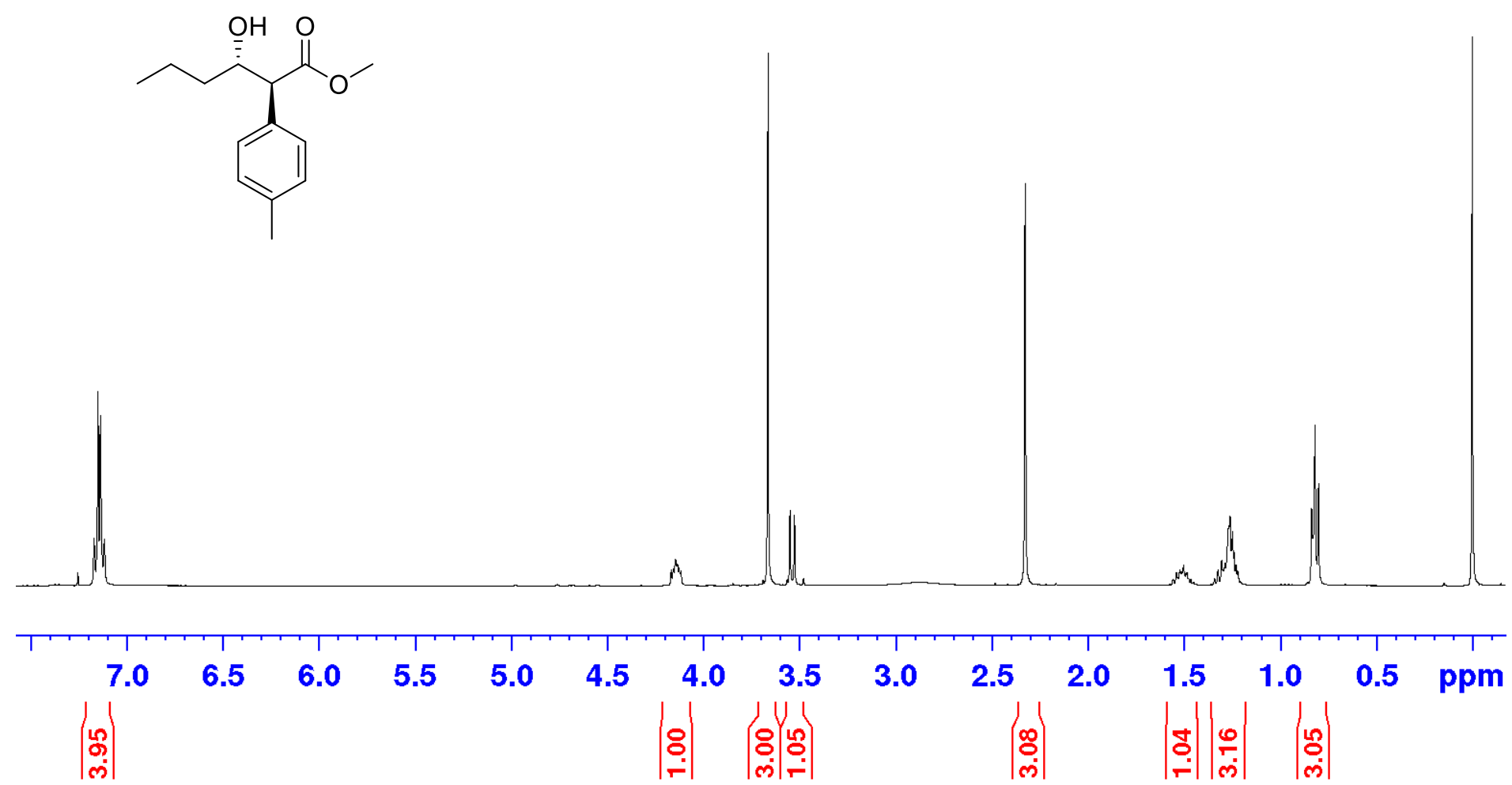

${ }^{1} \mathrm{H} \mathrm{NMR}\left(400 \mathrm{MHz}, \mathrm{CDCl}_{3}\right)$ Spectrum of anti-methyl 3-hydroxy-2-p-tolylhexanoate (7j) 

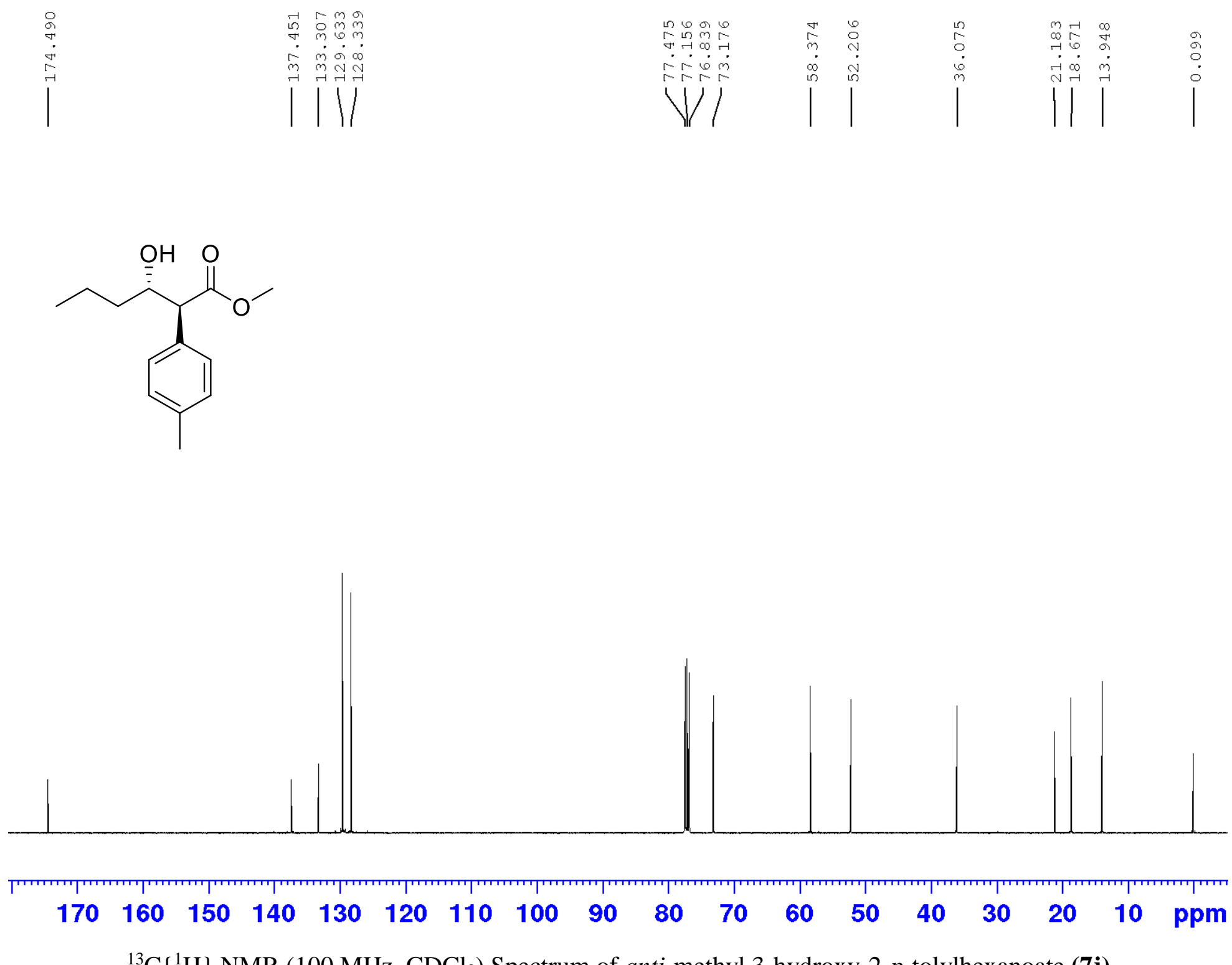

${ }^{13} \mathrm{C}\left\{{ }^{1} \mathrm{H}\right\}$ NMR (100 MHz, $\left.\mathrm{CDCl}_{3}\right)$ Spectrum of anti-methyl 3-hydroxy-2-p-tolylhexanoate (7j) 
అ

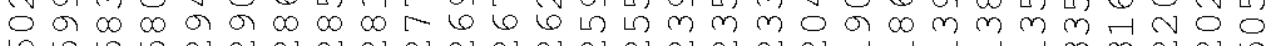

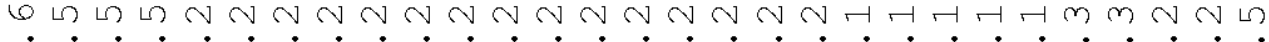

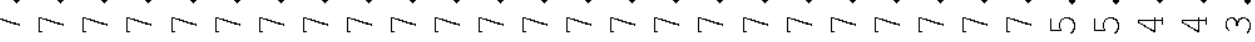

$=b=0=0=0$

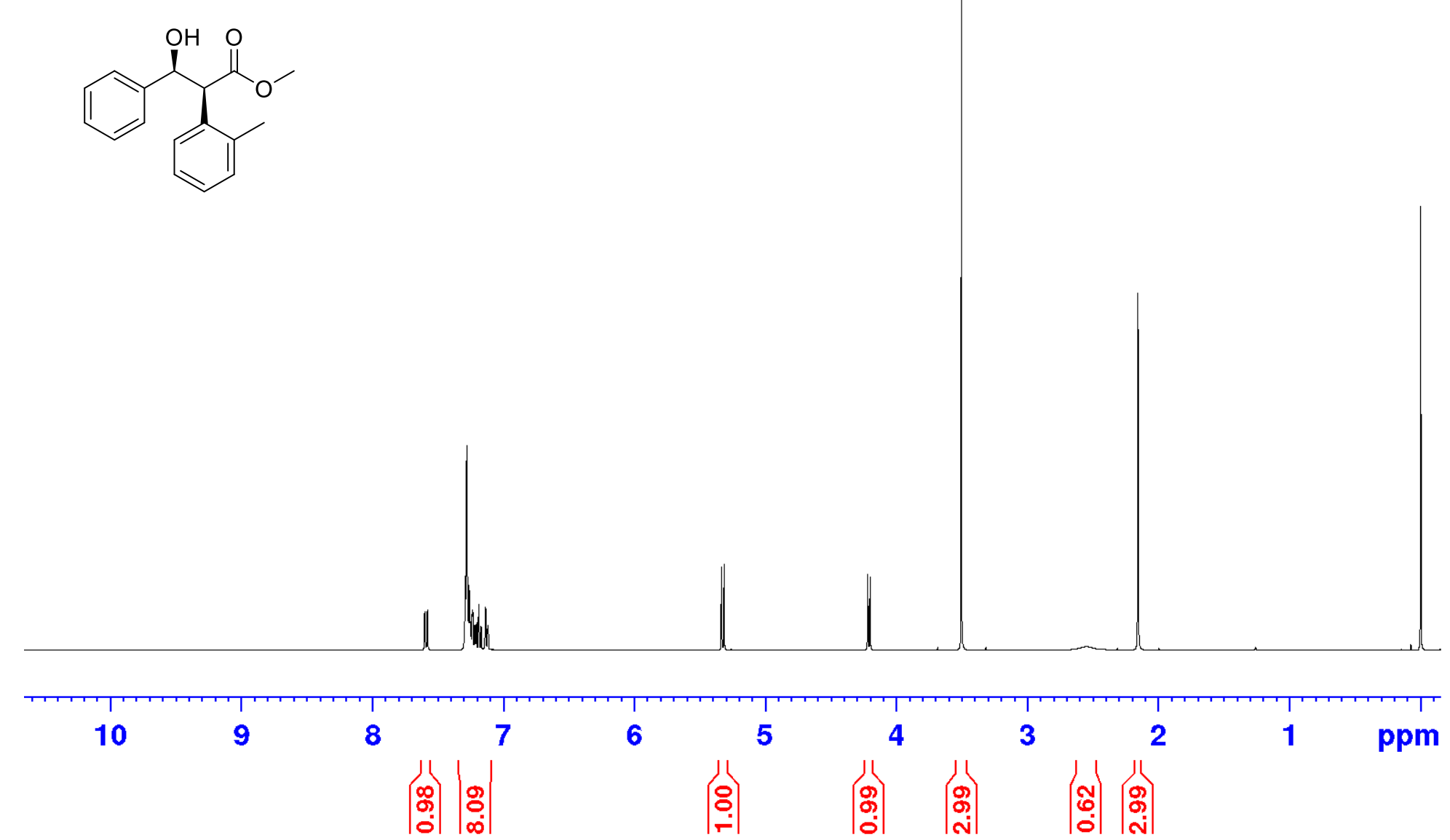

${ }^{1} \mathrm{H}$ NMR (400 MHz, $\mathrm{CDCl}_{3}$ ) Spectrum of syn-methyl 3-hydroxy-3-phenyl-2-o-tolylpropanoate (10a) 

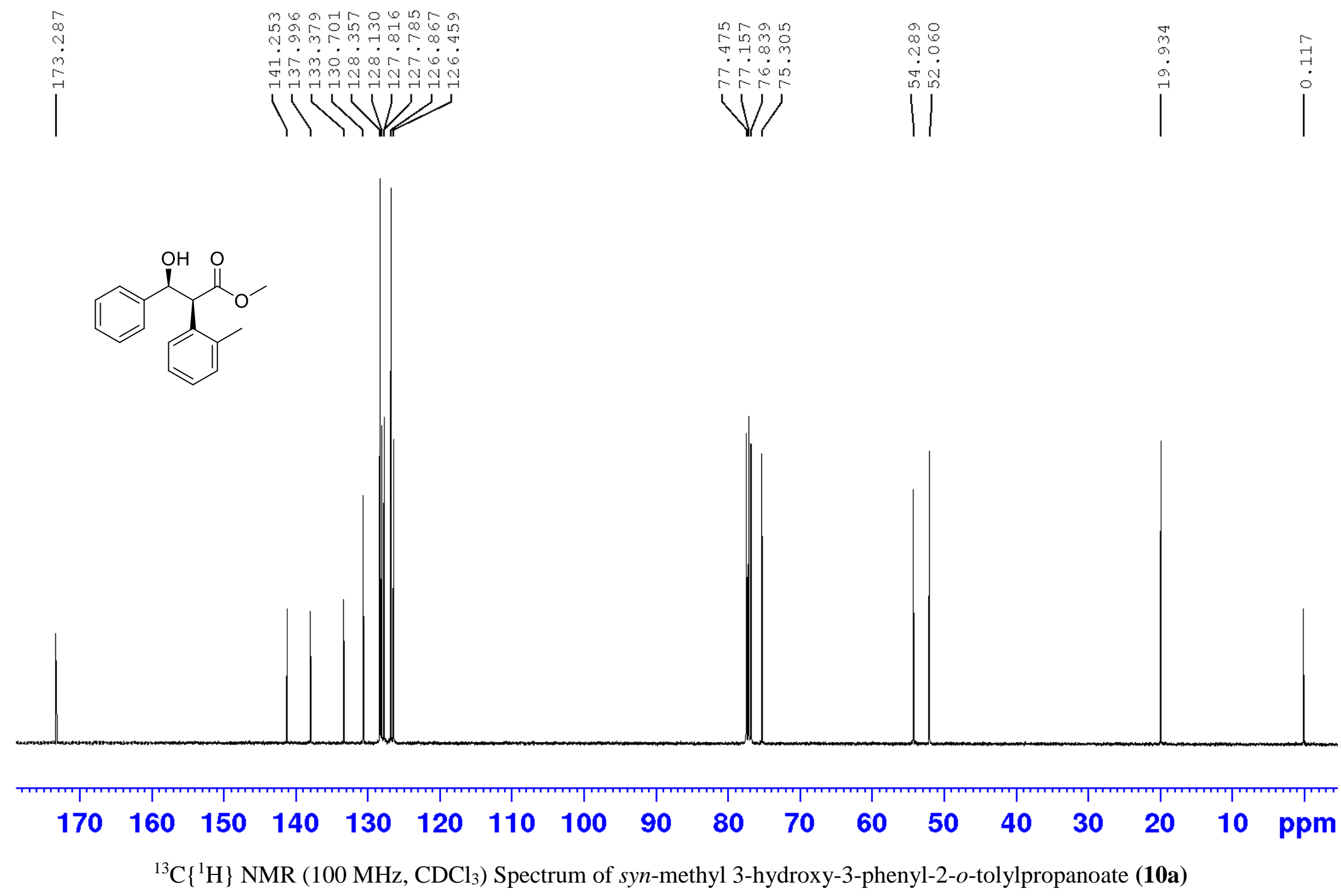
개의

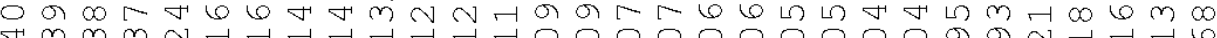
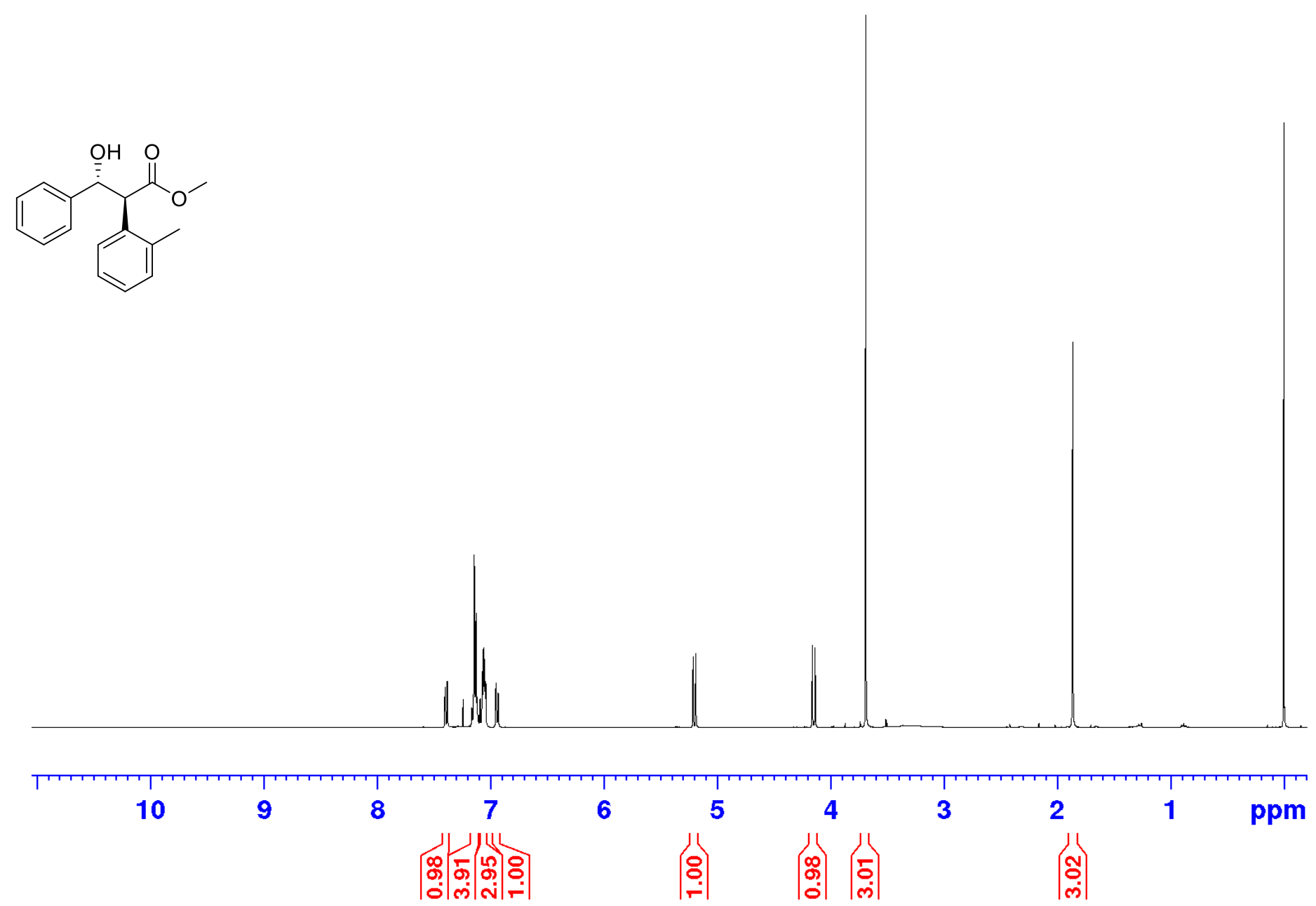

${ }^{1} \mathrm{H}$ NMR (400 MHz, $\mathrm{CDCl}_{3}$ ) Spectrum of anti-methyl 3-hydroxy-3-phenyl-2-o-tolylpropanoate (10a) 


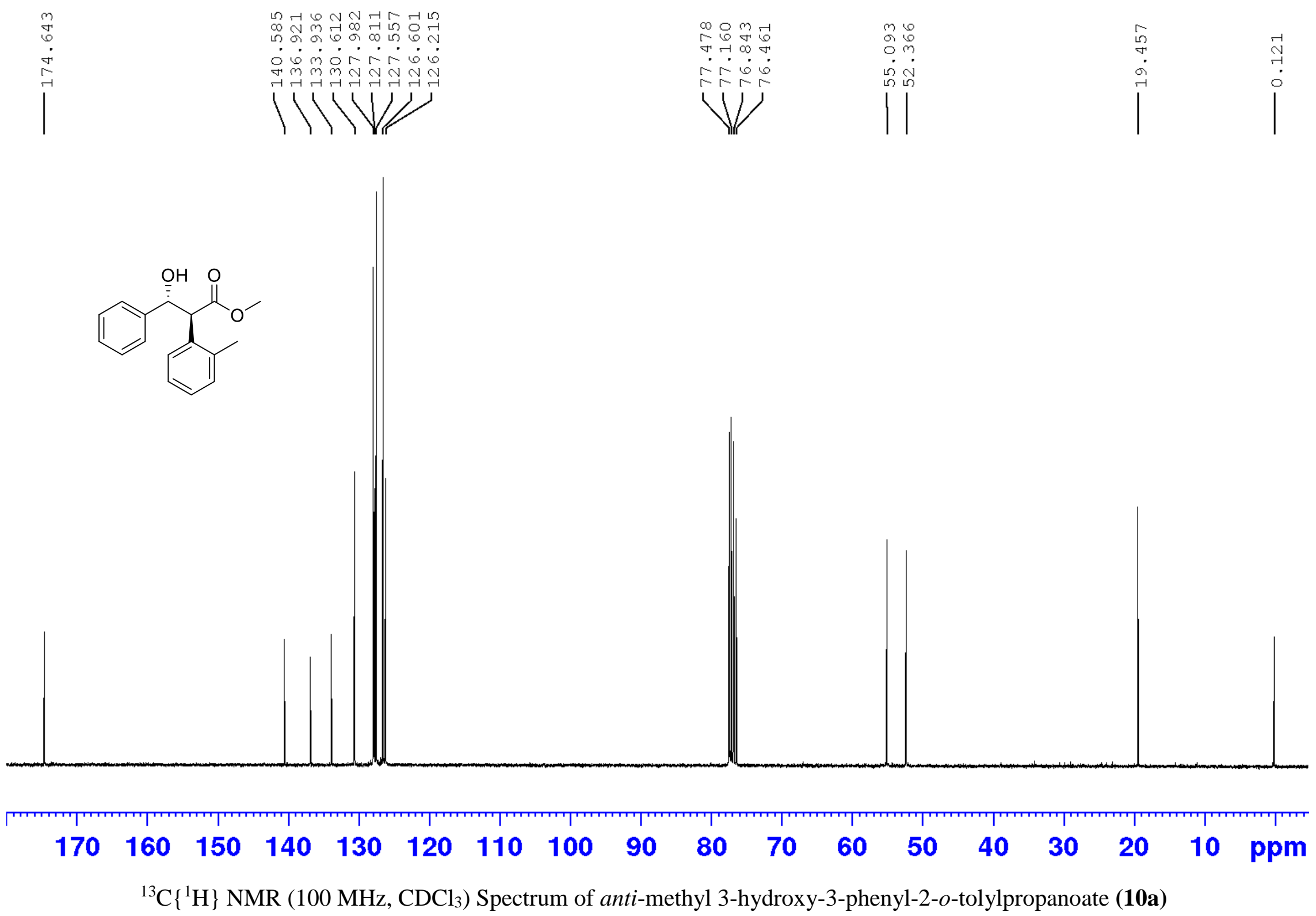


అ

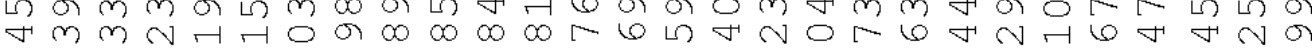
m m m $m$ m

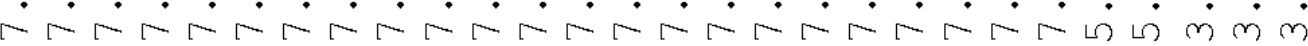

\section{$L=b=0$}

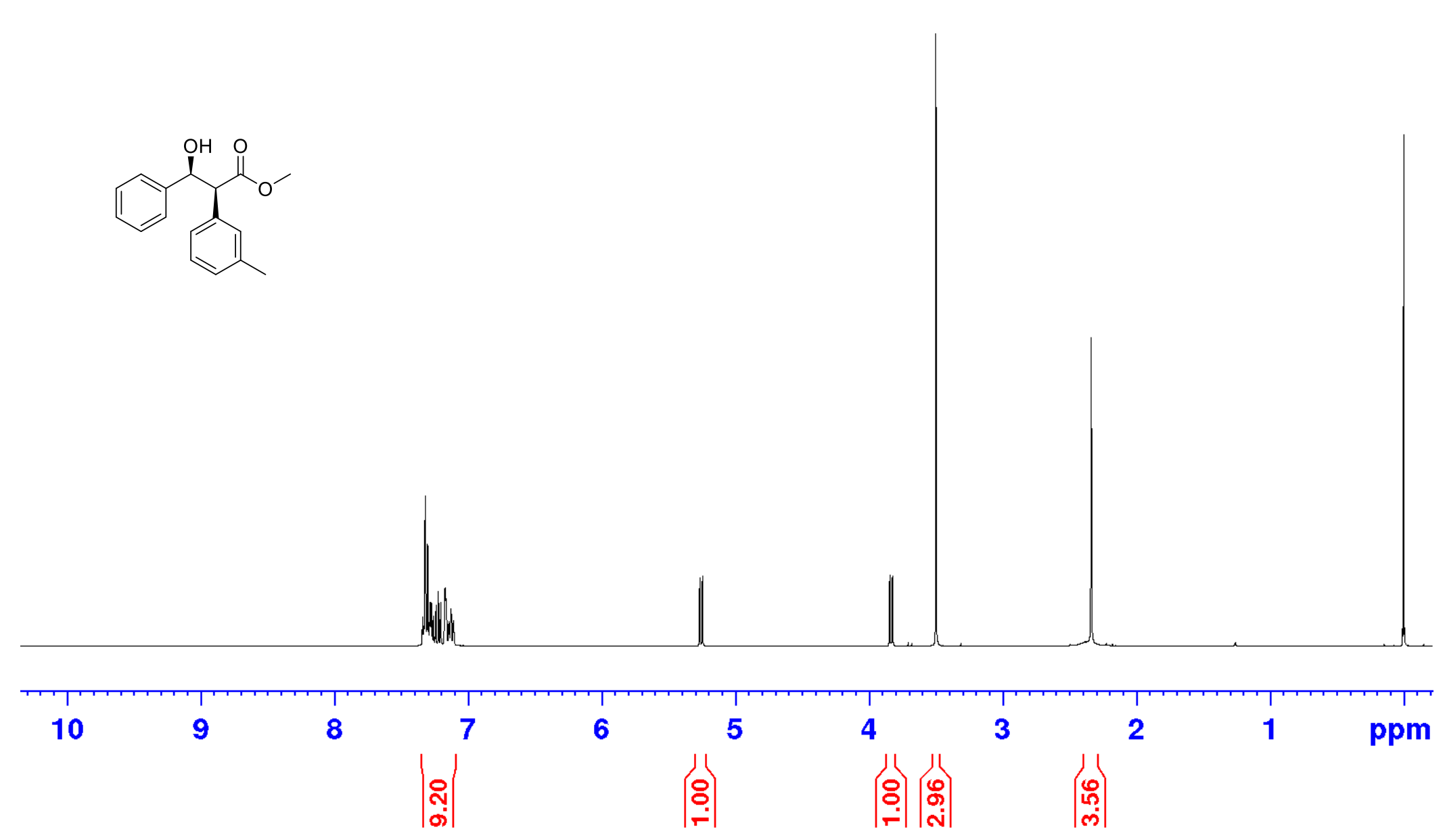

${ }^{1} \mathrm{H}$ NMR (400 MHz, $\mathrm{CDCl}_{3}$ ) Spectrum of syn-methyl 3-hydroxy-3-phenyl-2-m-tolylpropanoate (10b) 


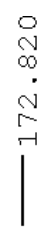
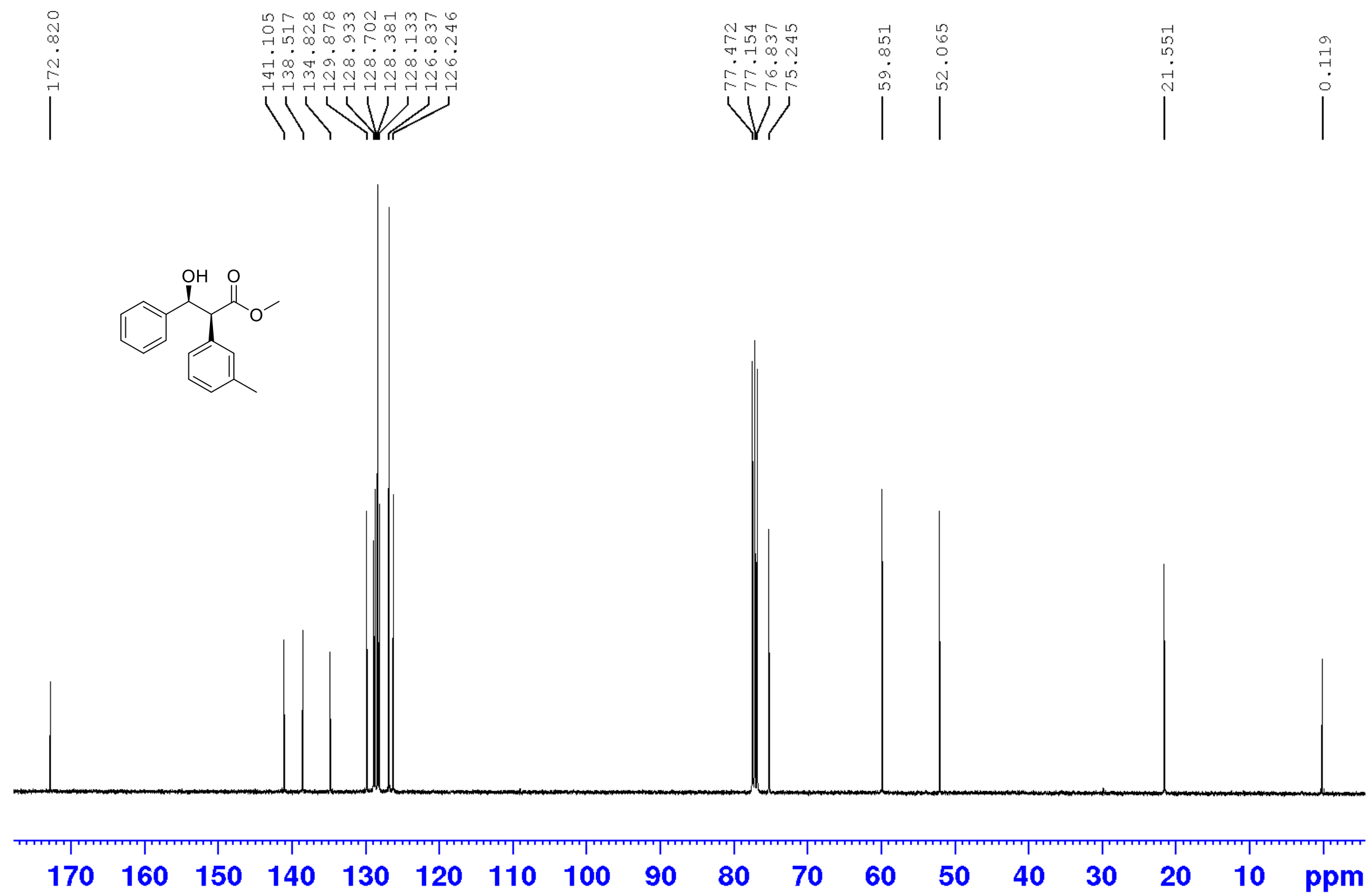

${ }^{13} \mathrm{C}\left\{{ }^{1} \mathrm{H}\right\}$ NMR $\left(100 \mathrm{MHz}, \mathrm{CDCl}_{3}\right)$ Spectrum of syn-methyl 3-hydroxy-3-phenyl-2-m-tolylpropanoate (10b)

S47 


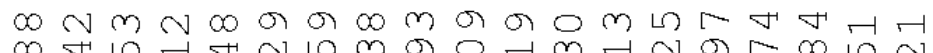
$\infty$ 小

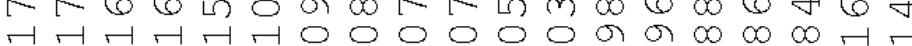

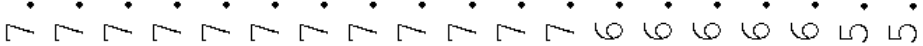
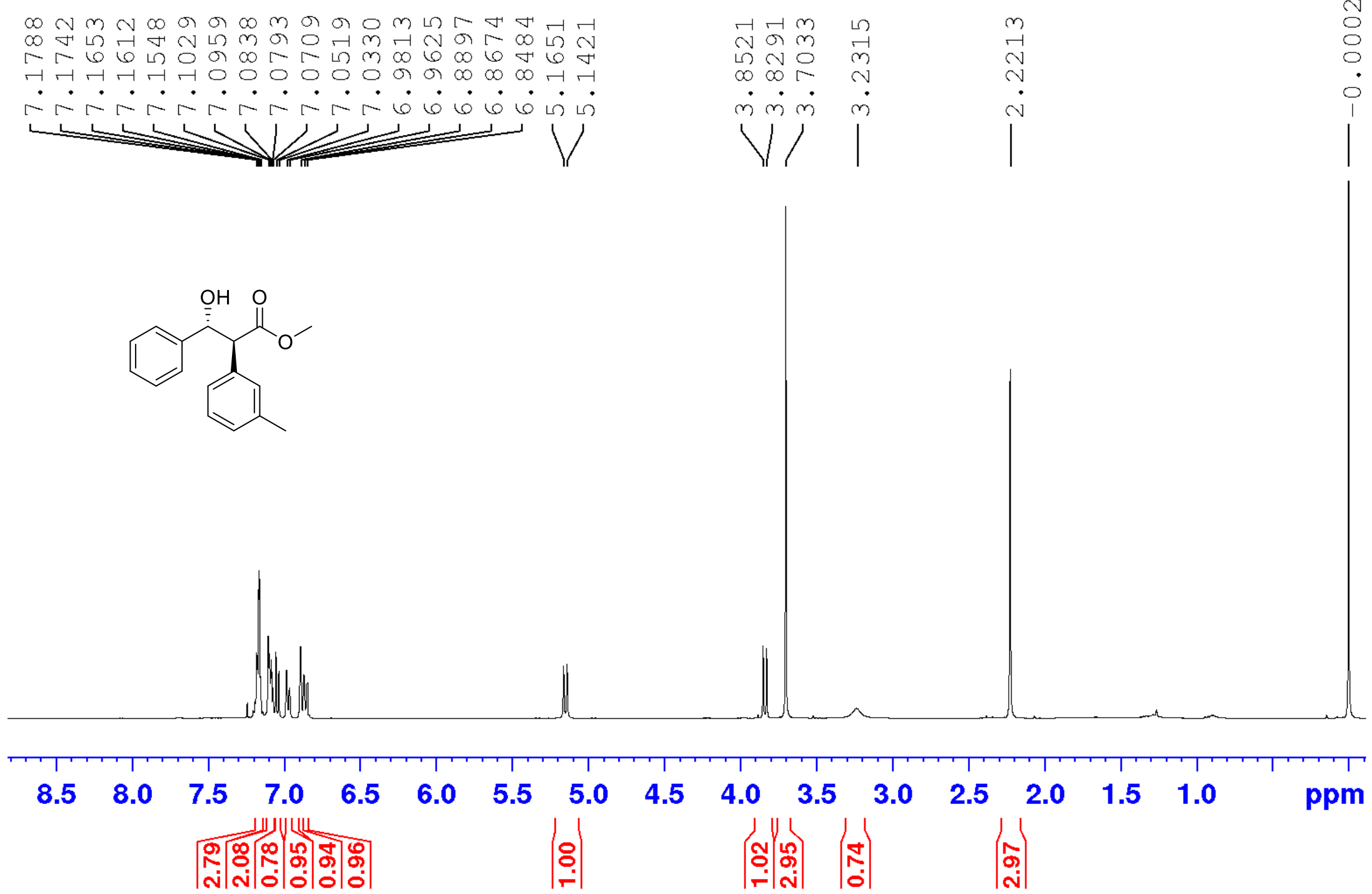

${ }^{1} \mathrm{H}$ NMR (400 MHz, $\mathrm{CDCl}_{3}$ ) Spectrum of anti-methyl 3-hydroxy-3-phenyl-2-m-tolylpropanoate (10b) 

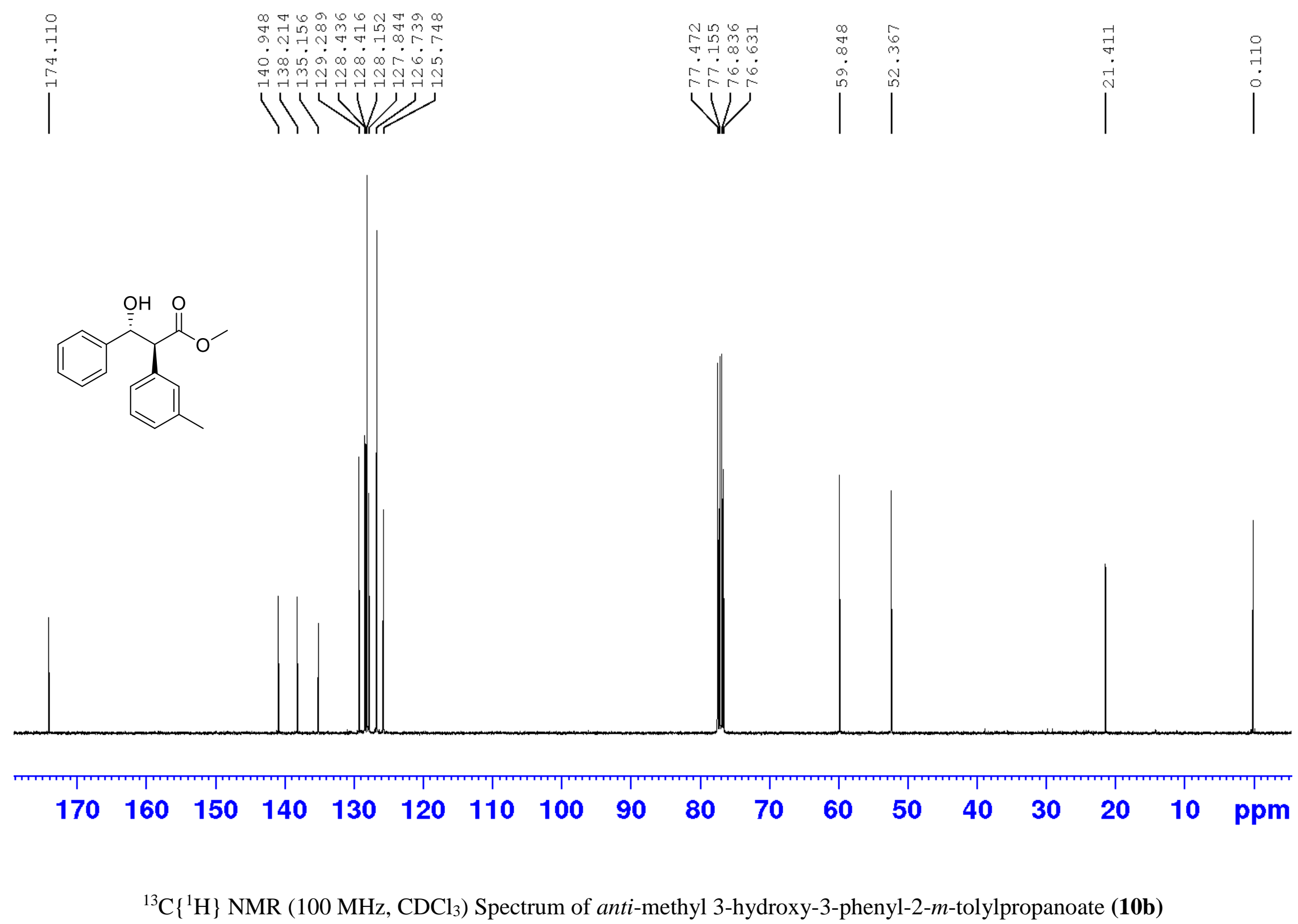

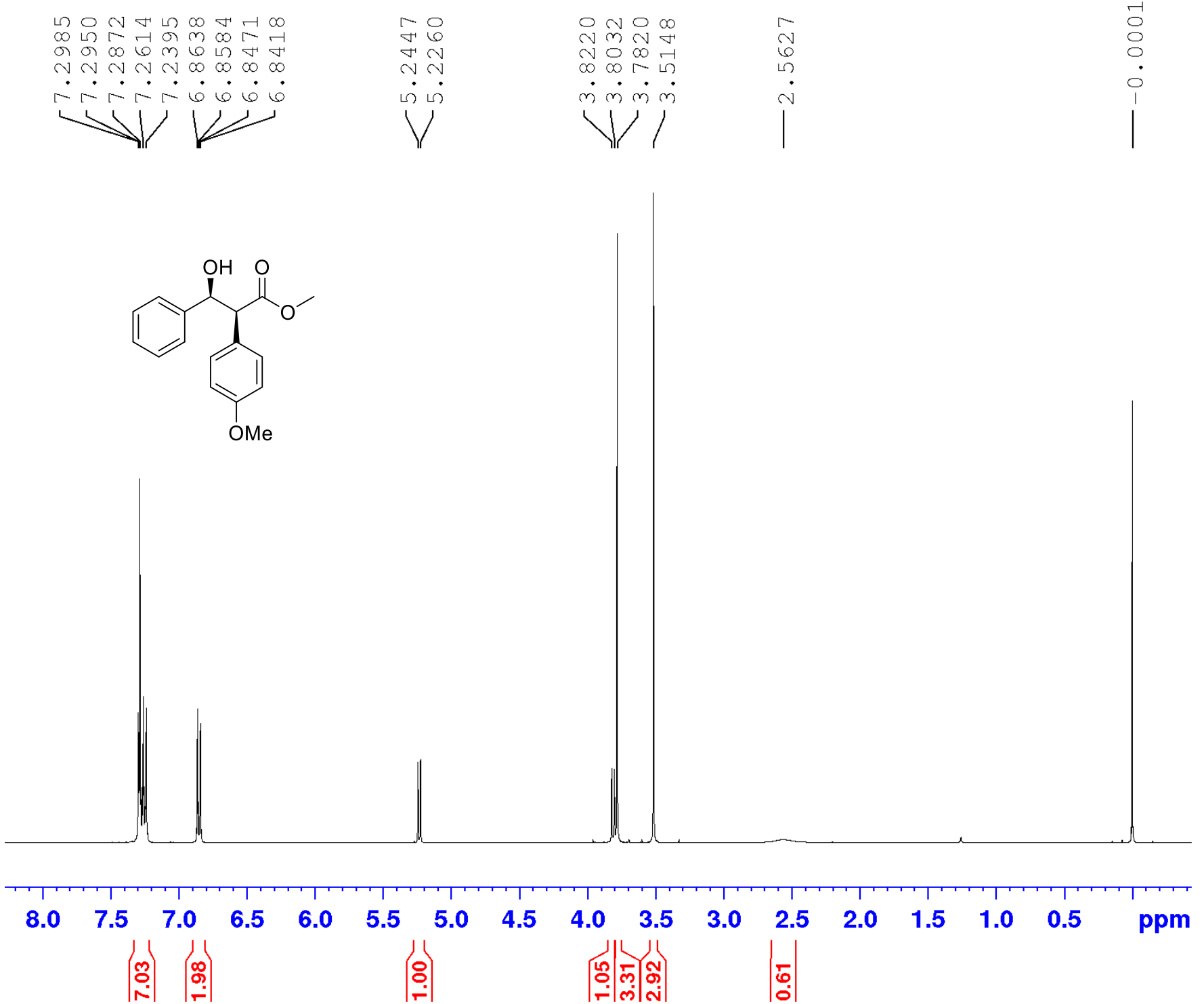

${ }^{1} \mathrm{H}$ NMR (400 MHz, $\mathrm{CDCl}_{3}$ ) Spectrum of syn-methyl 3-hydroxy-2-(4-methoxyphenyl)-3-phenylpropanoate (10c) 

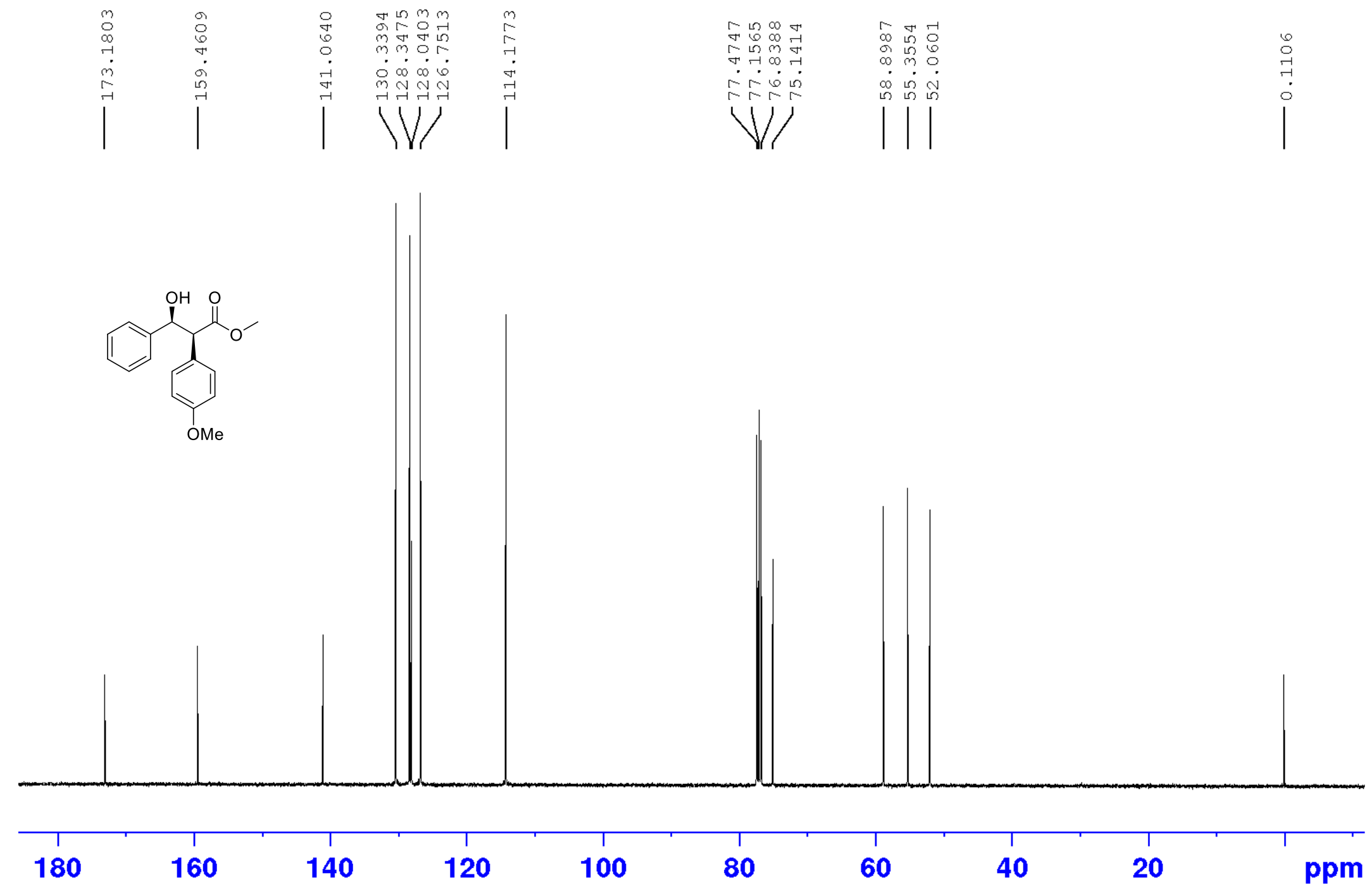

${ }^{13} \mathrm{C}\left\{{ }^{1} \mathrm{H}\right\}$ NMR (100 MHz, $\left.\mathrm{CDCl}_{3}\right)$ Spectrum of syn-methyl 3-hydroxy-2-(4-methoxyphenyl)-3-phenylpropanoate (10c) 


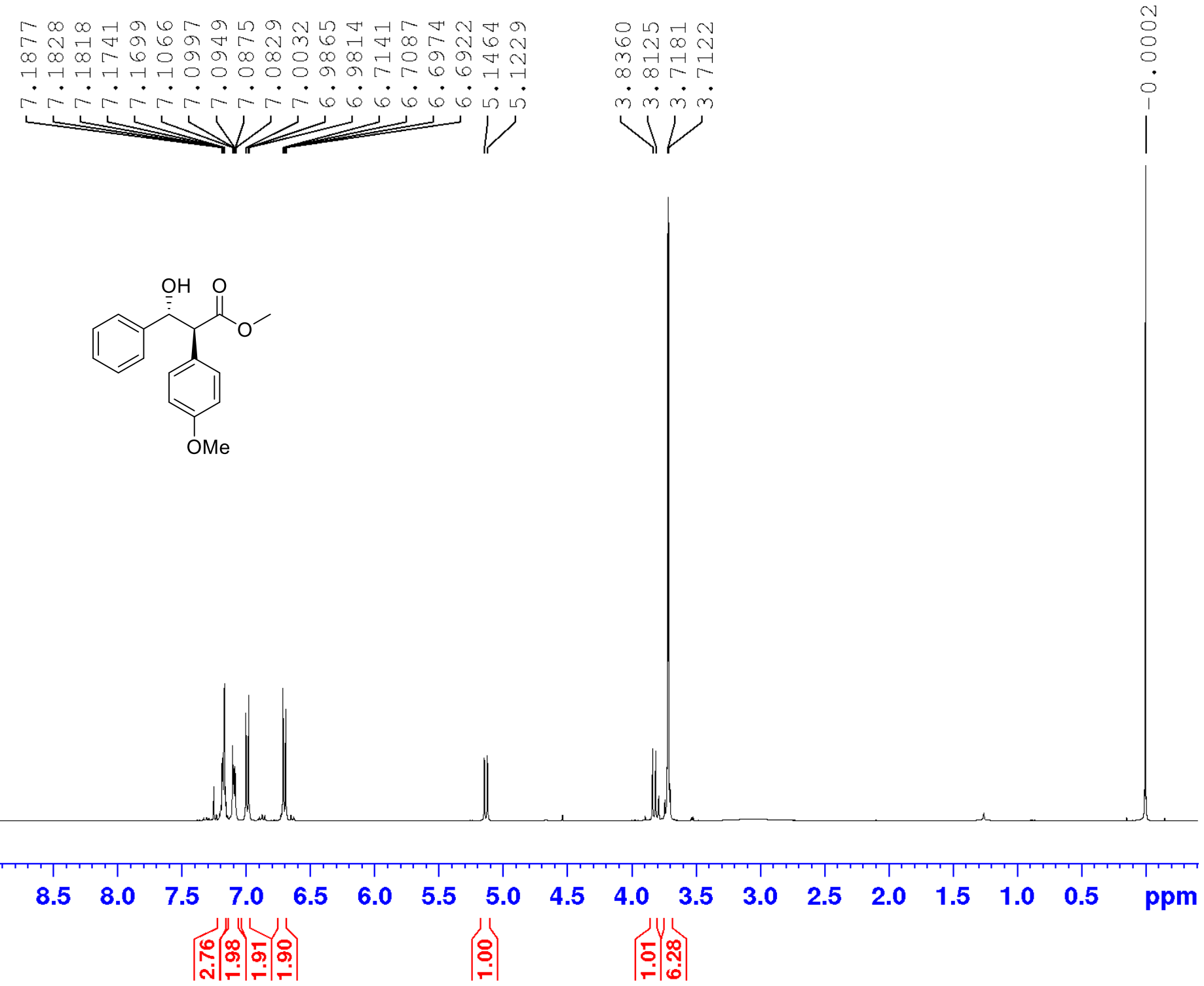

${ }^{1} \mathrm{H}$ NMR (400 MHz, $\mathrm{CDCl}_{3}$ ) Spectrum of anti-methyl 3-hydroxy-2-(4- methoxyphenyl)-3-phenylpropanoate (10c) 

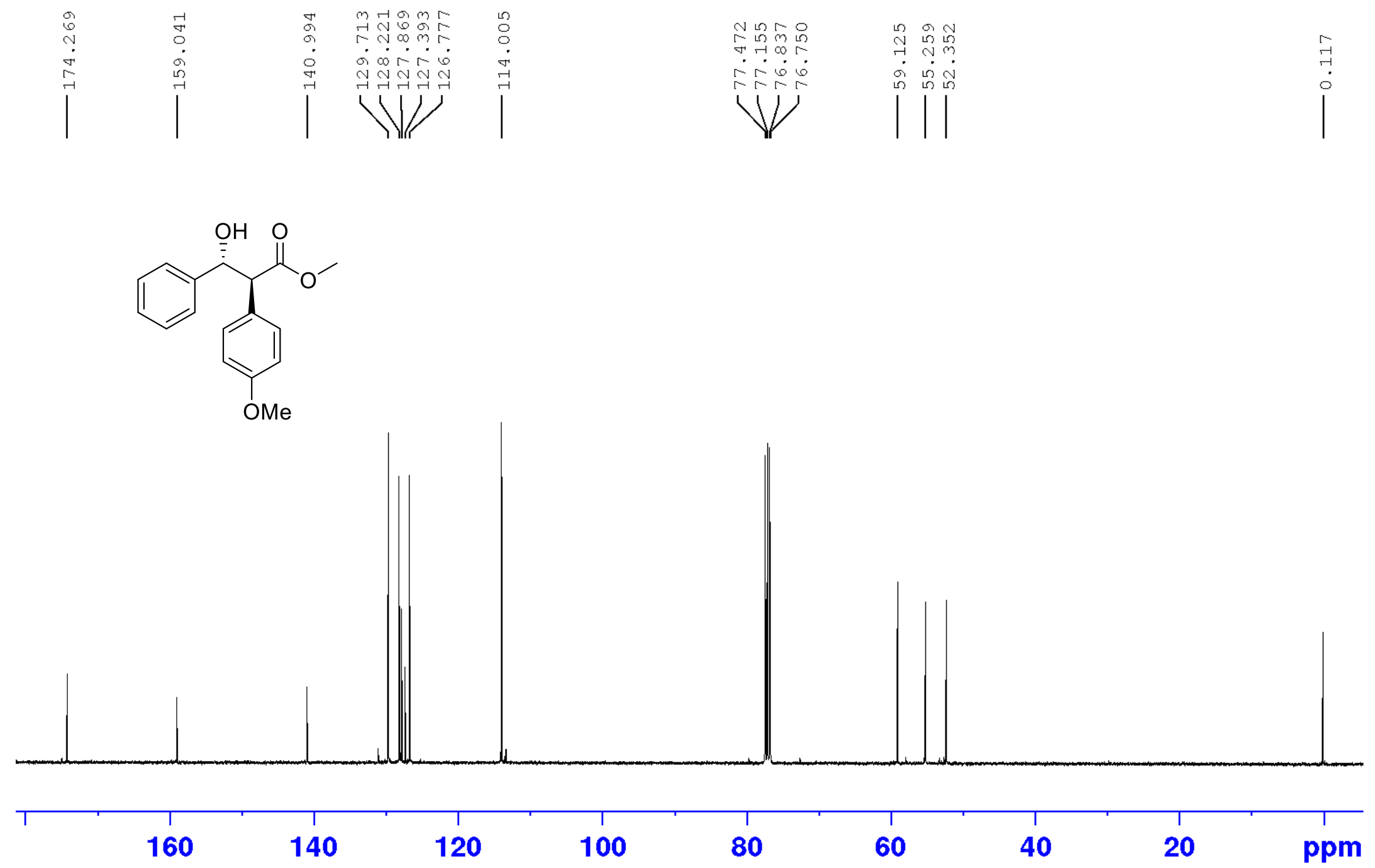

${ }^{13} \mathrm{C}\left\{{ }^{1} \mathrm{H}\right\}$ NMR $\left(100 \mathrm{MHz}, \mathrm{CDCl}_{3}\right)$ Spectrum of anti-methyl 3-hydroxy-2-(4-methoxyphenyl)-3-phenylpropanoate (10c) 
小 小० m M N N N N N N

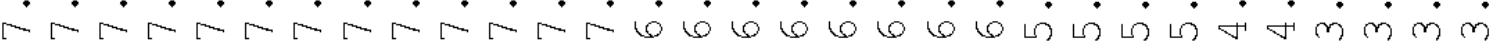
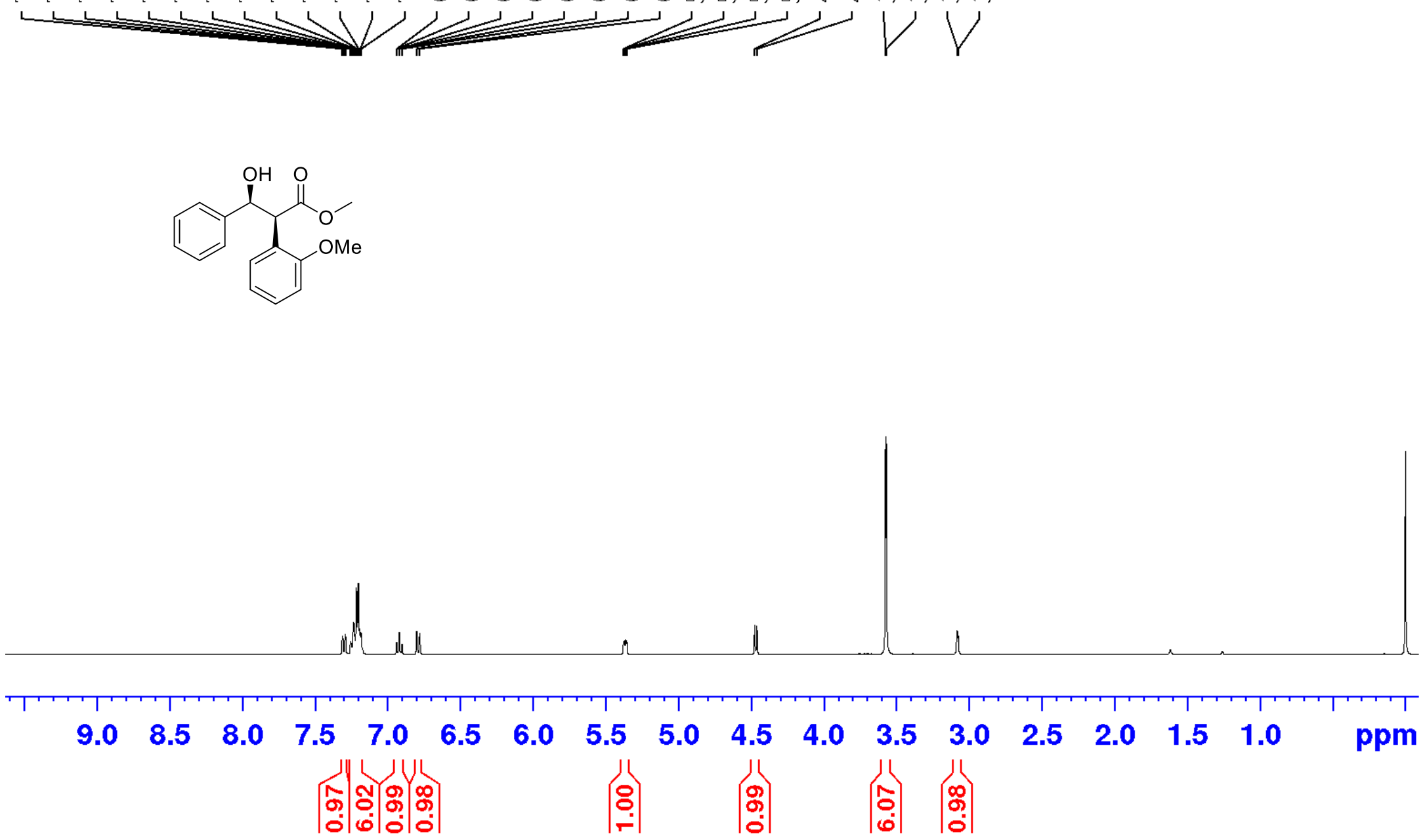

${ }^{1} \mathrm{H}$ NMR (400 MHz, $\mathrm{CDCl}_{3}$ ) Spectrum of syn-methyl 3-hydroxy-2-(2-methoxyphenyl)-3-phenylpropanoate (10d) 

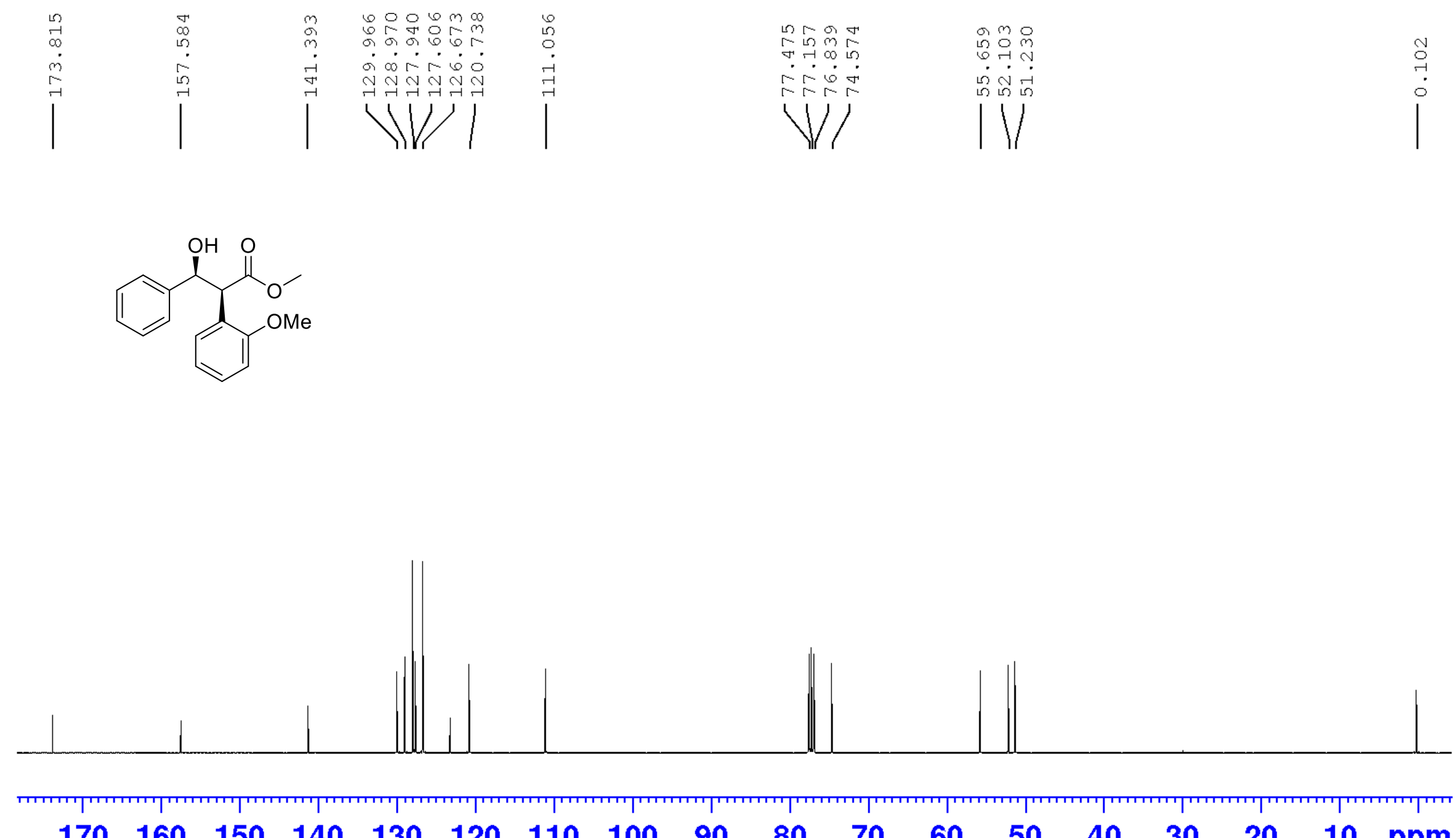

$\begin{array}{llllllllllllllllll}170 & 160 & 150 & 140 & 130 & 120 & 110 & 100 & 90 & 80 & 70 & 60 & 50 & 40 & 30 & 20 & 10 & \mathrm{ppm}\end{array}$

${ }^{13} \mathrm{C}\left\{{ }^{1} \mathrm{H}\right\}$ NMR (100 MHz, CDCl 3 ) Spectrum of syn-methyl 3-hydroxy-2-(2-methoxyphenyl)-3-phenylpropanoate (10d) 
๘

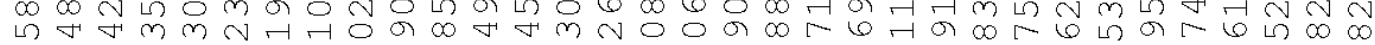
L

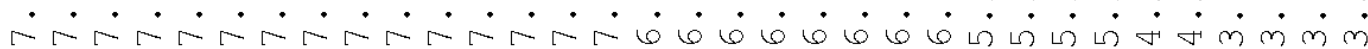
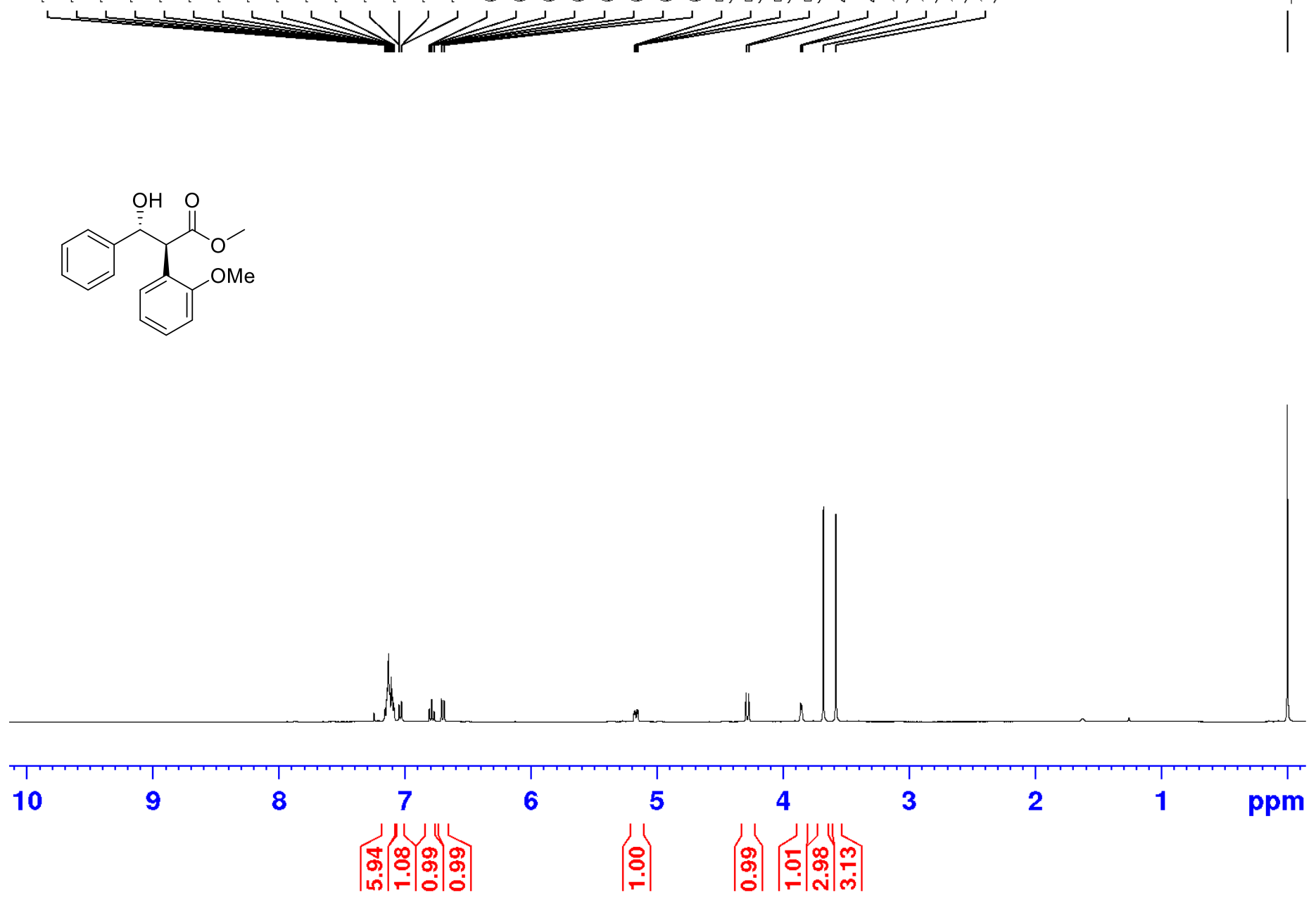

${ }^{1} \mathrm{H}$ NMR (400 MHz, $\mathrm{CDCl}_{3}$ ) Spectrum of anti-methyl 3-hydroxy-2-(2-methoxyphenyl)-3-phenylpropanoate (10d) 


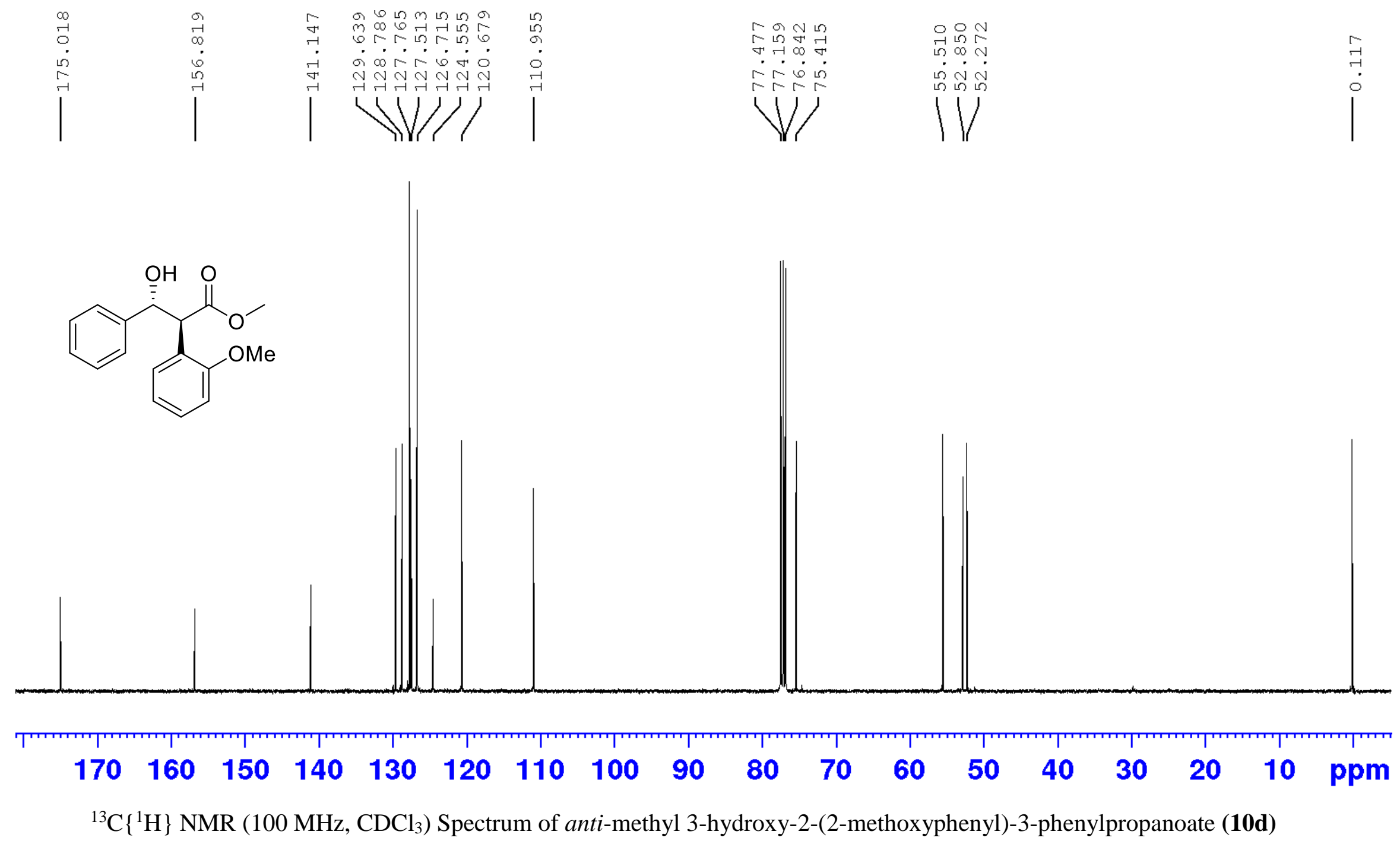


m

अ

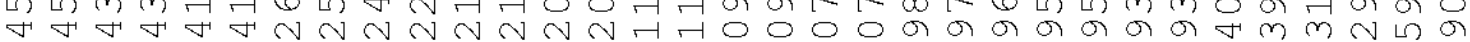

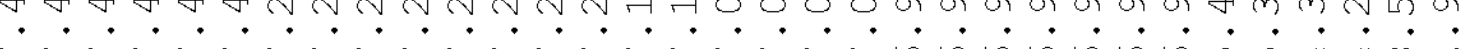

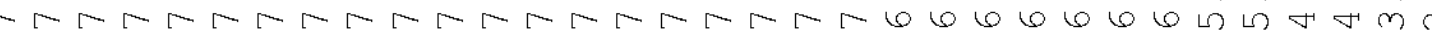

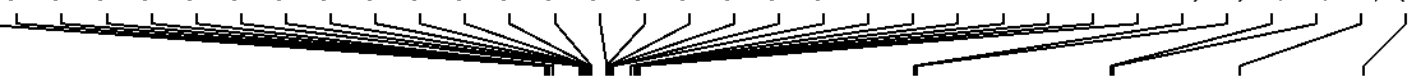
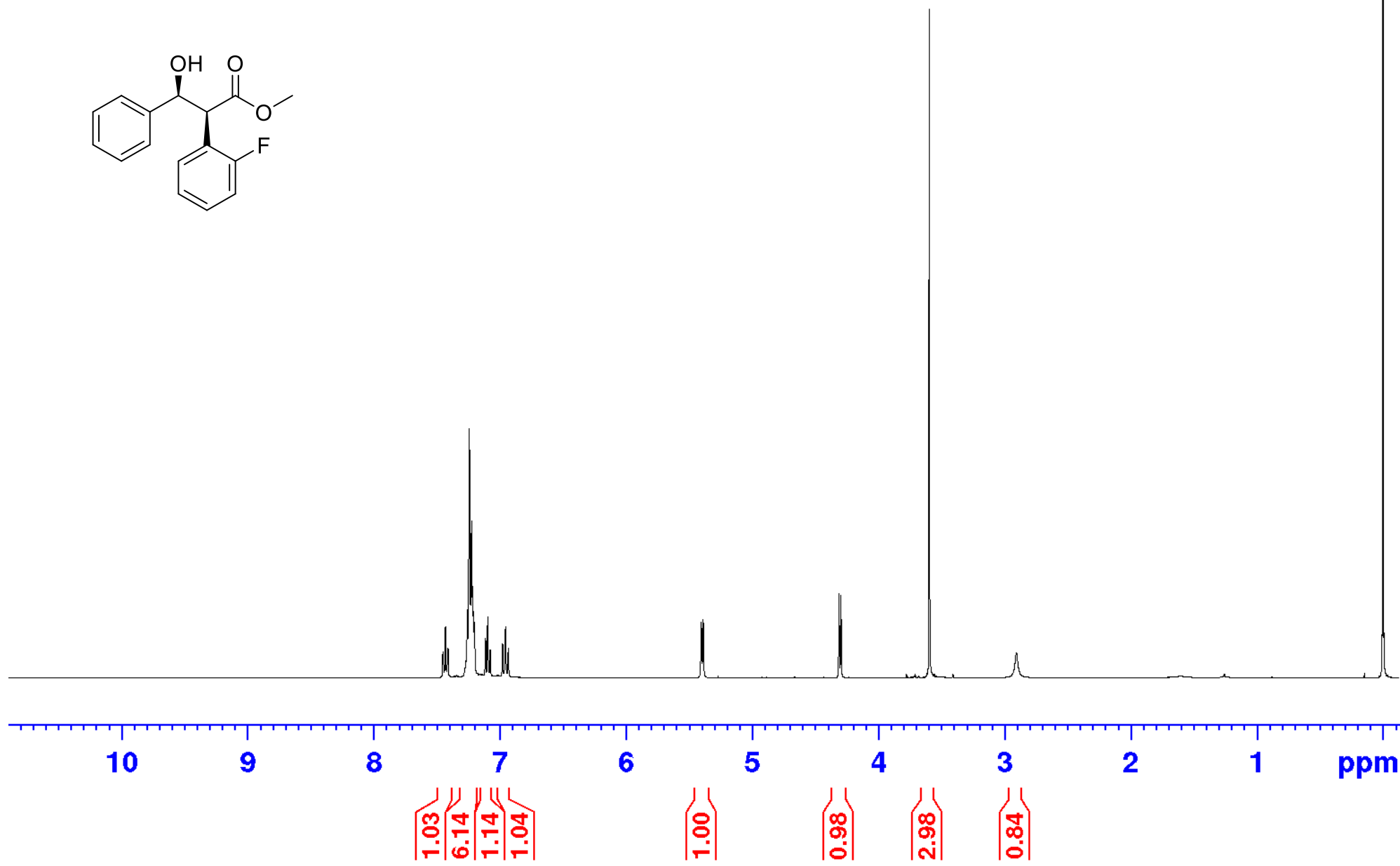

${ }^{1} \mathrm{H} \mathrm{NMR}\left(400 \mathrm{MHz}, \mathrm{CDCl}_{3}\right.$ ) Spectrum of syn-methyl 2-(2-fluorophenyl)-3-hydroxy-3-phenylpropanoate (10e) 


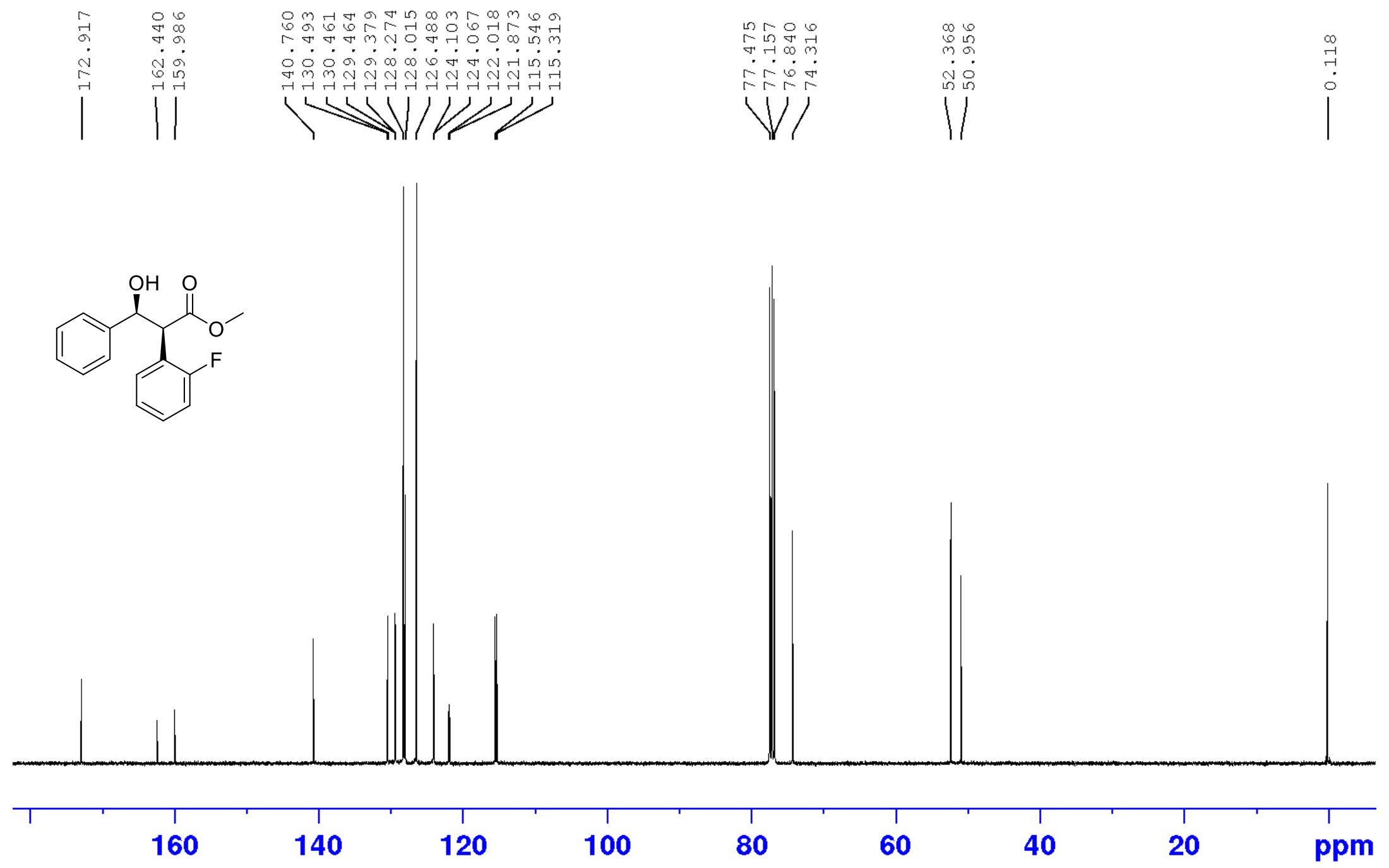

${ }^{13} \mathrm{C}\left\{{ }^{1} \mathrm{H}\right\}$ NMR (100 MHz, $\mathrm{CDCl}_{3}$ ) Spectrum of syn-methyl 2-(2-fluorophenyl)-3-hydroxy-3-phenylpropanoate (10e) 
m 가잉 N N

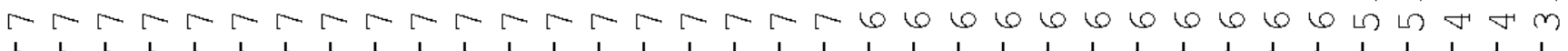

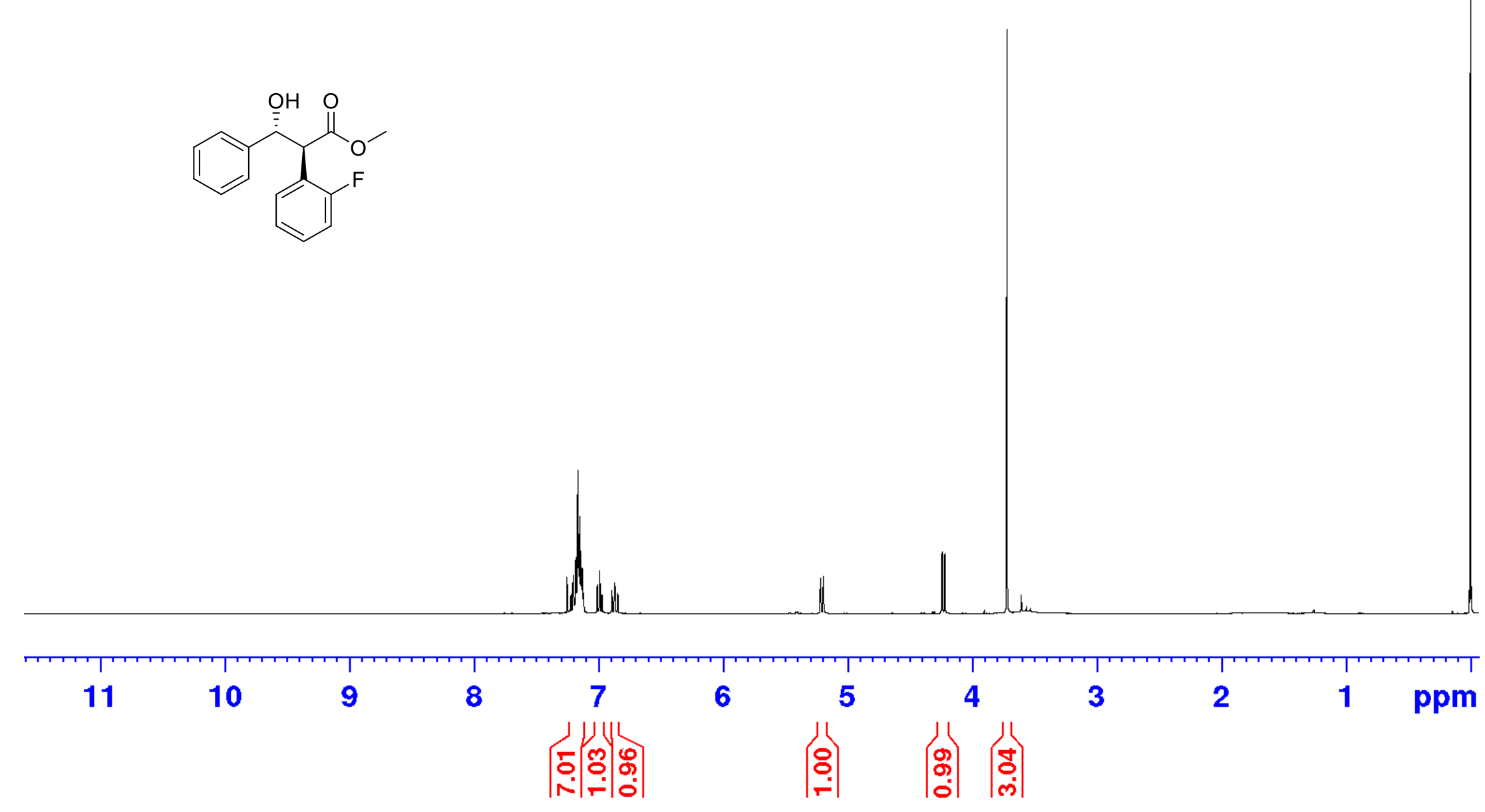

${ }^{1} \mathrm{H}$ NMR (400 MHz, $\mathrm{CDCl}_{3}$ ) Spectrum of anti-methyl 2-(2-fluorophenyl)-3-hydroxy-3-phenylpropanoate (10e) 

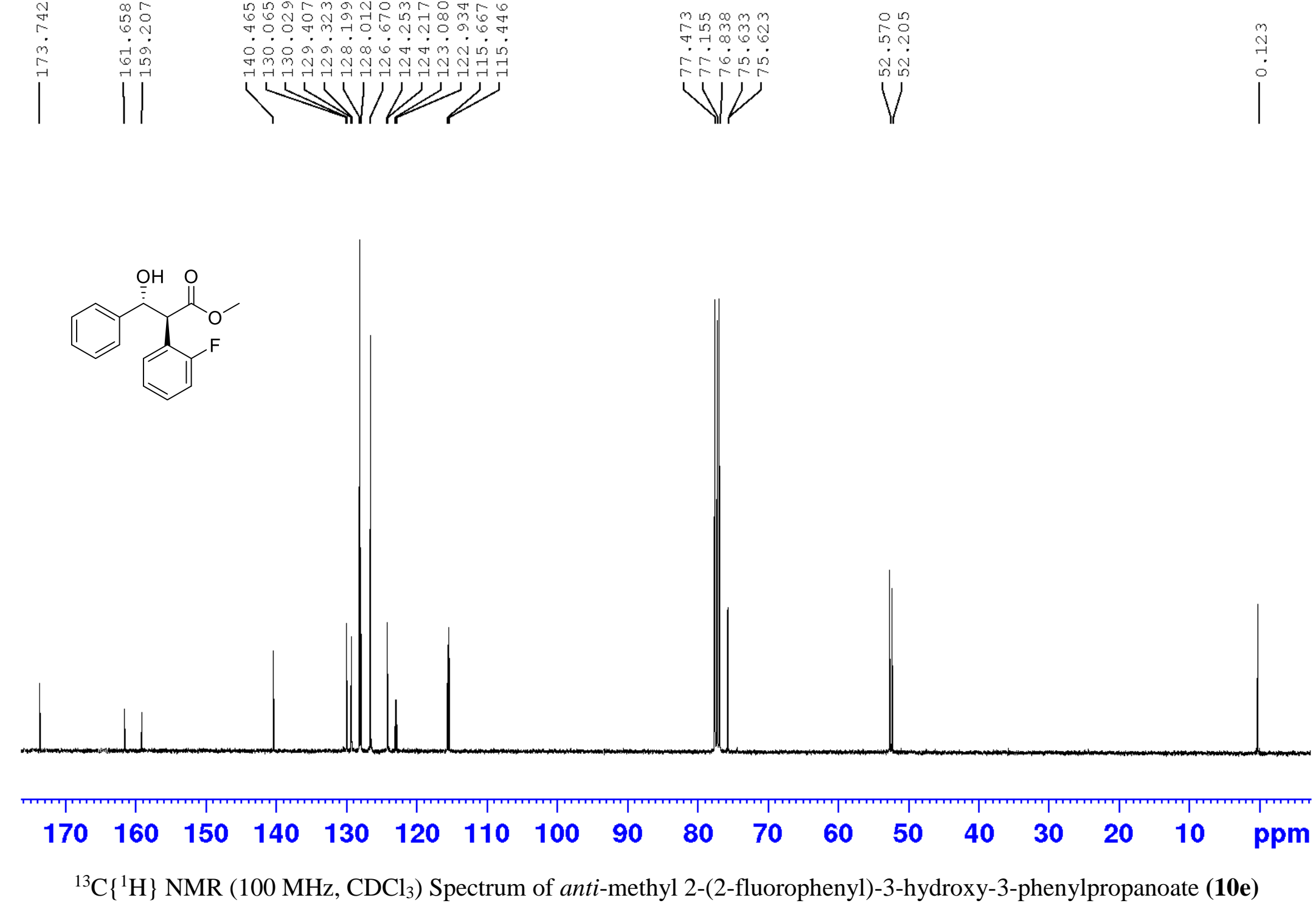

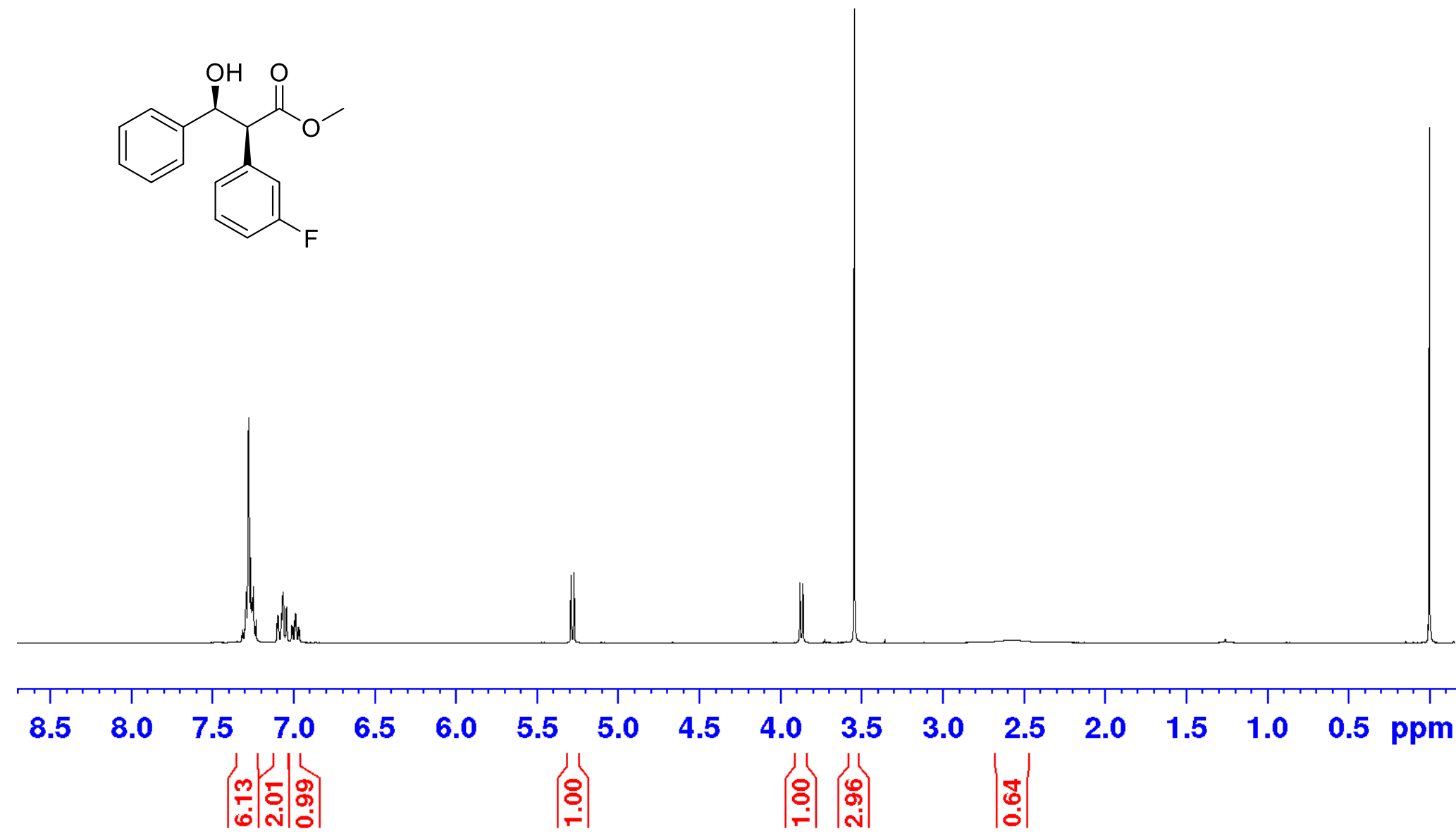

${ }^{1} \mathrm{H} \mathrm{NMR}\left(400 \mathrm{MHz}, \mathrm{CDCl}_{3}\right.$ ) Spectrum of syn-methyl 2-(3-fluorophenyl)-3-hydroxy-3-phenylpropanoate (10f) 

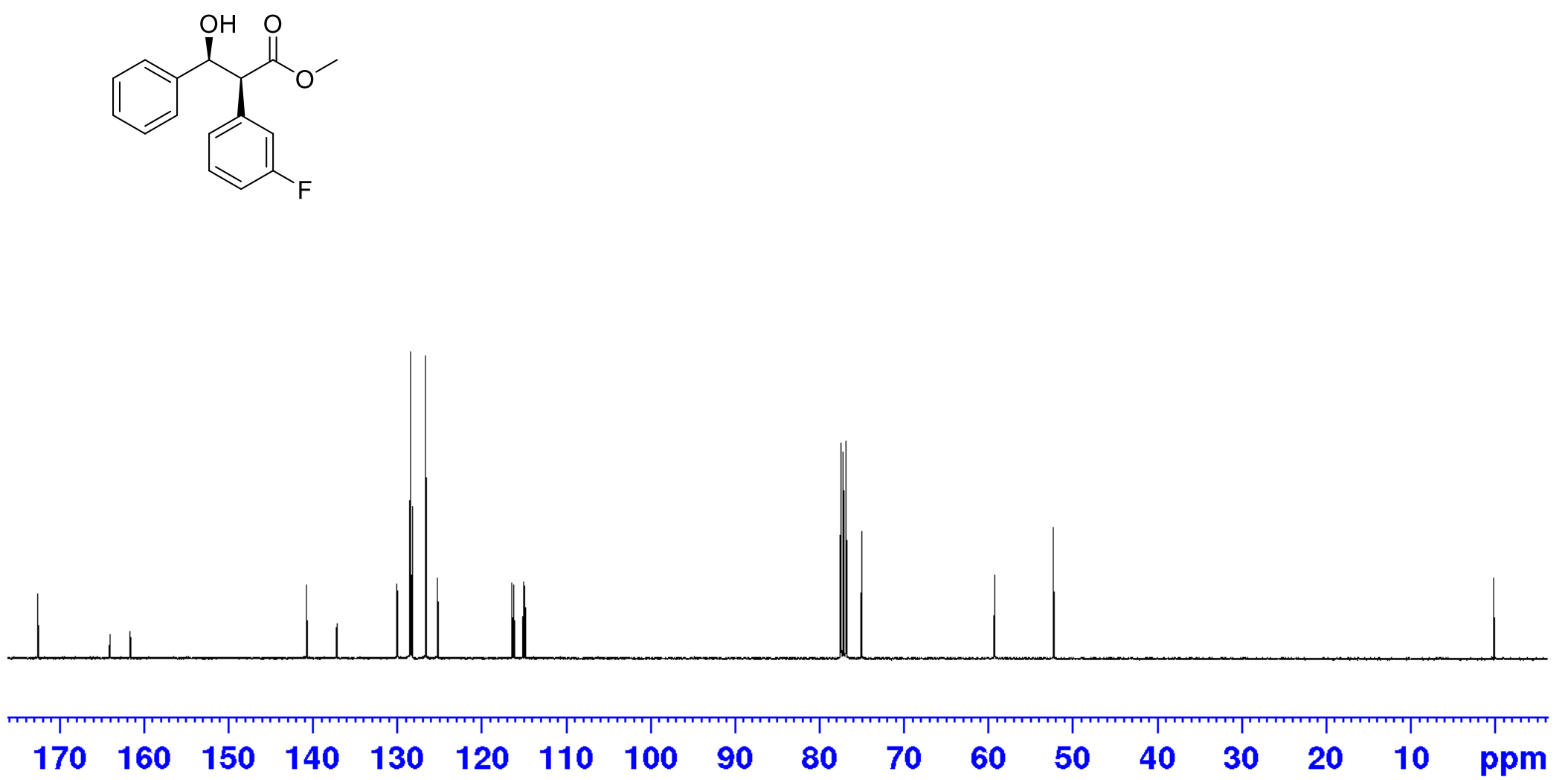

${ }^{13} \mathrm{C}\left\{{ }^{1} \mathrm{H}\right\}$ NMR (100 MHz, $\mathrm{CDCl}_{3}$ ) Spectrum of syn-methyl 2-(3-fluorophenyl)-3-hydroxy-3-phenylpropanoate (10f) 

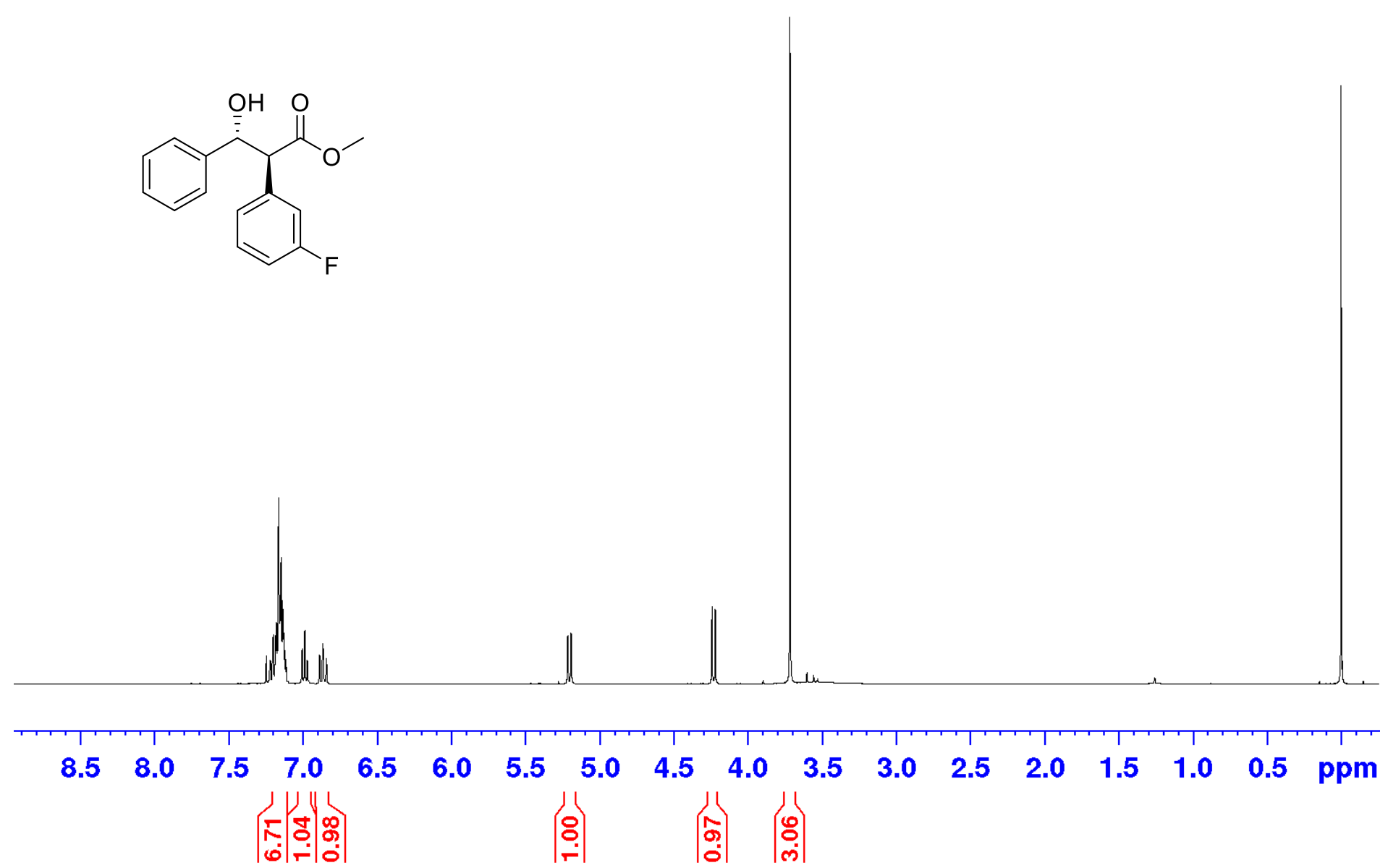

${ }^{1} \mathrm{H}$ NMR (400 MHz, $\mathrm{CDCl}_{3}$ ) Spectrum of anti-methyl 2-(3-fluorophenyl)-3-hydroxy-3-phenylpropanoate (10f) 

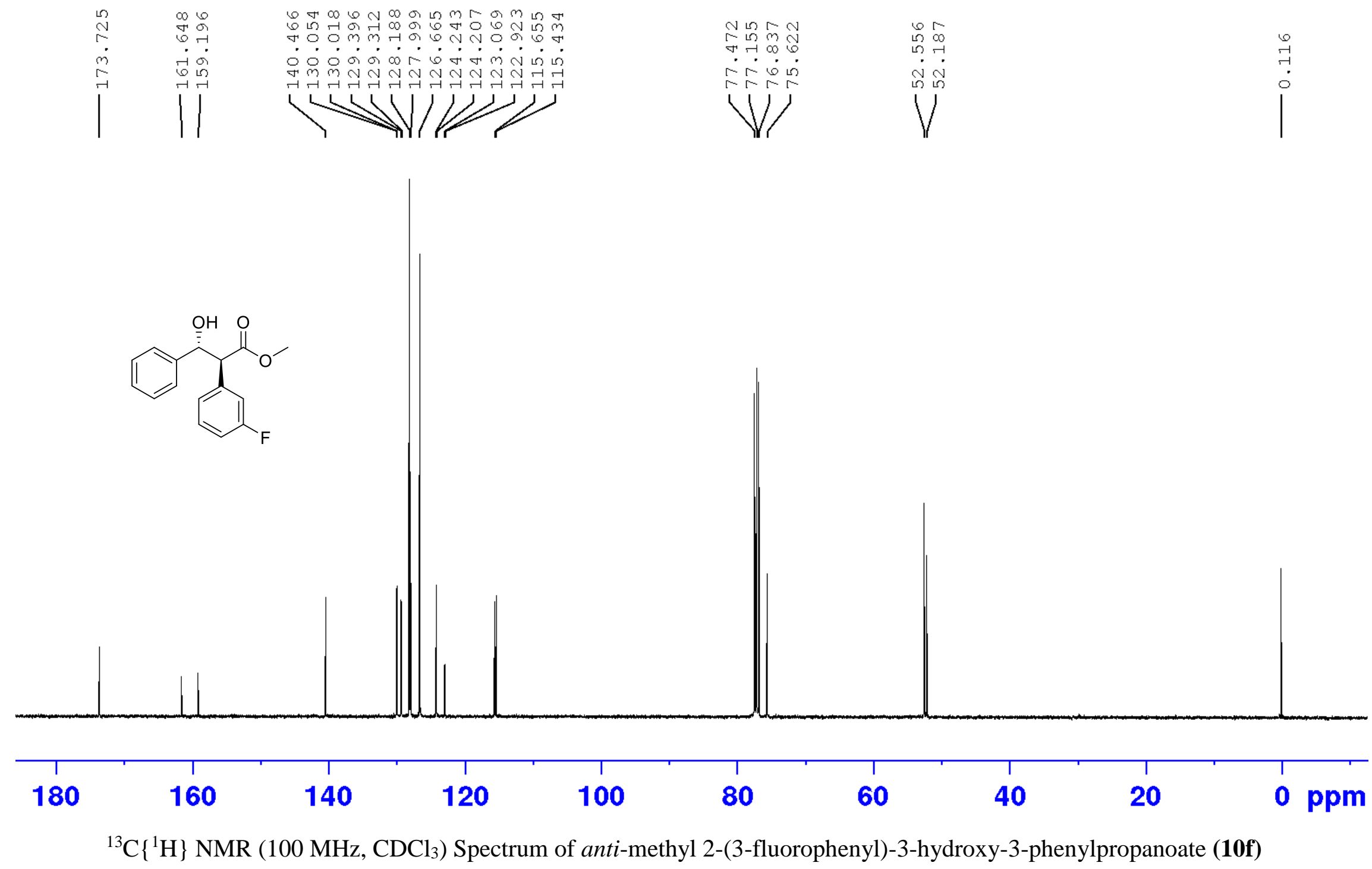


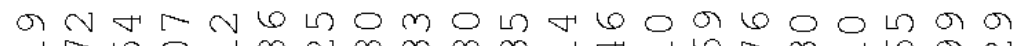
단

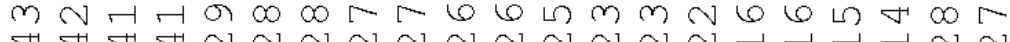
小

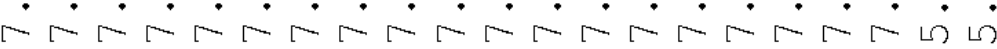
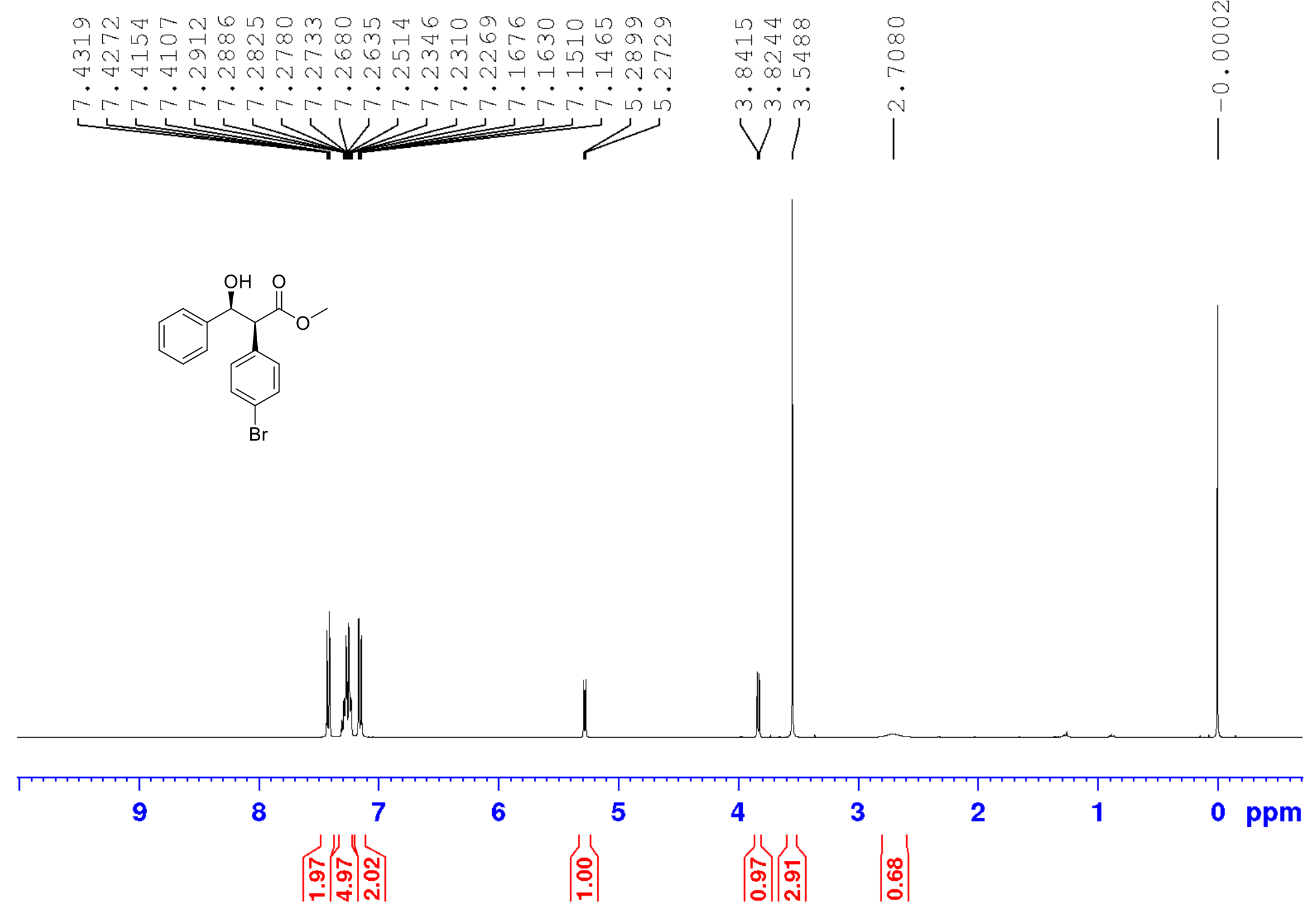

${ }^{1} \mathrm{H} \mathrm{NMR}\left(400 \mathrm{MHz}, \mathrm{CDCl}_{3}\right.$ ) Spectrum of syn-methyl 2-(4-bromophenyl)-3-hydroxy-3-phenylpropanoate (10g) 


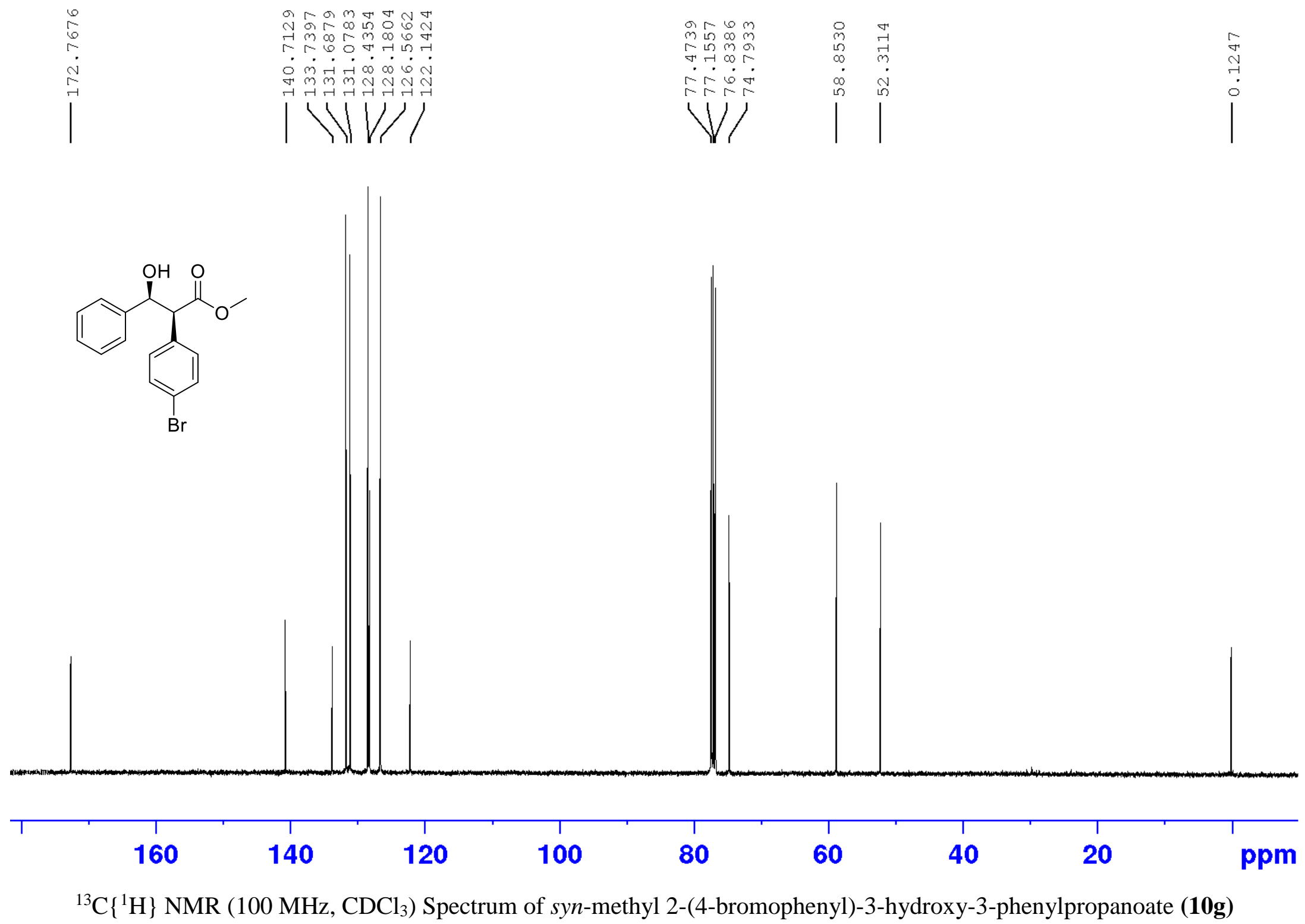


$\circ$ 구

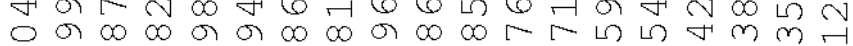

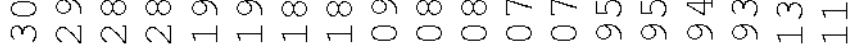

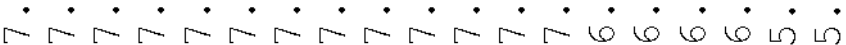
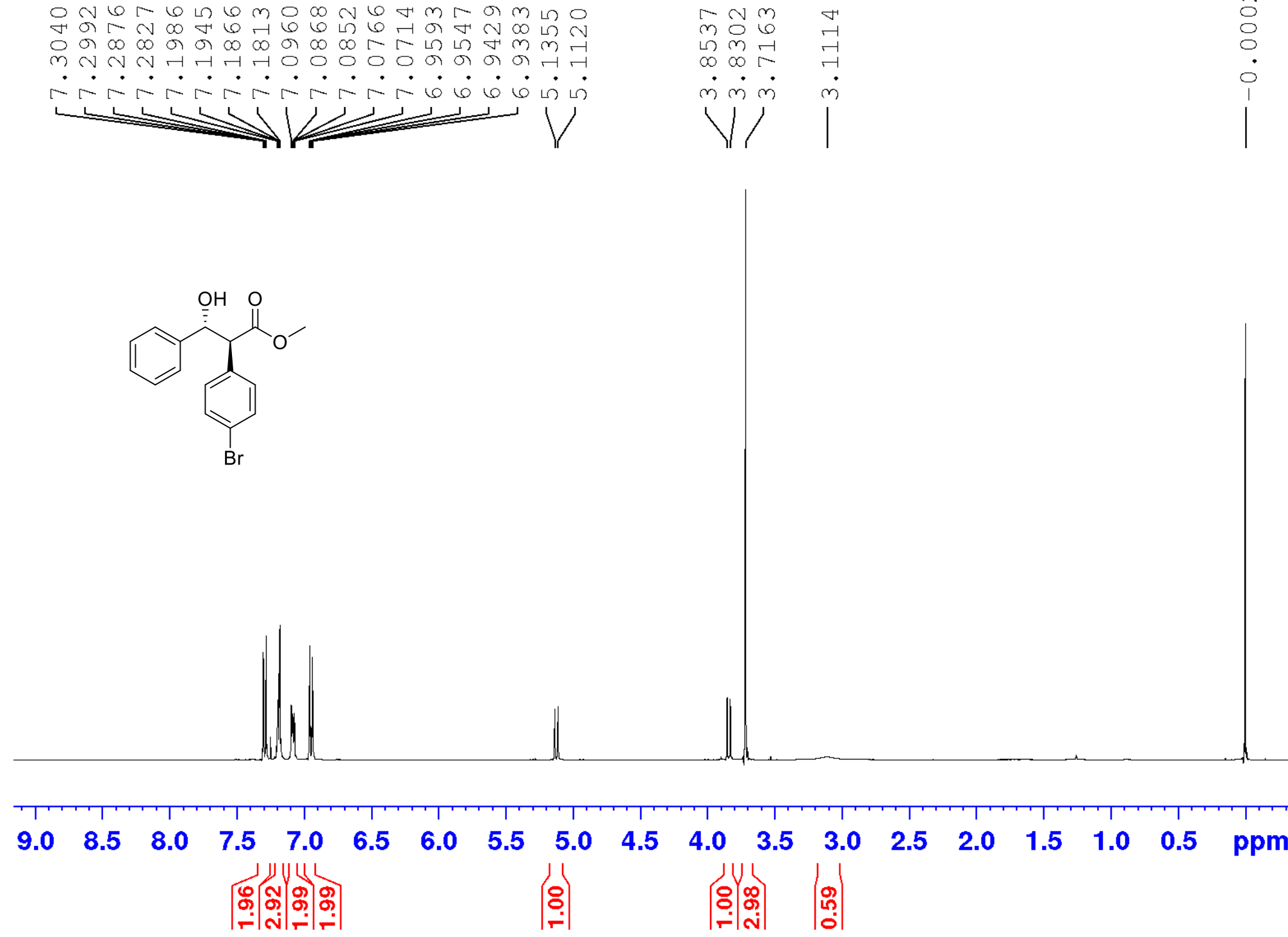

${ }^{1} \mathrm{H}$ NMR (400 MHz, $\mathrm{CDCl}_{3}$ ) Spectrum of anti-methyl 2-(4-bromophenyl)-3-hydroxy-3-phenylpropanoate (10g) 


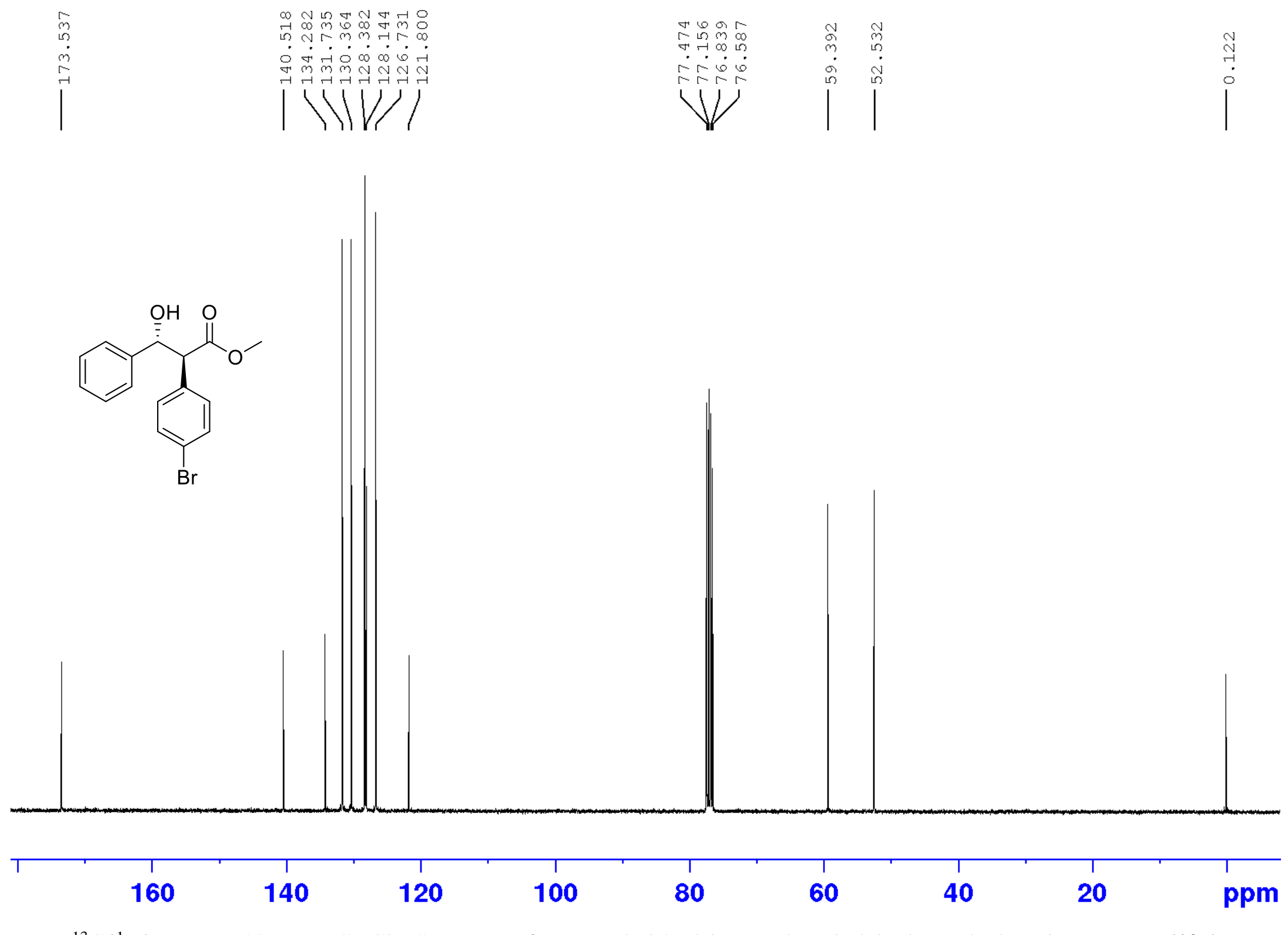

${ }^{13} \mathrm{C}\left\{{ }^{1} \mathrm{H}\right\} \mathrm{NMR}\left(100 \mathrm{MHz}, \mathrm{CDCl}_{3}\right)$ Spectrum of anti-methyl 2-(4-bromophenyl)-3-hydroxy-3-phenylpropanoate (10g) 

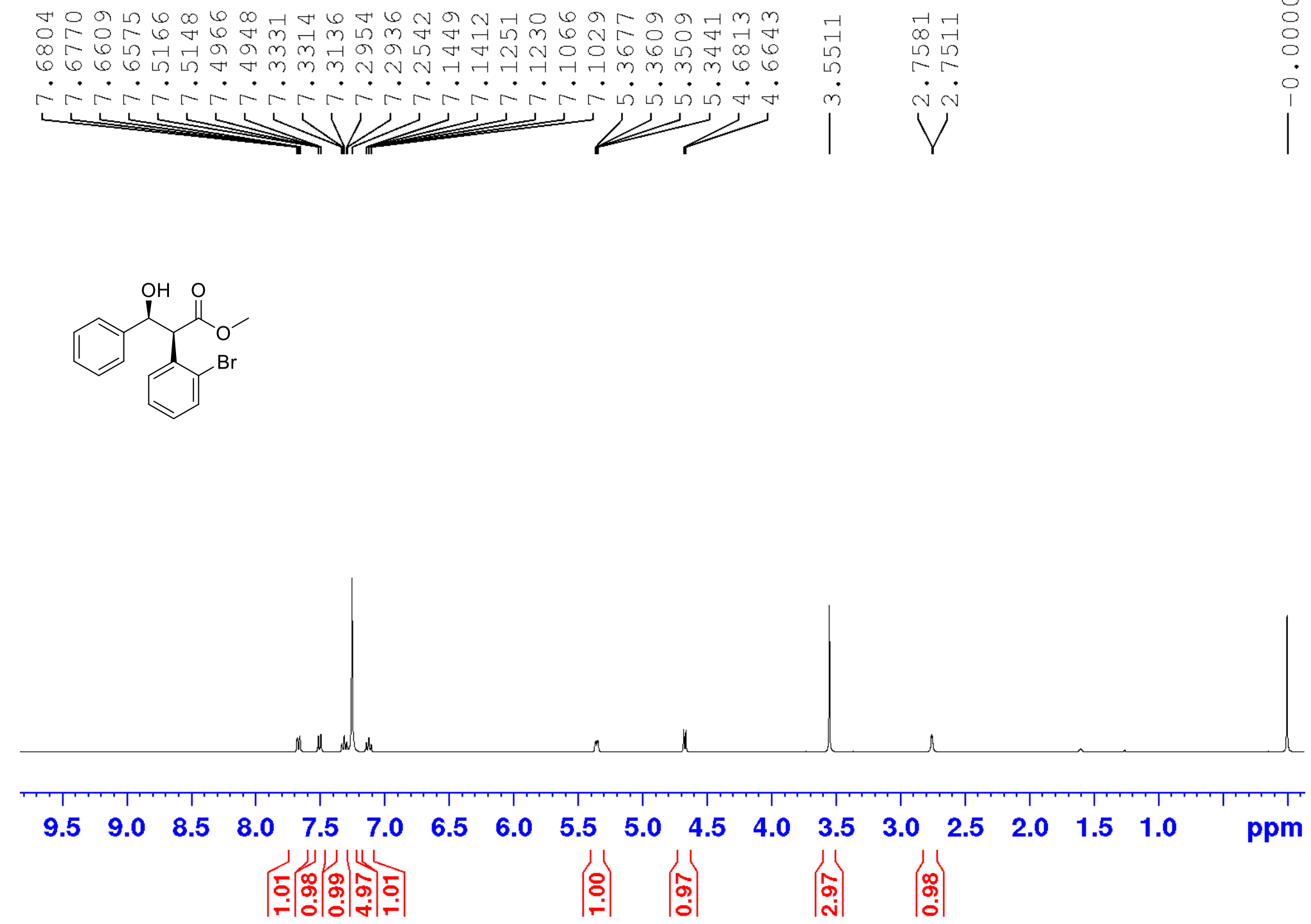

${ }^{1} \mathrm{H}$ NMR (400 MHz, $\mathrm{CDCl}_{3}$ ) Spectrum of syn-methyl 2-(2-bromophenyl)-3-hydroxy-3-phenylpropanoate (10h) 

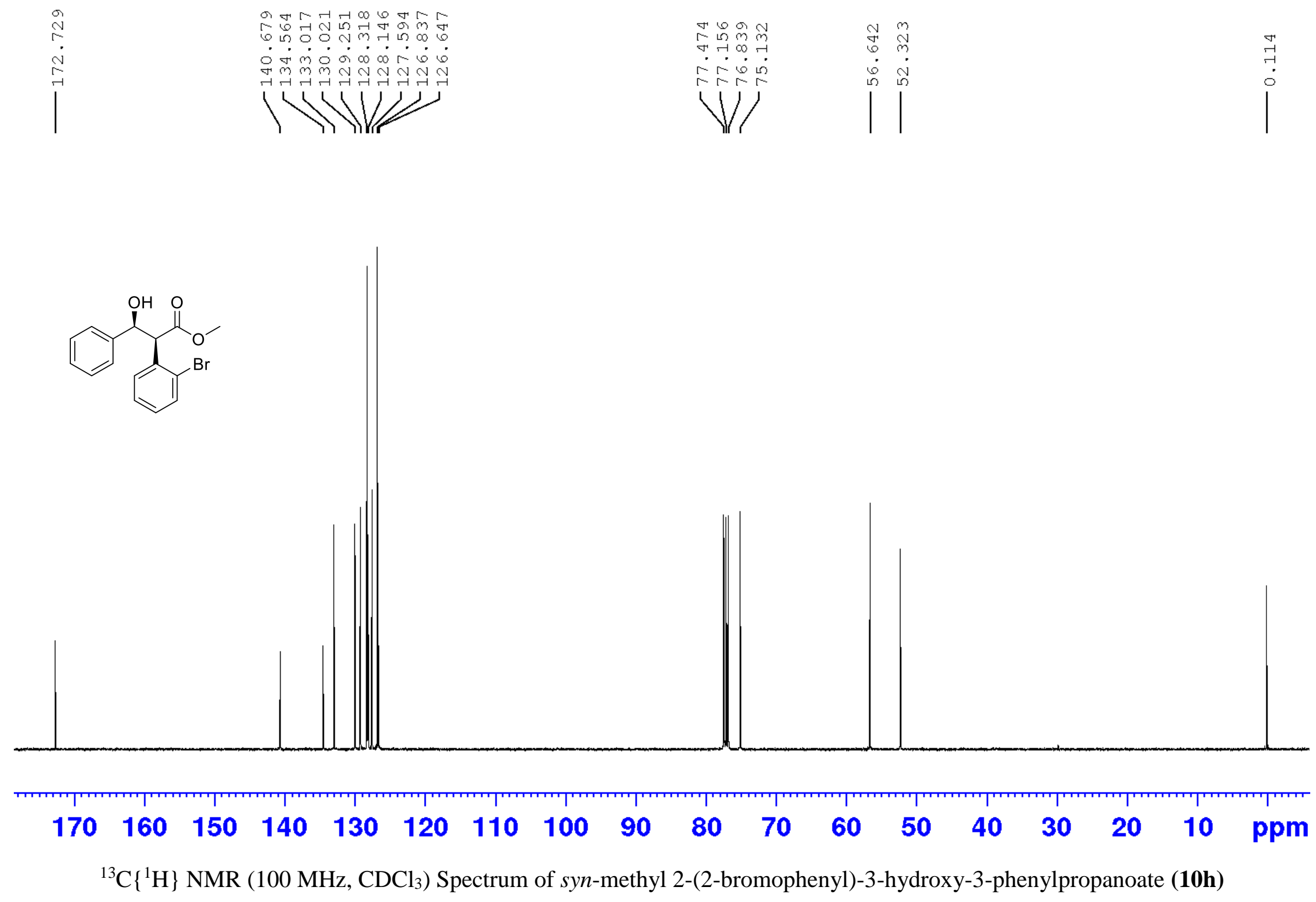

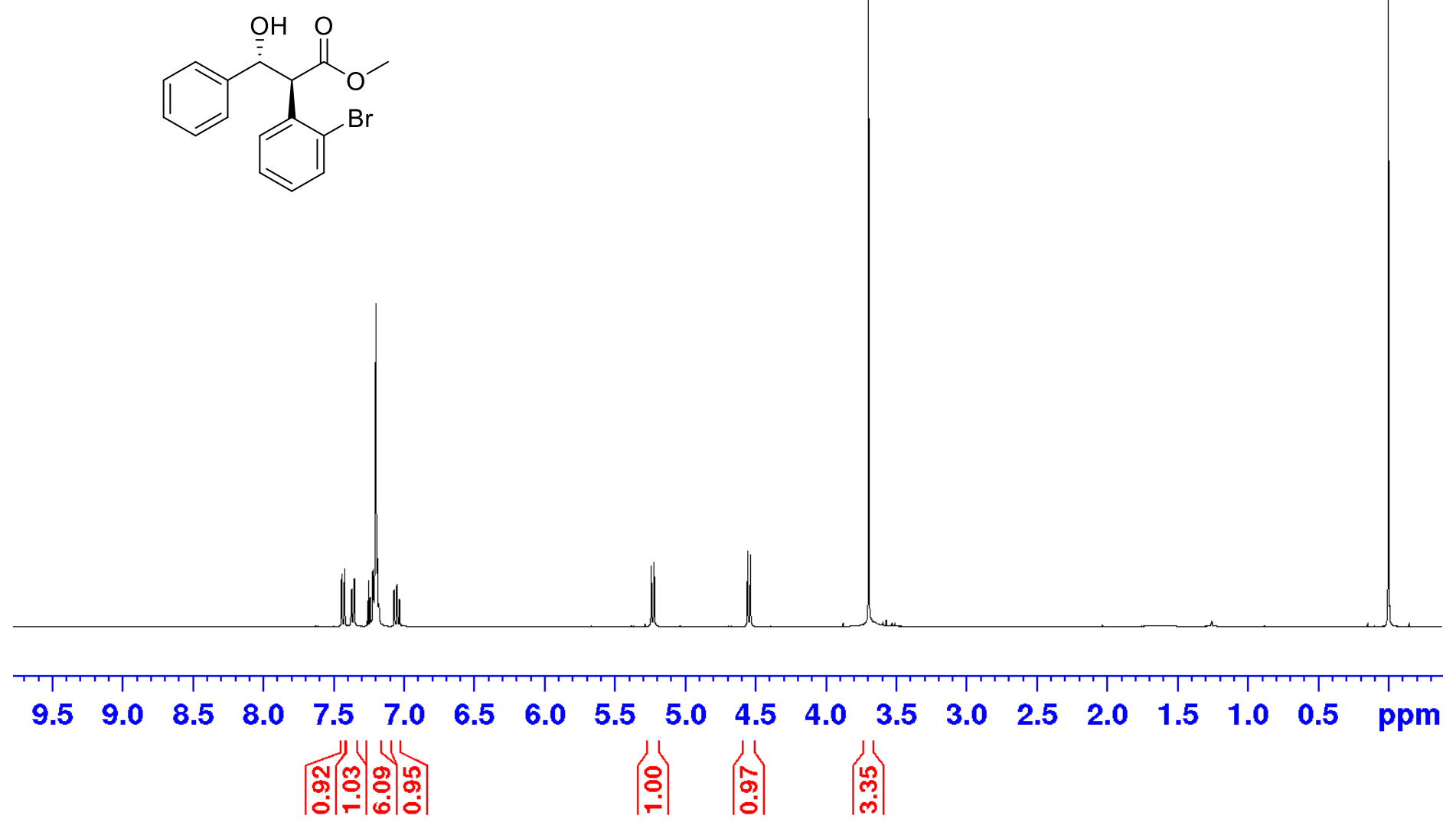

${ }^{1} \mathrm{H}$ NMR (400 MHz, $\mathrm{CDCl}_{3}$ ) Spectrum of anti-methyl 2-(2-bromophenyl)-3-hydroxy-3-phenylpropanoate (10h) 


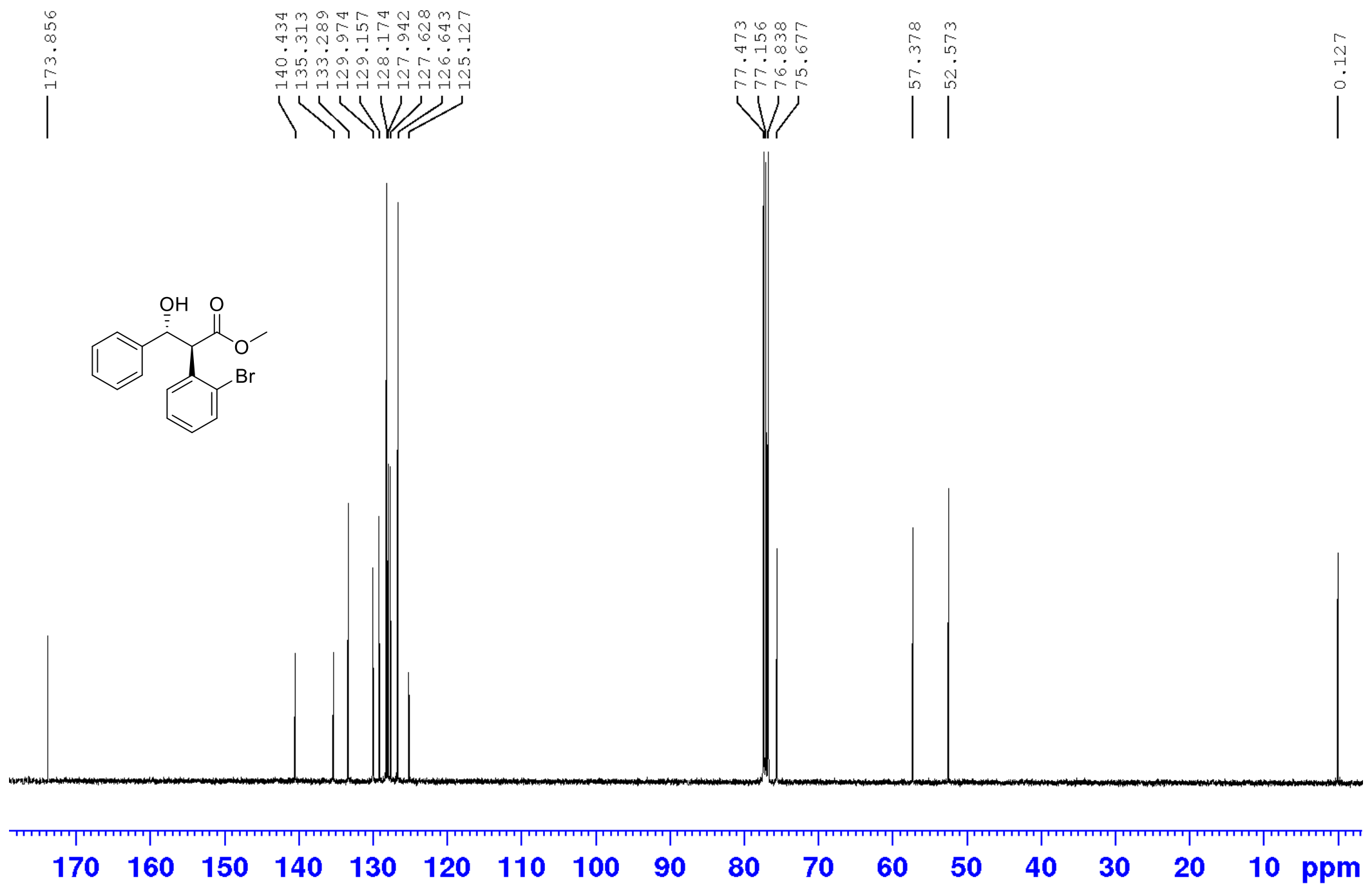

${ }^{13} \mathrm{C}\left\{{ }^{1} \mathrm{H}\right\} \mathrm{NMR}\left(100 \mathrm{MHz}, \mathrm{CDCl}_{3}\right)$ Spectrum of anti-methyl 2-(2-bromophenyl)-3-hydroxy-3-phenylpropanoate (10h) 


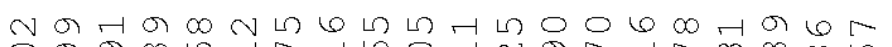

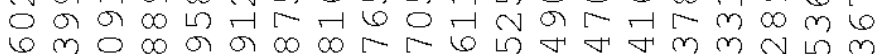

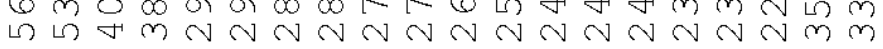

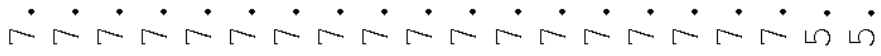
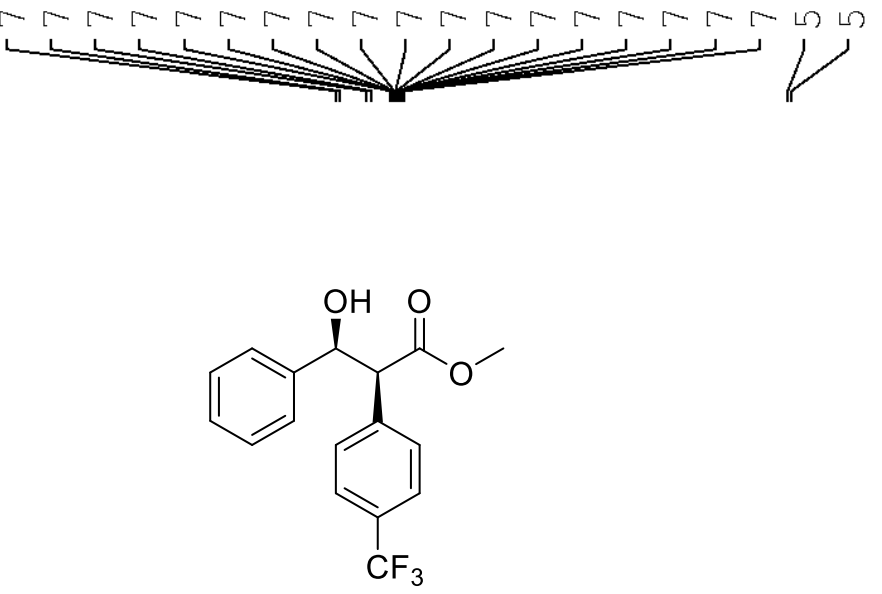
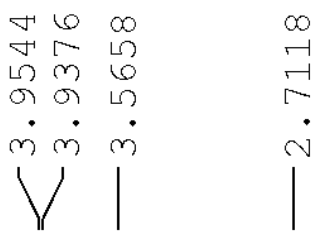
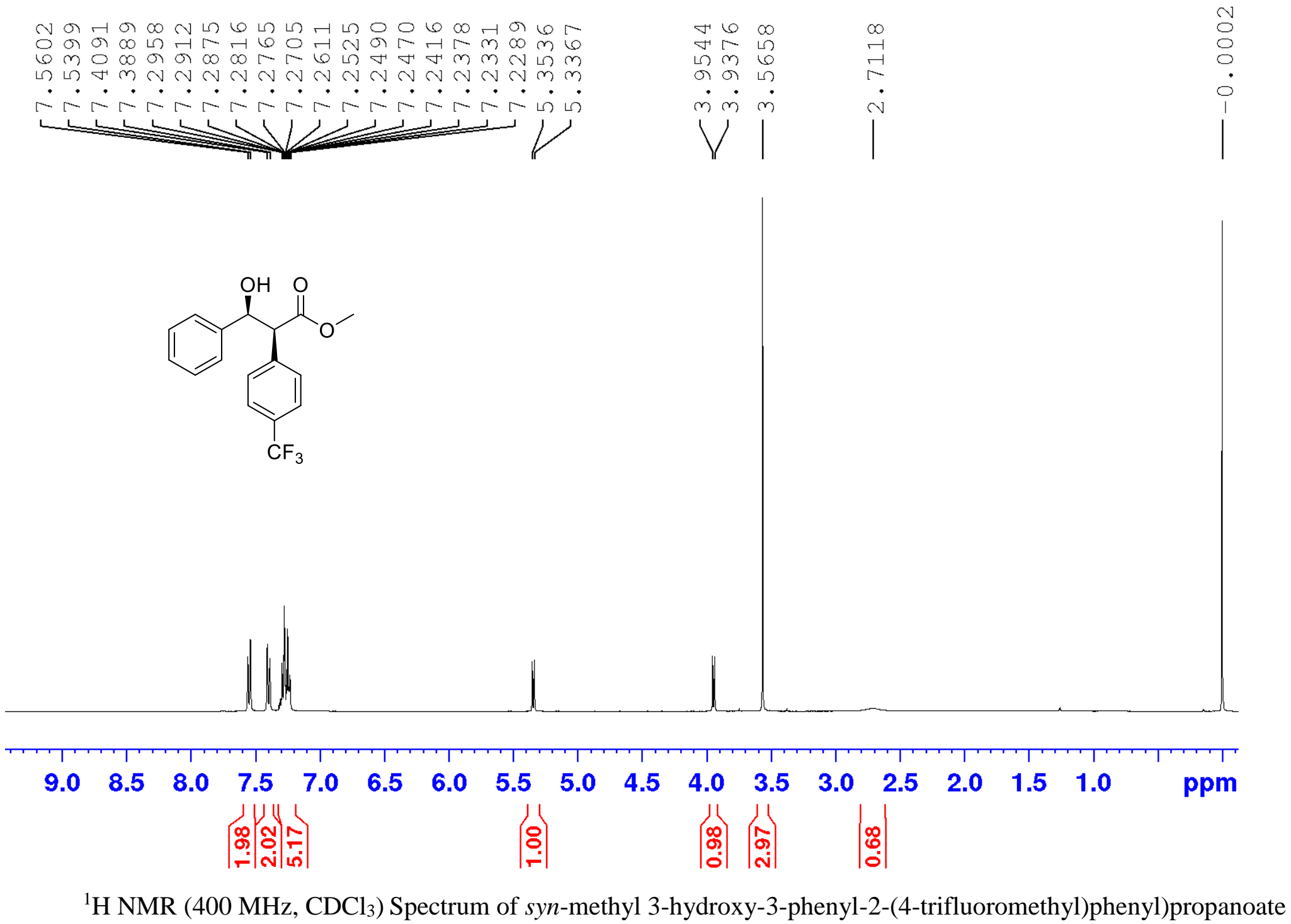

${ }^{1} \mathrm{H}$ NMR (400 MHz, $\mathrm{CDCl}_{3}$ ) Spectrum of syn-methyl 3-hydroxy-3-phenyl-2-(4-trifluoromethyl)phenyl)propanoate (10i)

S74 
$\left.\right|_{\substack{n \\ n}} ^{n}$
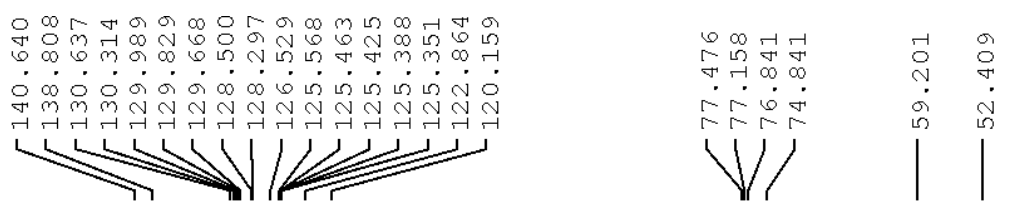

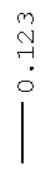
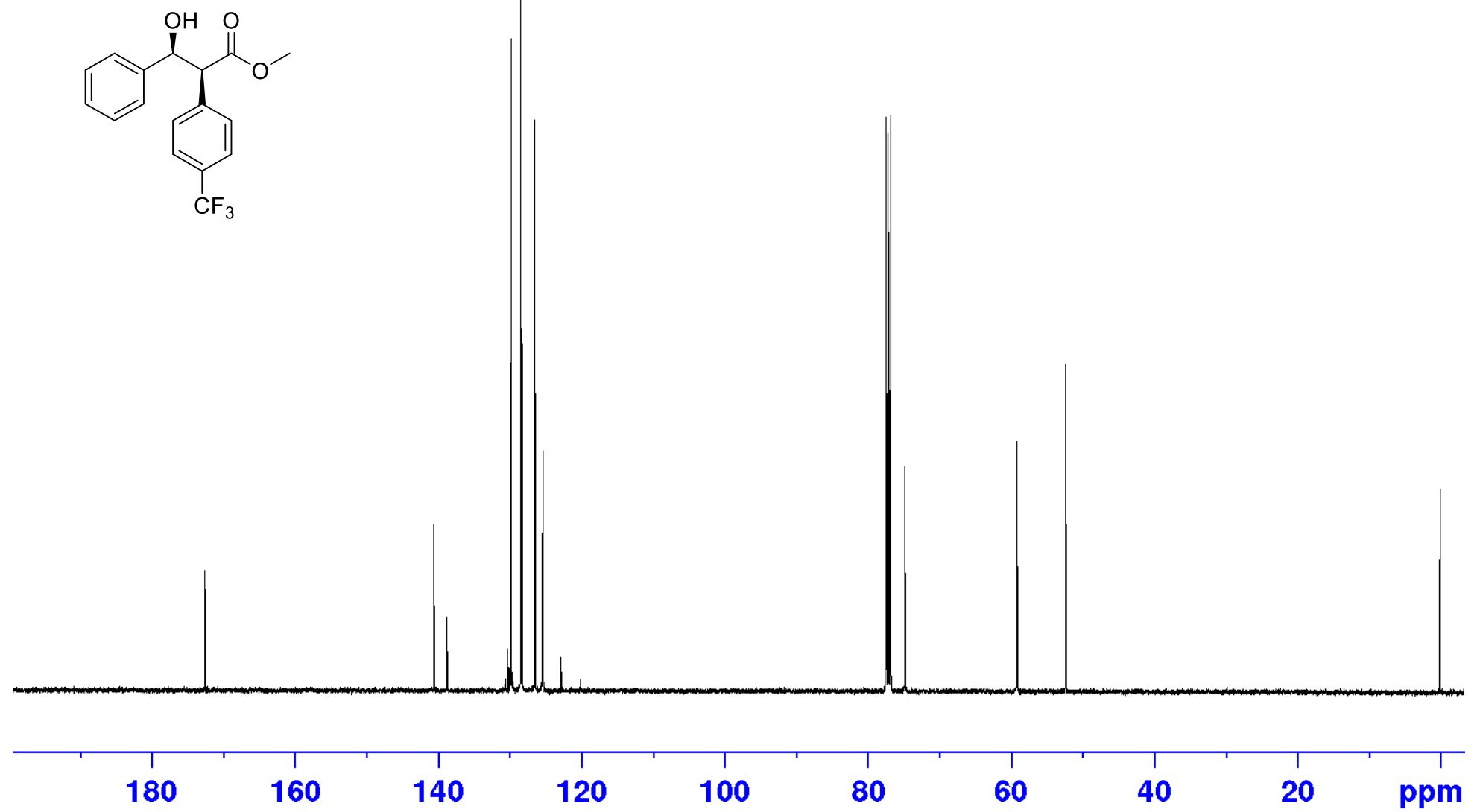

${ }^{13} \mathrm{C}\left\{{ }^{1} \mathrm{H}\right\} \mathrm{NMR}\left(100 \mathrm{MHz}, \mathrm{CDCl}_{3}\right)$ Spectrum of syn-methyl 3-hydroxy-3-phenyl-2-(4-trifluoromethyl)phenyl)propanoate (10i)

S75 

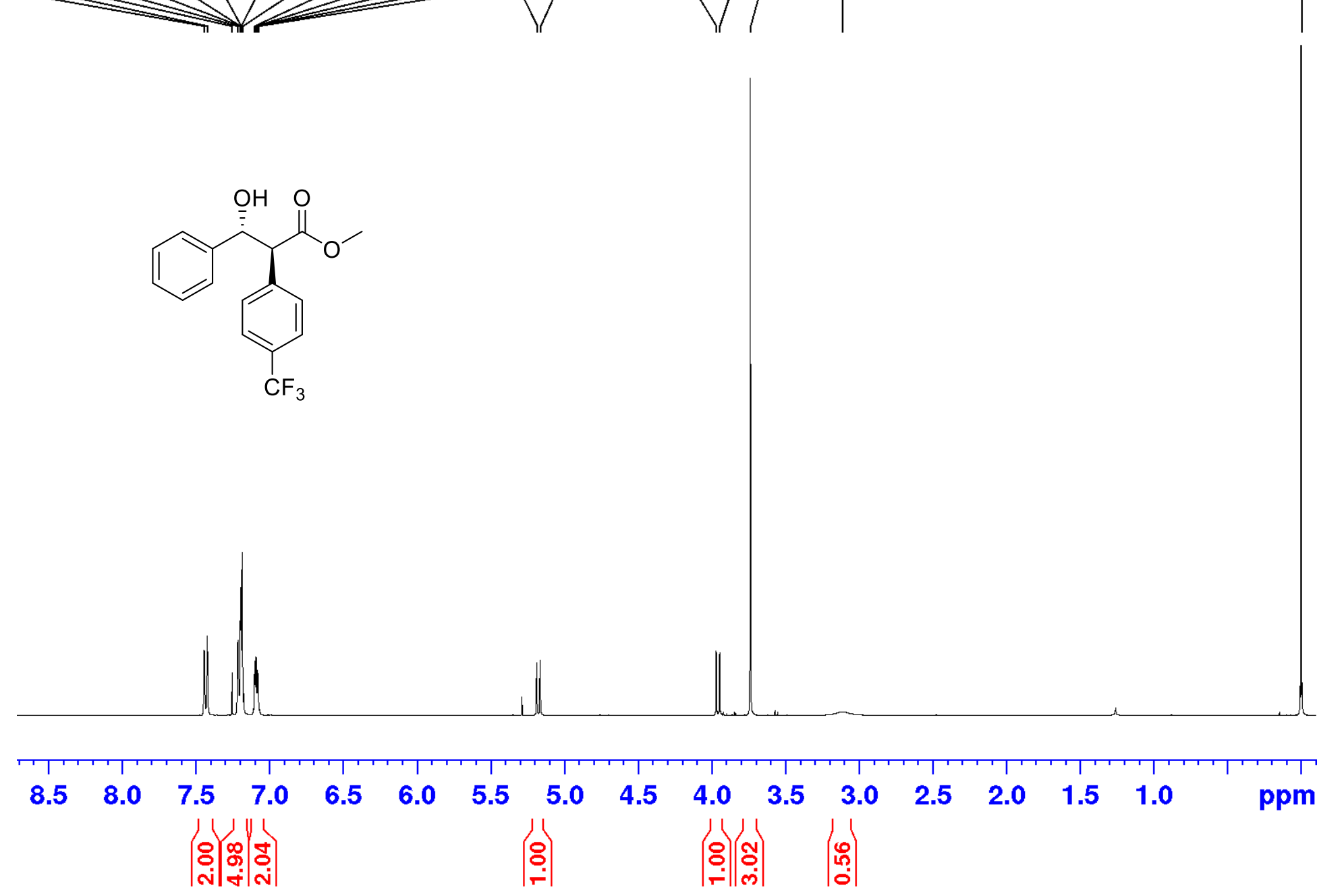

${ }^{1} \mathrm{H}$ NMR (400 MHz, $\mathrm{CDCl}_{3}$ ) Spectrum of anti-methyl 3-hydroxy-3-phenyl-2-(4-trifluoromethyl)phenyl)propanoate (10i) 

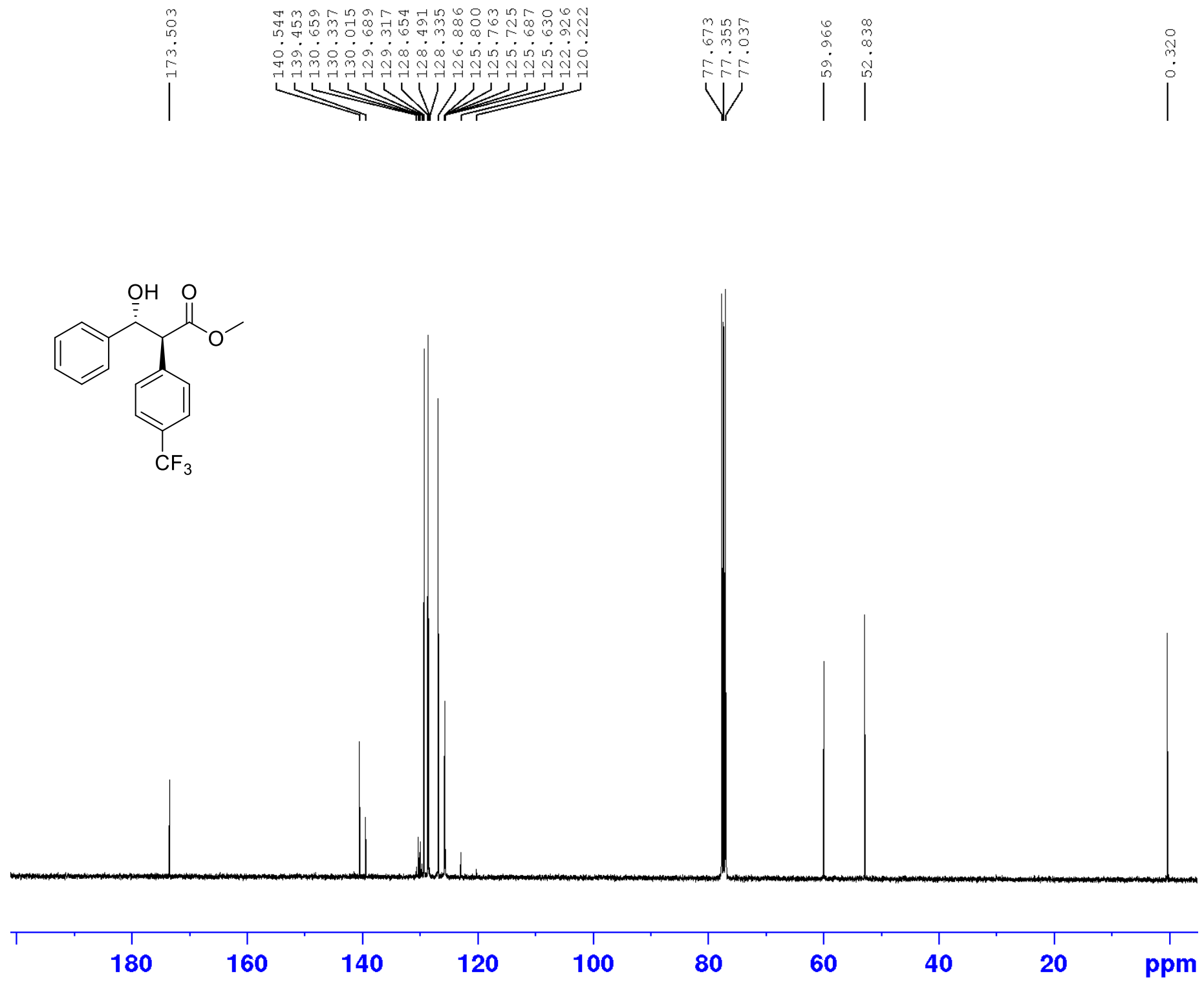

${ }^{13} \mathrm{C}\left\{{ }^{1} \mathrm{H}\right\}$ NMR (100 MHz, $\mathrm{CDCl}_{3}$ ) Spectrum of anti-methyl 3-hydroxy-3-phenyl-2-(4-trifluoromethyl)phenyl)propanoate (10i) 


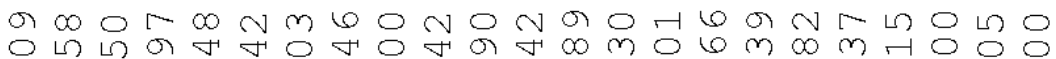
- 6 क म

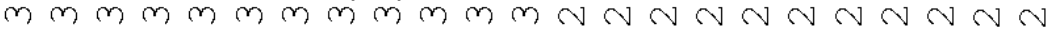

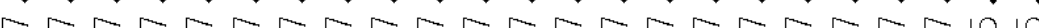
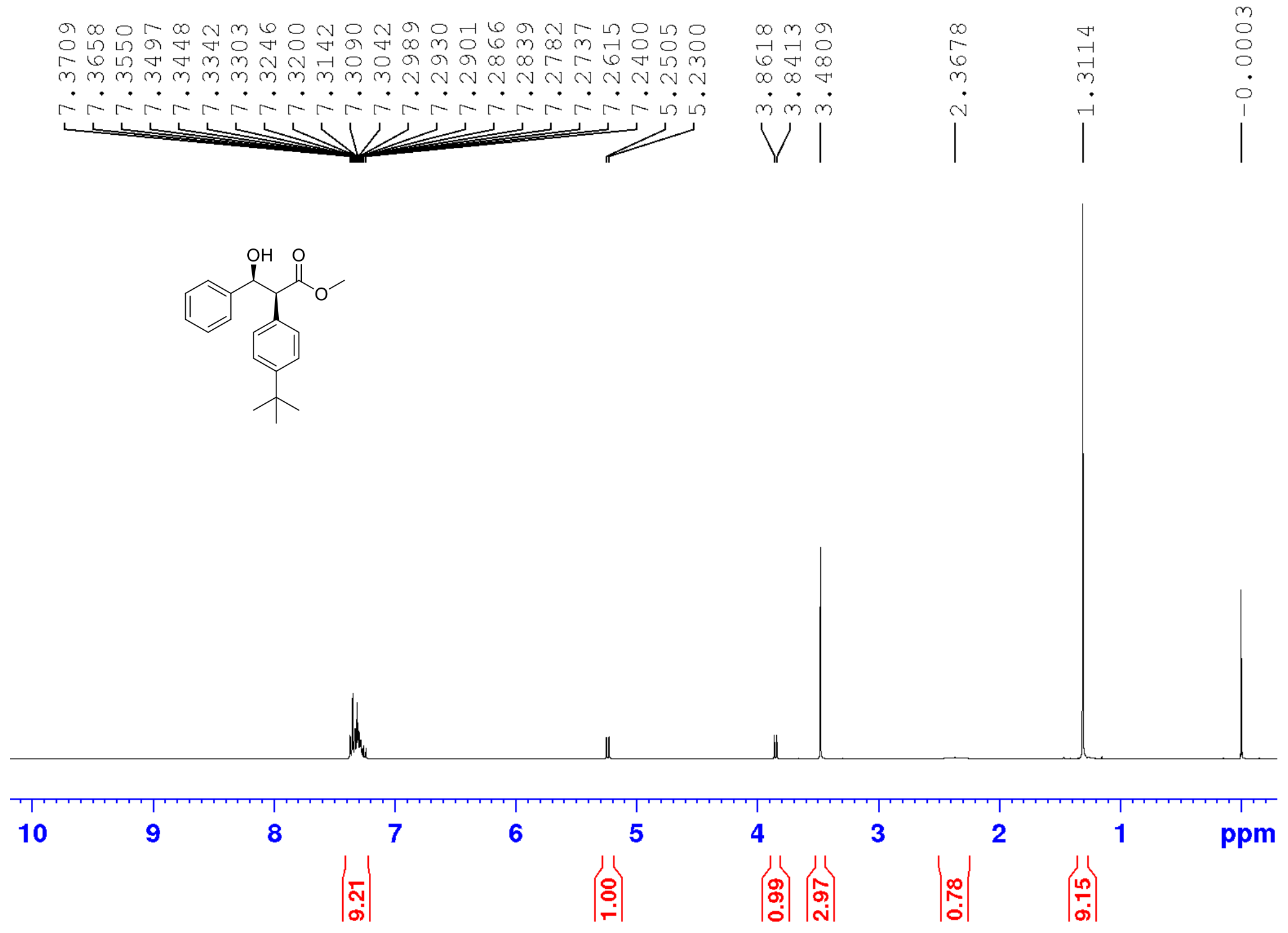

${ }^{1} \mathrm{H} \mathrm{NMR}\left(400 \mathrm{MHz}, \mathrm{CDCl}_{3}\right.$ ) Spectrum of syn-methyl 2-(4-tert-butylphenyl)-3-hydroxy-3-phenylpropanoate (10j) 

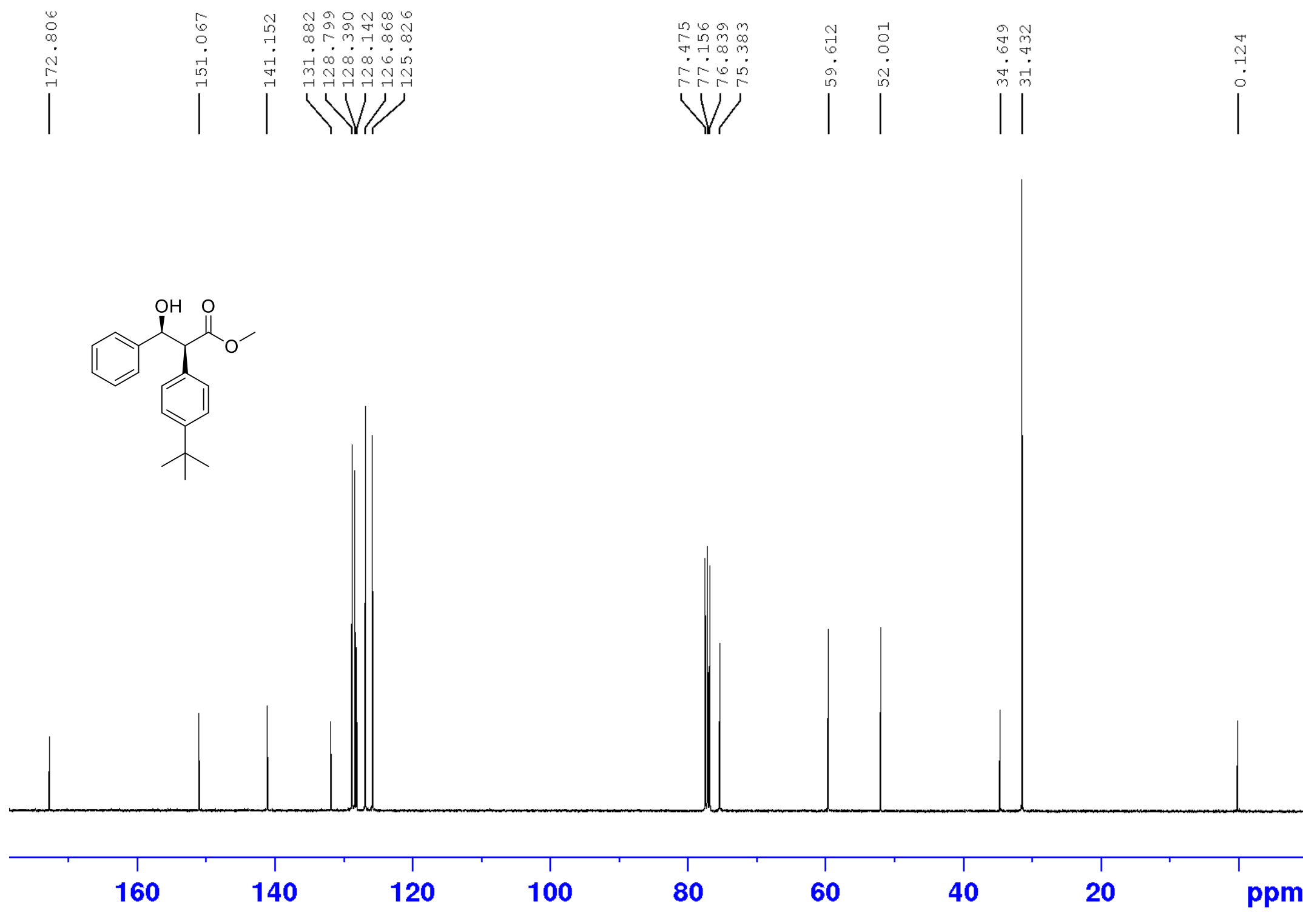

${ }^{13} \mathrm{C}\left\{{ }^{1} \mathrm{H}\right\}$ NMR (100 MHz, $\left.\mathrm{CDCl}_{3}\right)$ Spectrum of syn-methyl 2-(4-tert-butylphenyl)-3-hydroxy-3-phenylpropanoate (10j) 


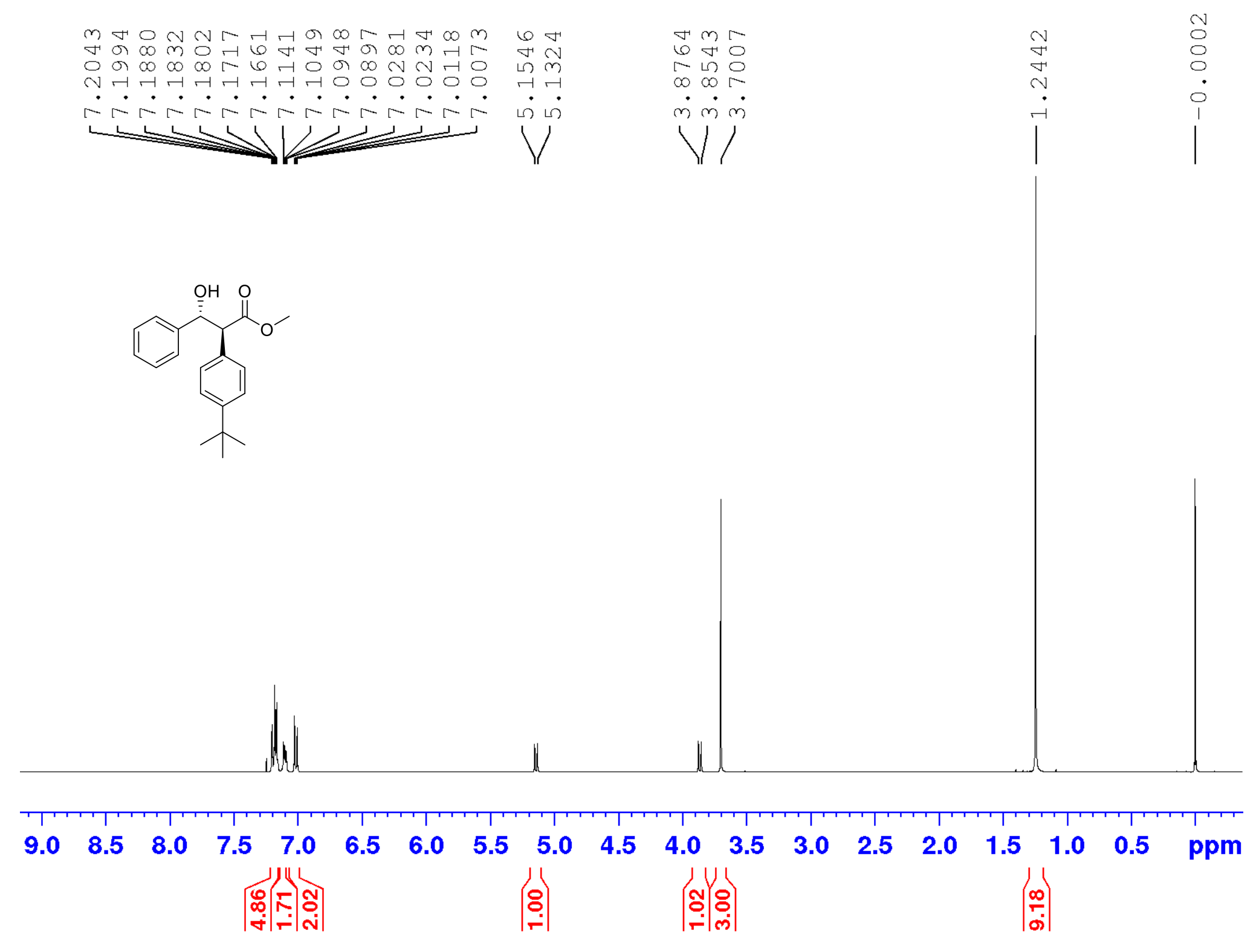

${ }^{1} \mathrm{H}$ NMR (400 MHz, $\mathrm{CDCl}_{3}$ ) Spectrum of anti-methyl 2-(4-tert-butylphenyl)-3-hydroxy-3-phenylpropanoate (10j) 


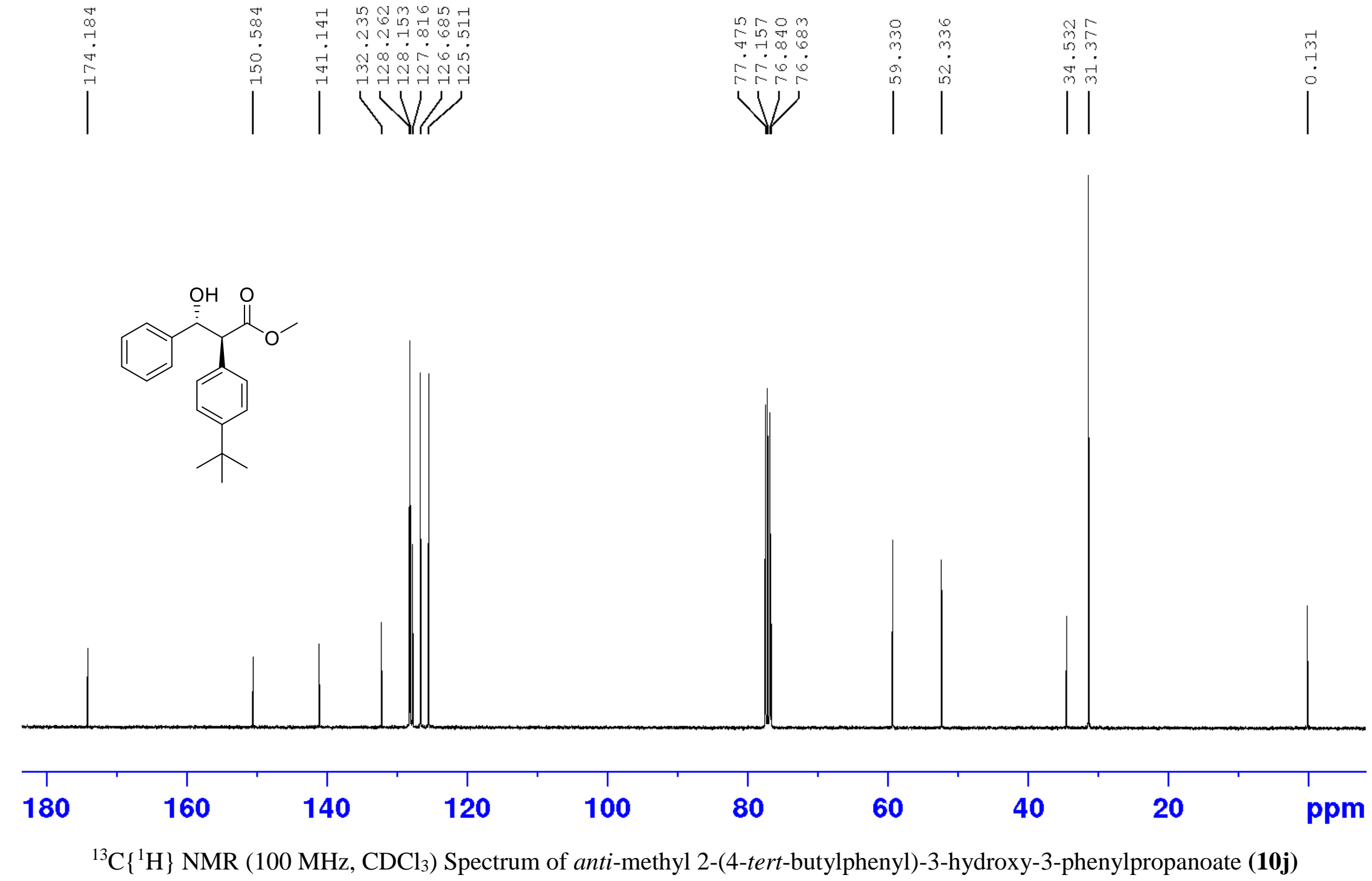


の

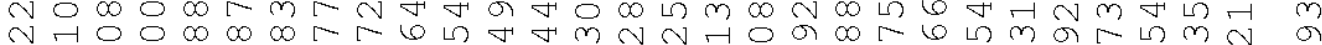

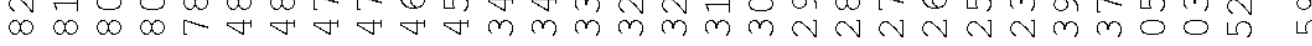

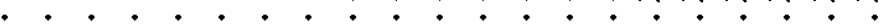

$L=$

1

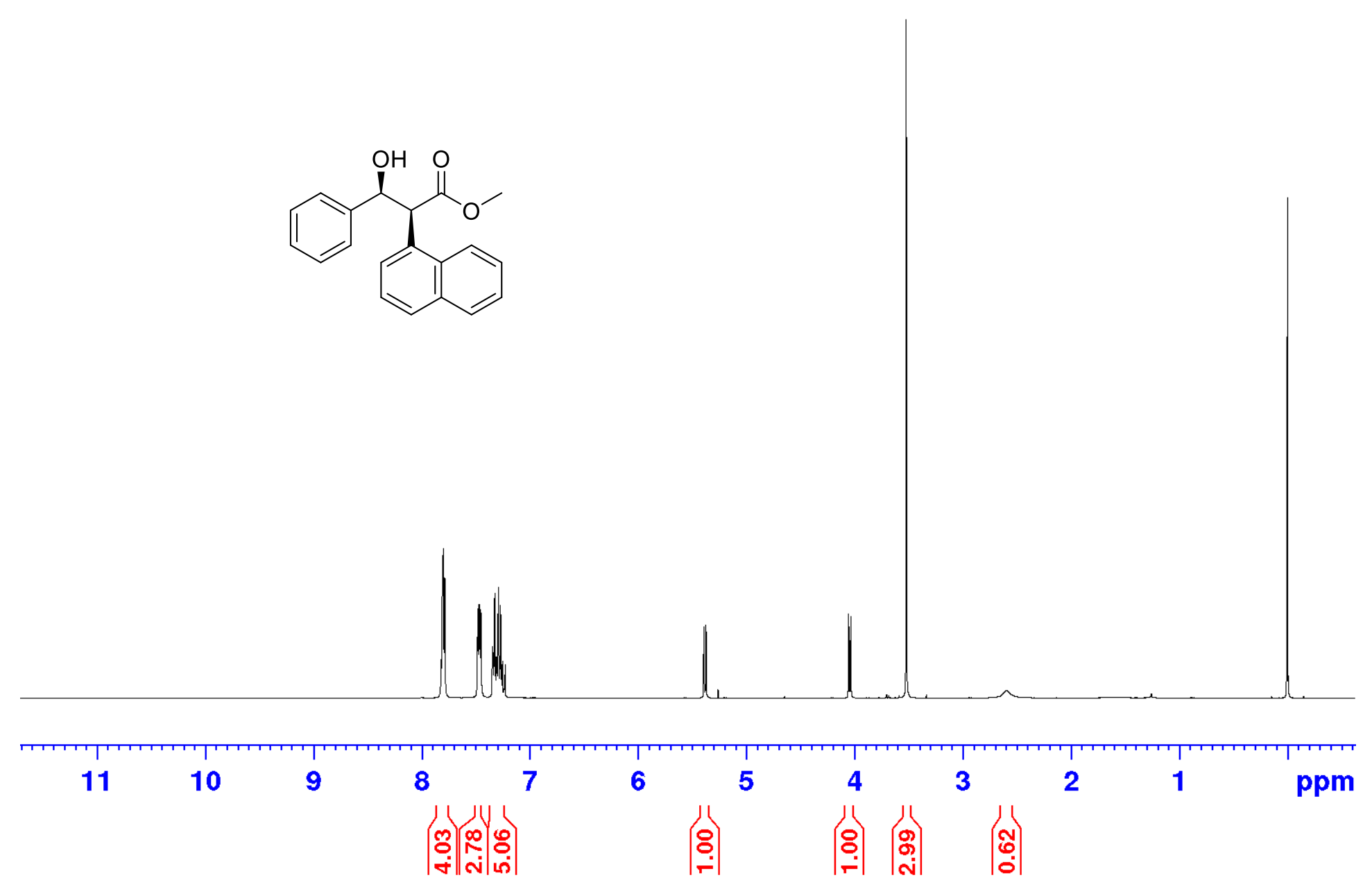

${ }^{1} \mathrm{H}$ NMR (400 MHz, $\mathrm{CDCl}_{3}$ ) Spectrum of syn-methyl 3-hydroxy-2-(naphthalen-1-yl)-3-phenylpropanoate (10k) 


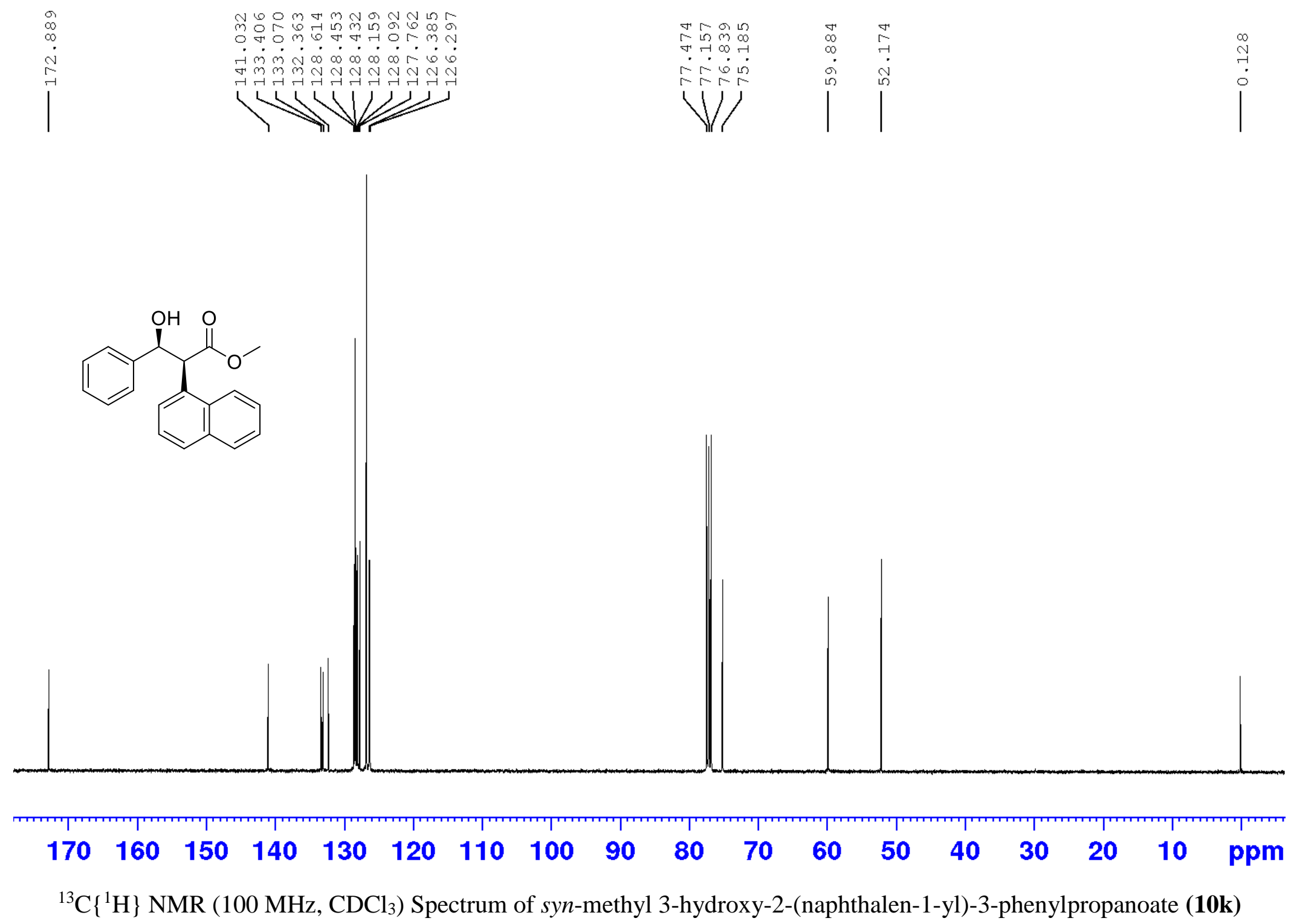


[ 小 小mN

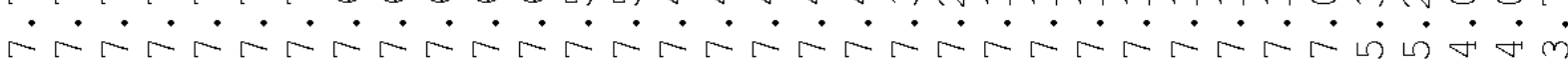
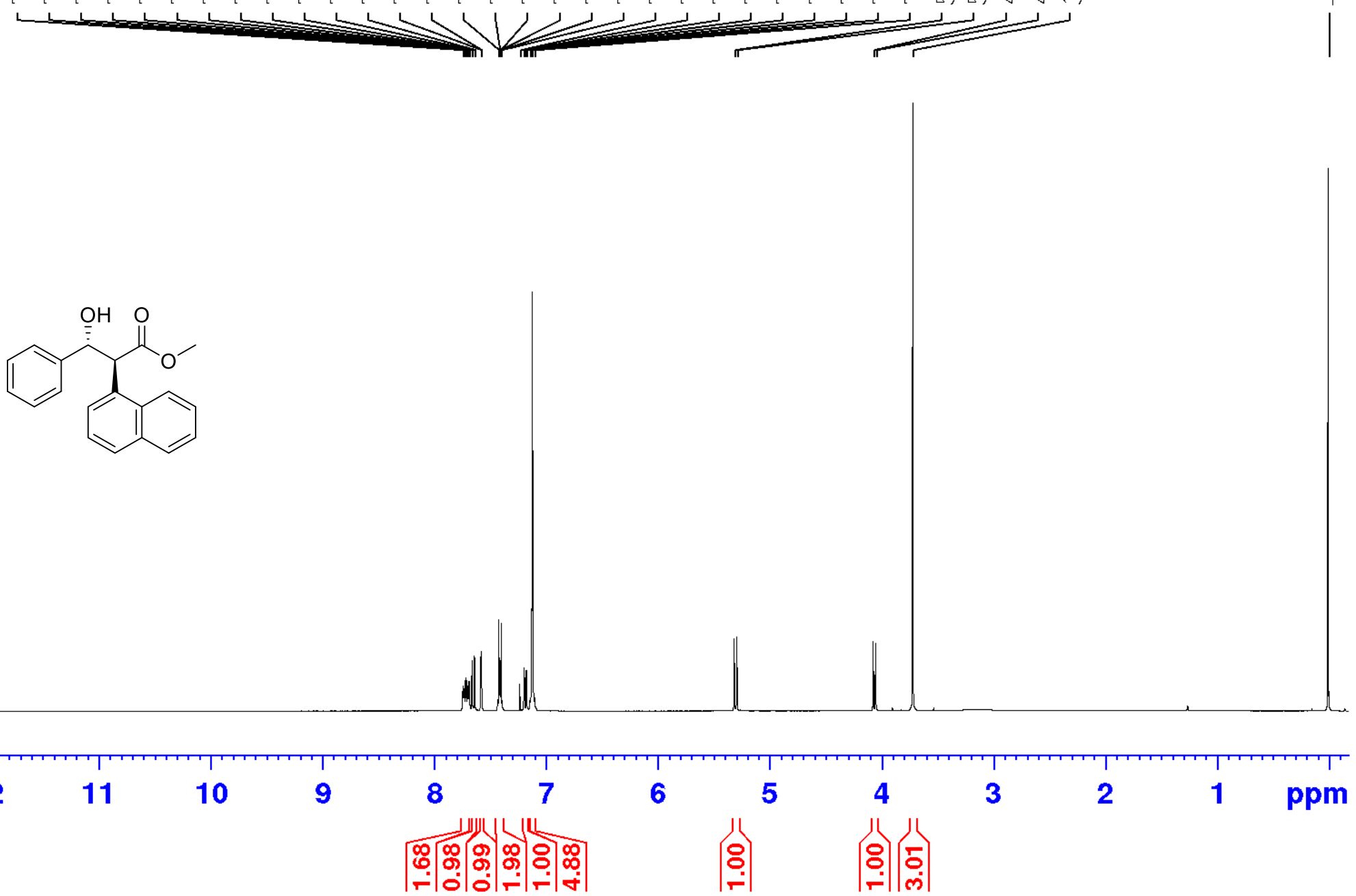

${ }^{1} \mathrm{H}$ NMR (400 MHz, $\mathrm{CDCl}_{3}$ ) Spectrum of anti-methyl 3-hydroxy-2-(naphthalen-1-yl)-3-phenylpropanoate (10k) 

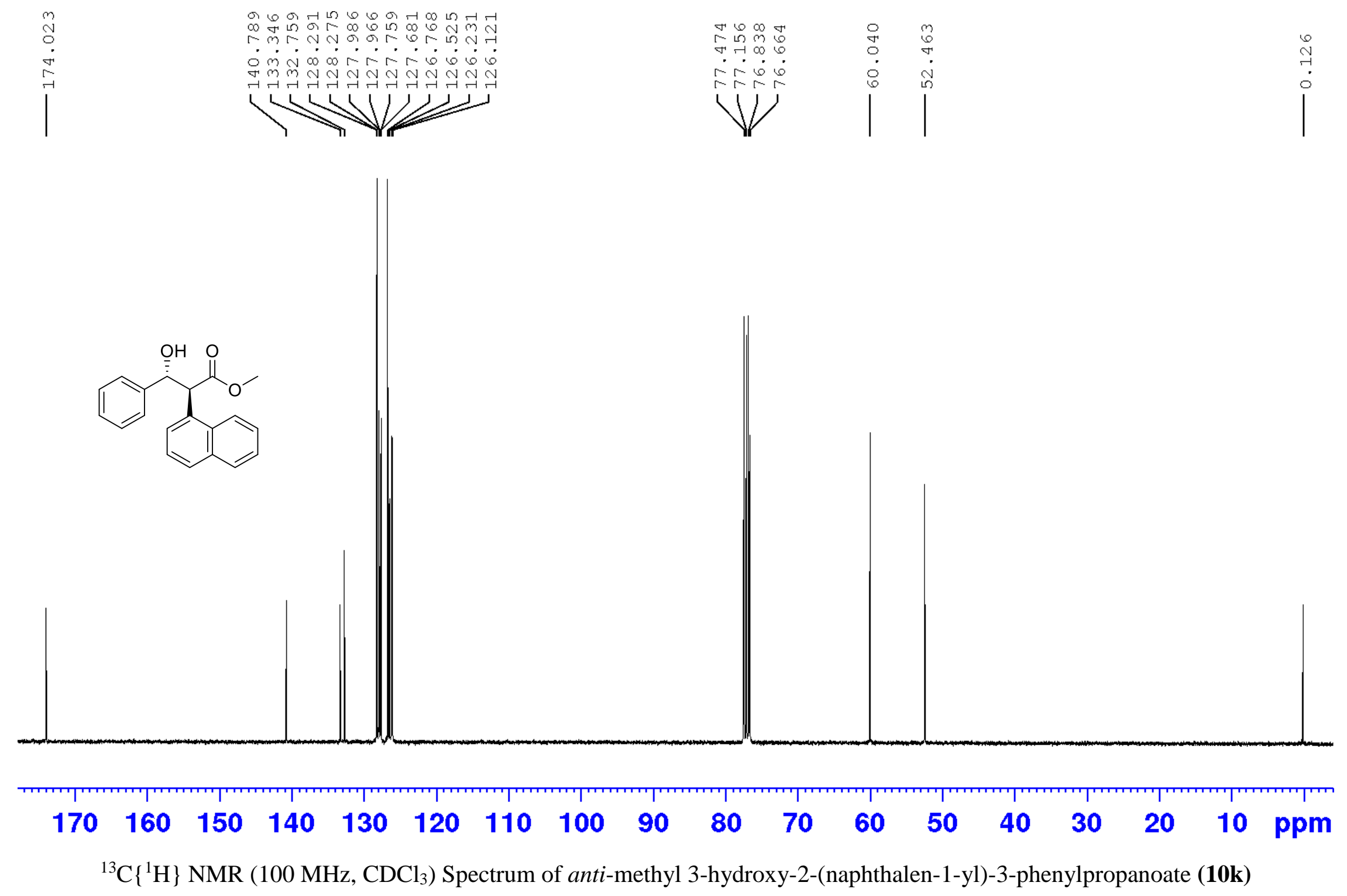

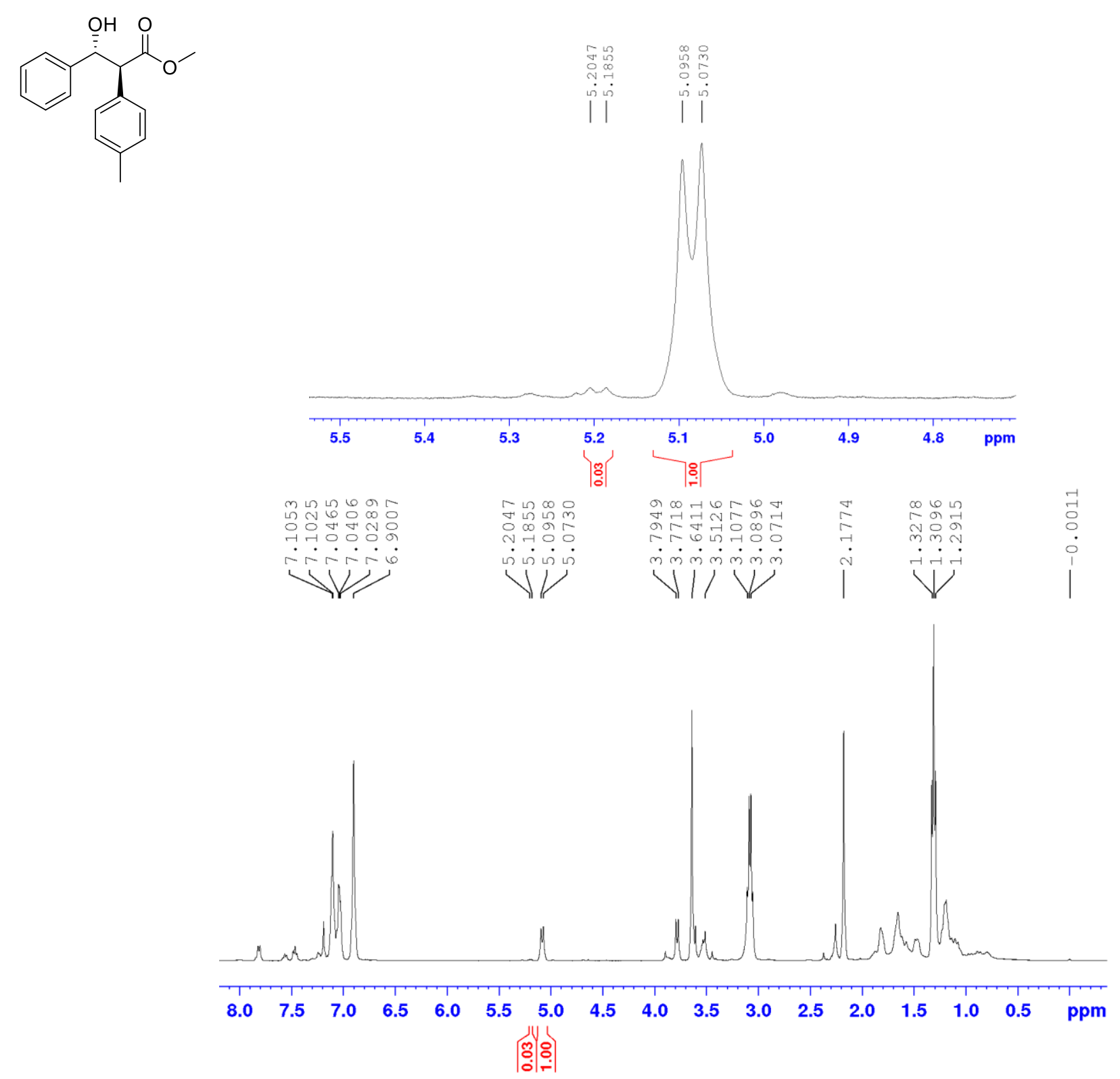

${ }^{1} \mathrm{H} \mathrm{NMR}\left(400 \mathrm{MHz}, \mathrm{CDCl}_{3}\right.$ ) Spectrum of crude anti-methyl 3-hydroxy-3-phenyl-2-p-tolylpropanoate 

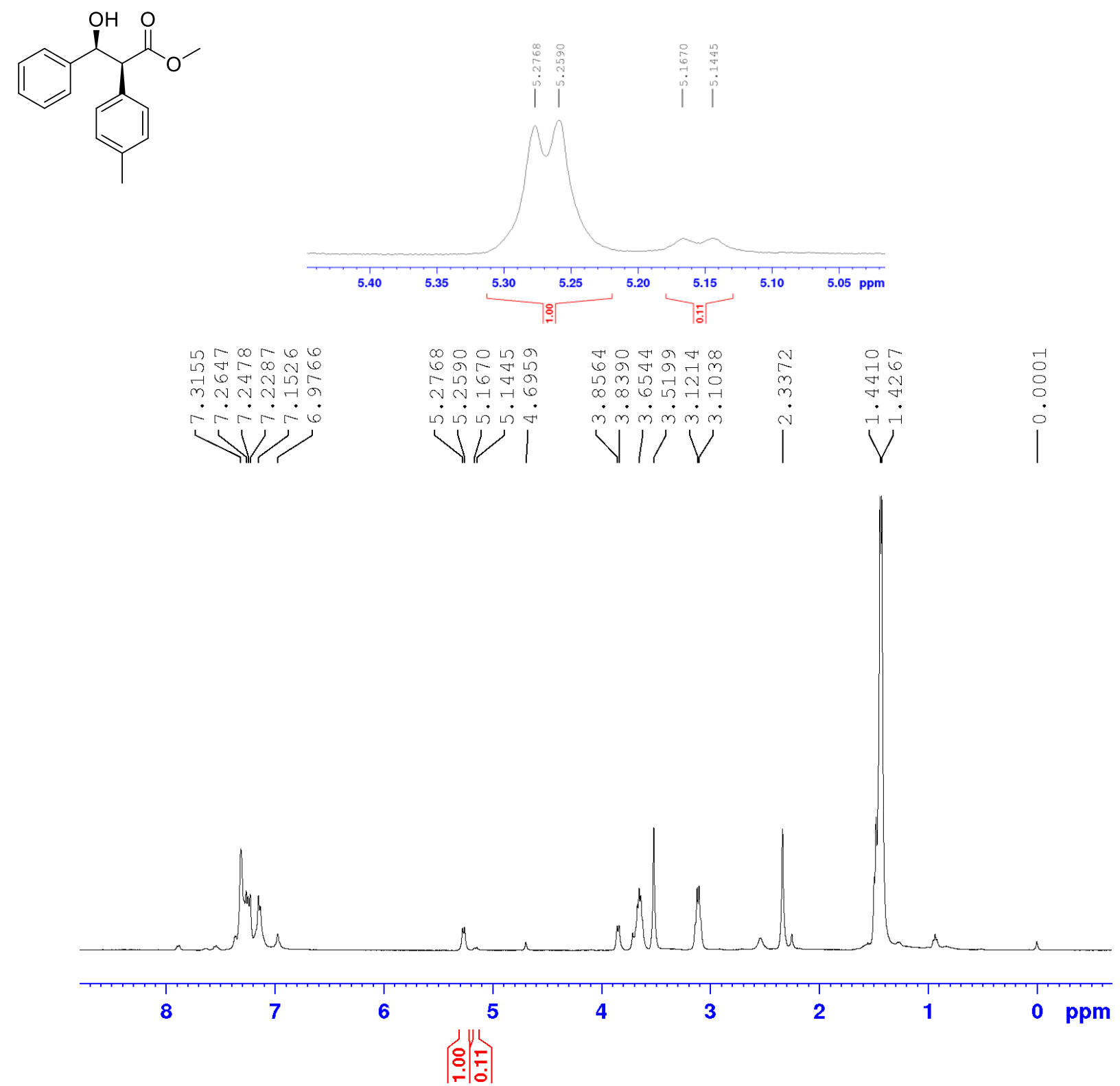

${ }^{1} \mathrm{H}$ NMR (400 MHz, $\mathrm{CDCl}_{3}$ ) Spectrum of crude syn-methyl 3-hydroxy-3-phenyl-2-p-tolylpropanoate 

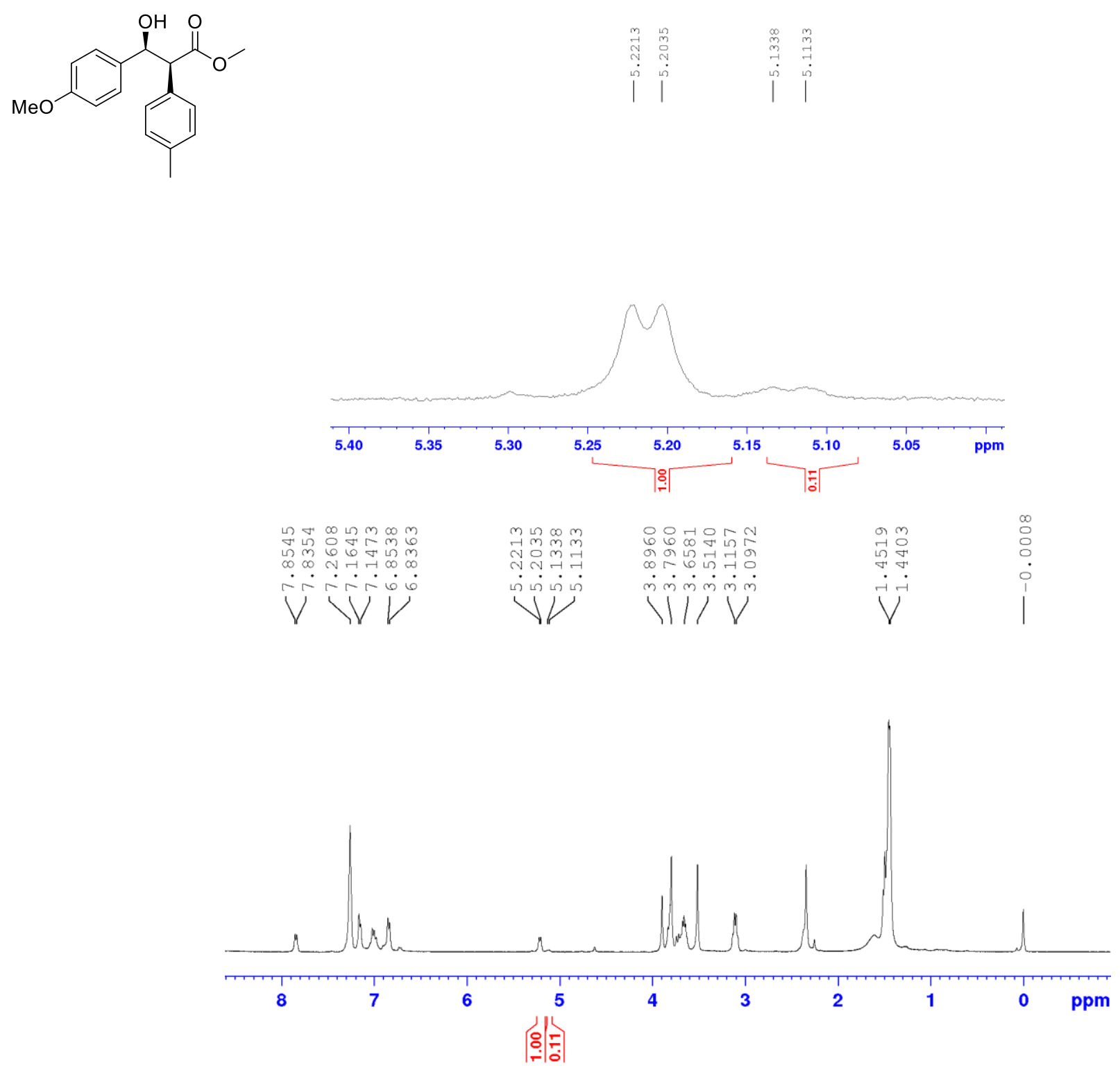

${ }^{1} \mathrm{H}$ NMR (400 MHz, $\mathrm{CDCl}_{3}$ ) Spectrum of crude syn-methyl 3-hydroxy-3-(4-methoxyphenyl)-2-p-tolylpropanoate 

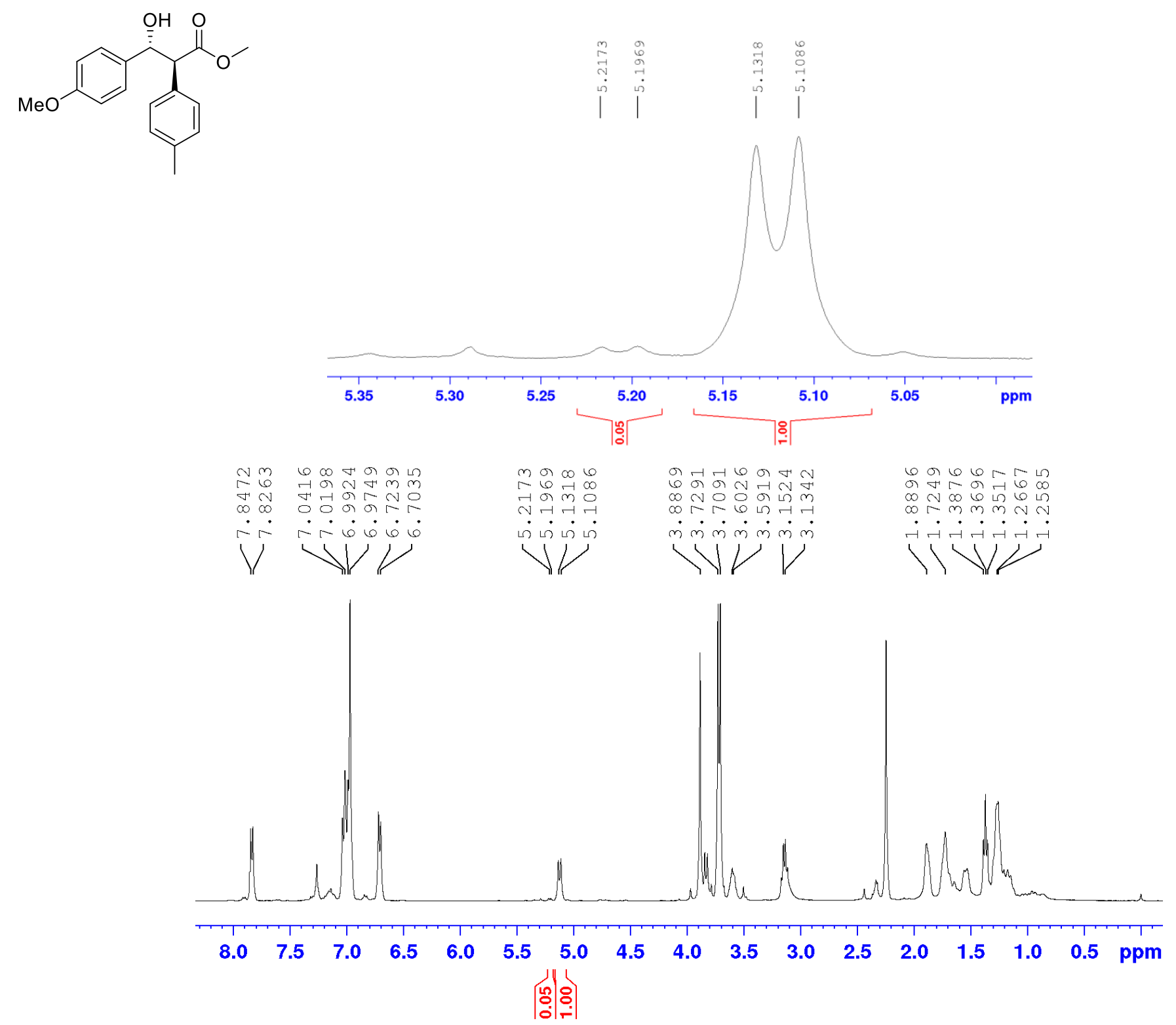

${ }^{1} \mathrm{H}$ NMR (400 MHz, $\mathrm{CDCl}_{3}$ ) Spectrum of crude anti-methyl 3-hydroxy-3-(4-methoxyphenyl)-2-p-tolylpropanoate 


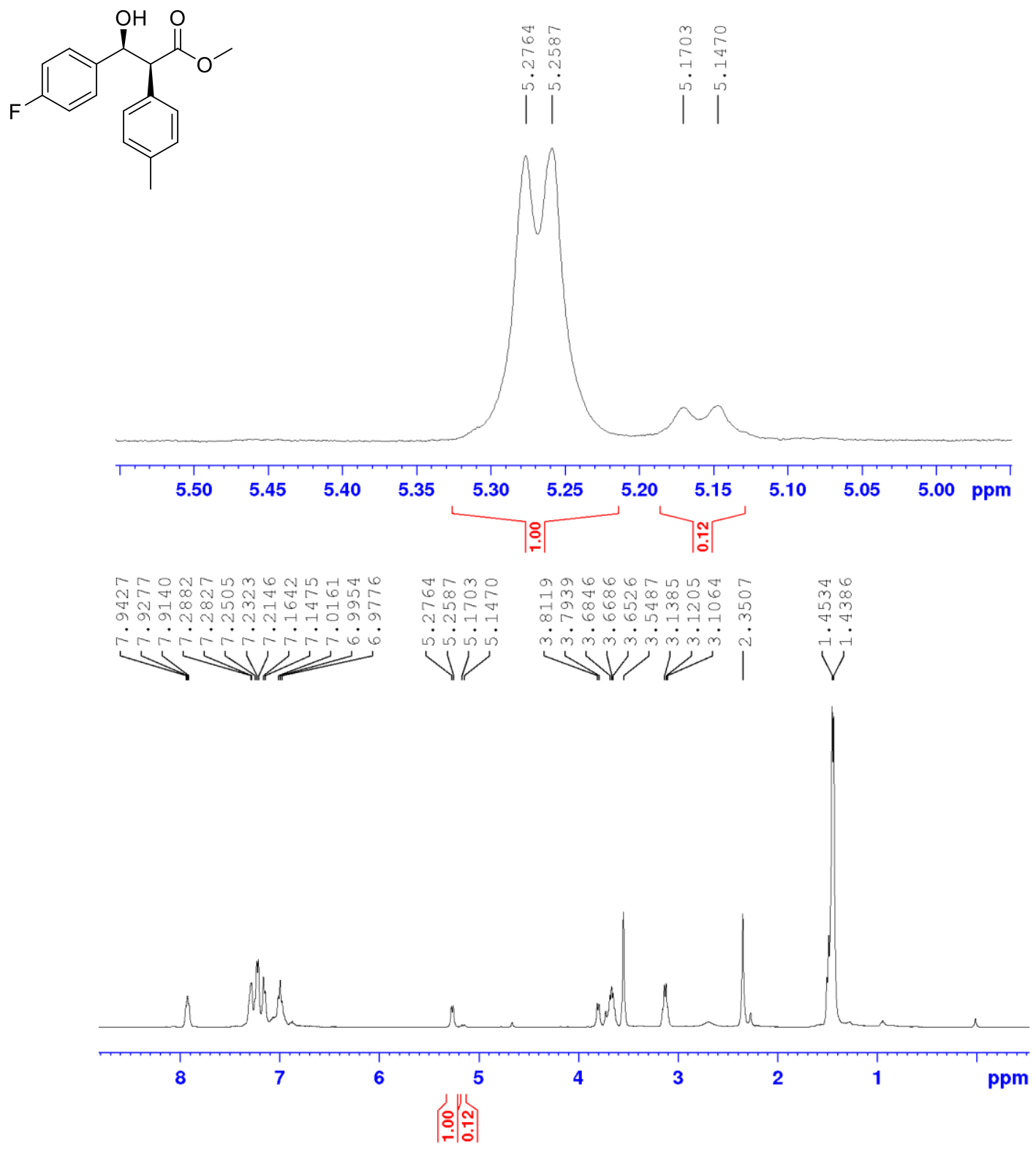

${ }^{1} \mathrm{H} \mathrm{NMR}\left(400 \mathrm{MHz}, \mathrm{CDCl}_{3}\right.$ ) Spectrum of crude syn-methyl 3-(4-fluorophenyl)-3-hydroxy-2-p-tolylpropanoate 

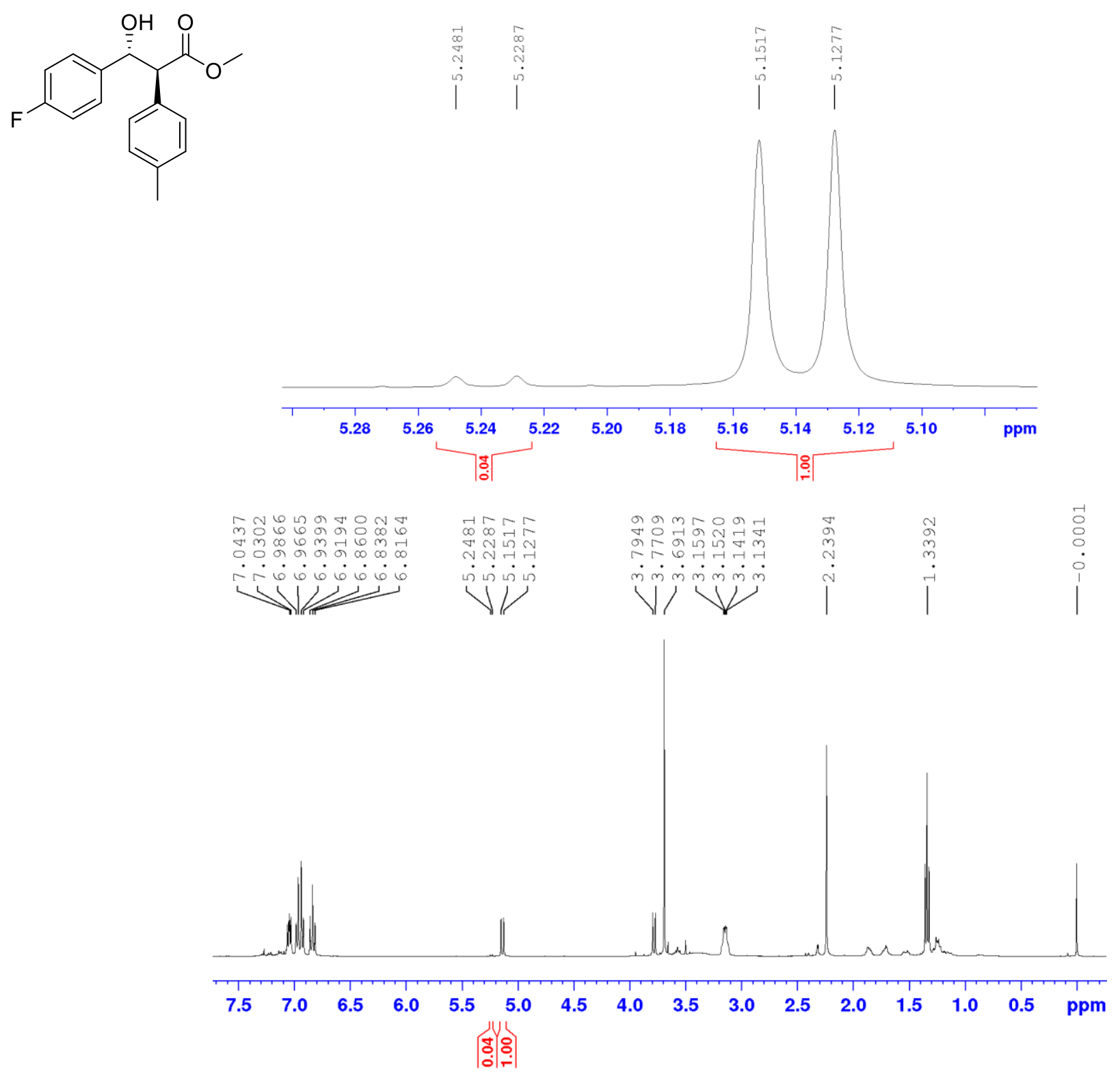

${ }^{1} \mathrm{H}$ NMR (400 MHz, $\mathrm{CDCl}_{3}$ ) Spectrum of crude anti-methyl 3-(4-fluorophenyl)-3-hydroxy-2-p-tolylpropanoate 

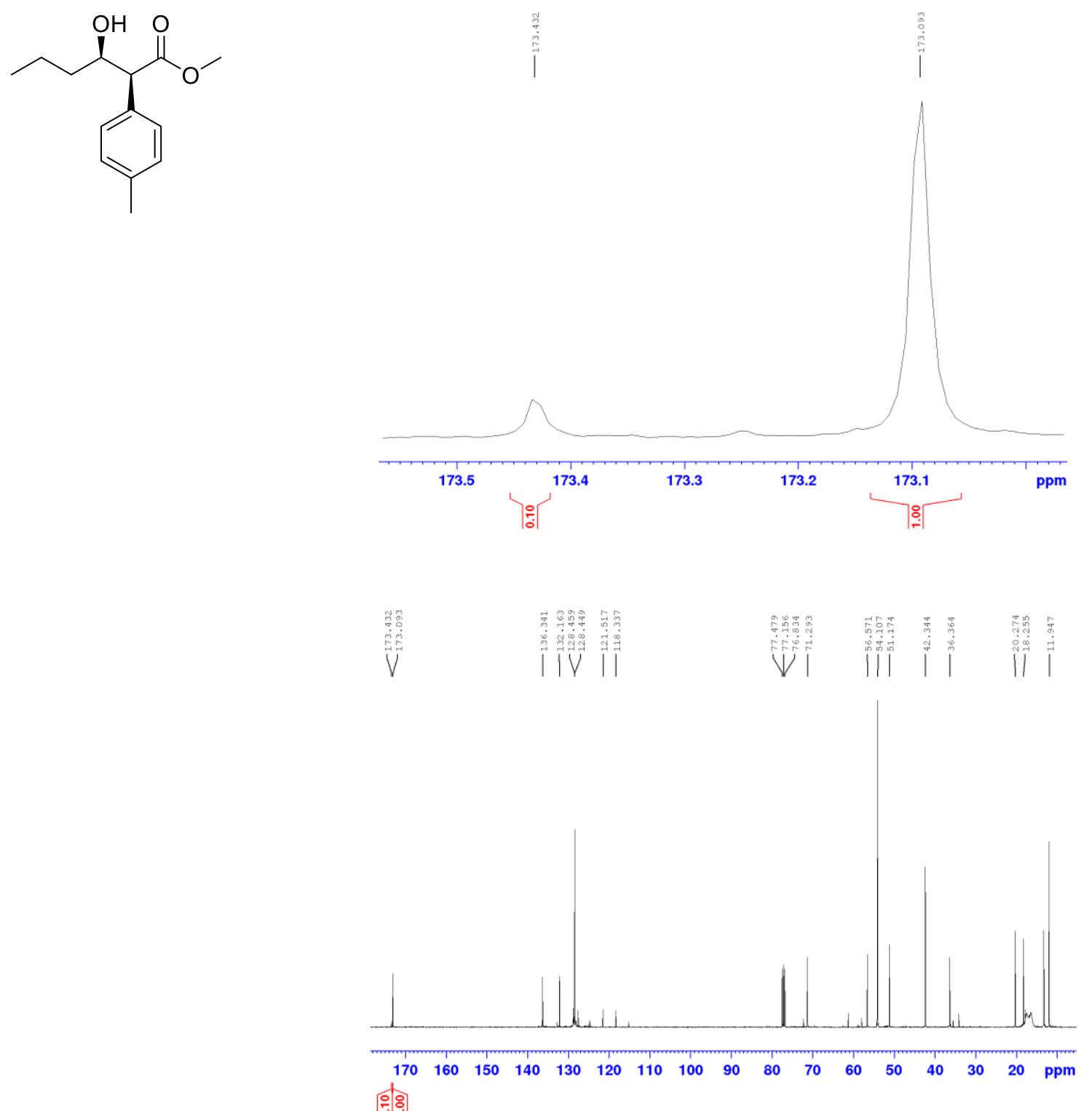

${ }^{13} \mathrm{C}\left\{{ }^{1} \mathrm{H}\right\}$ NMR $\left(100 \mathrm{MHz}, \mathrm{CDCl}_{3}\right)$ Spectrum of crude syn-methyl 3-hydroxy-2-p-tolylhexanoate (7j) 

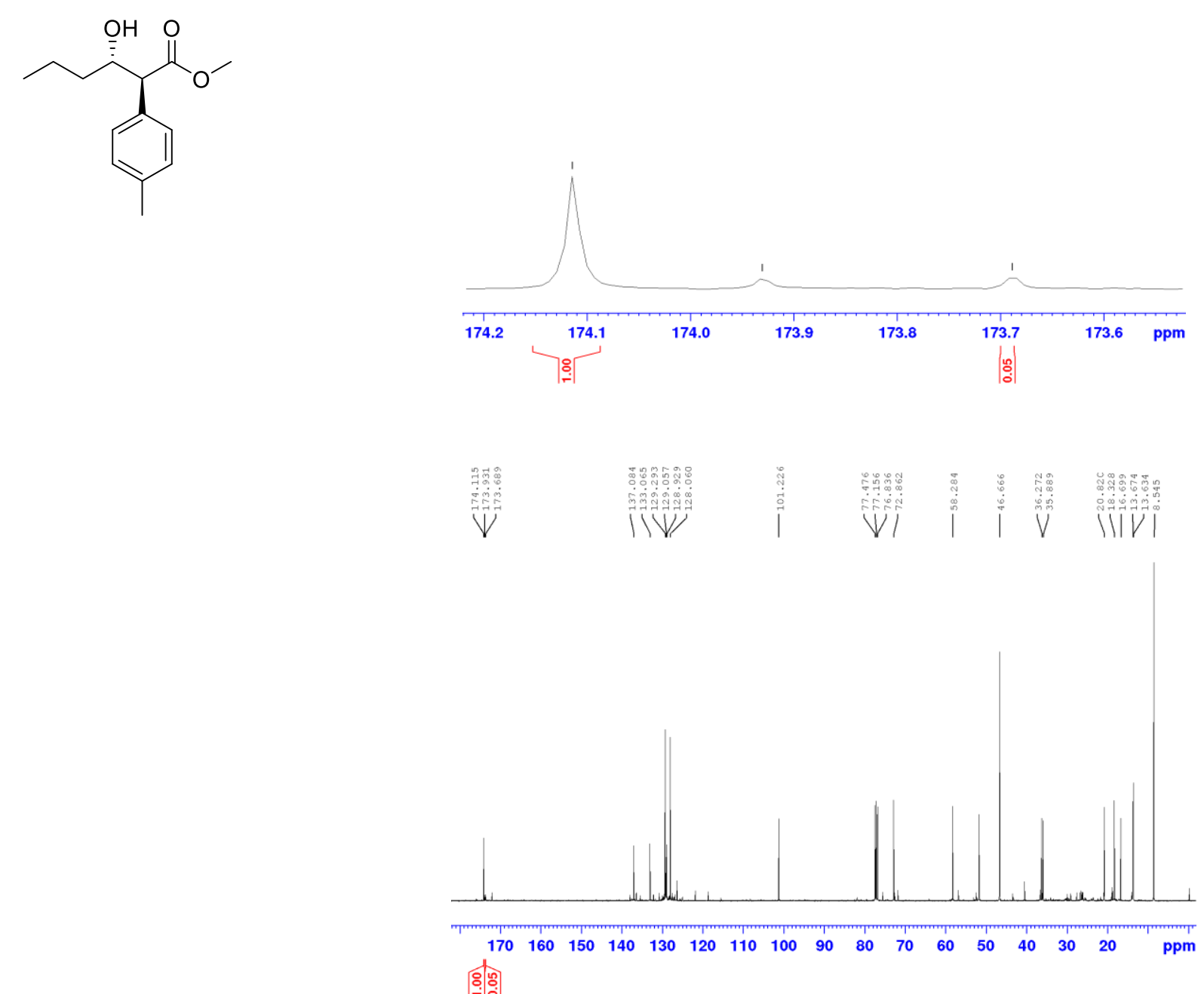

${ }^{13} \mathrm{C}\left\{{ }^{1} \mathrm{H}\right\}$ NMR (100 MHz, $\left.\mathrm{CDCl}_{3}\right)$ Spectrum of crude anti-methyl 3-hydroxy-2-p-tolylhexanoate (7j) 

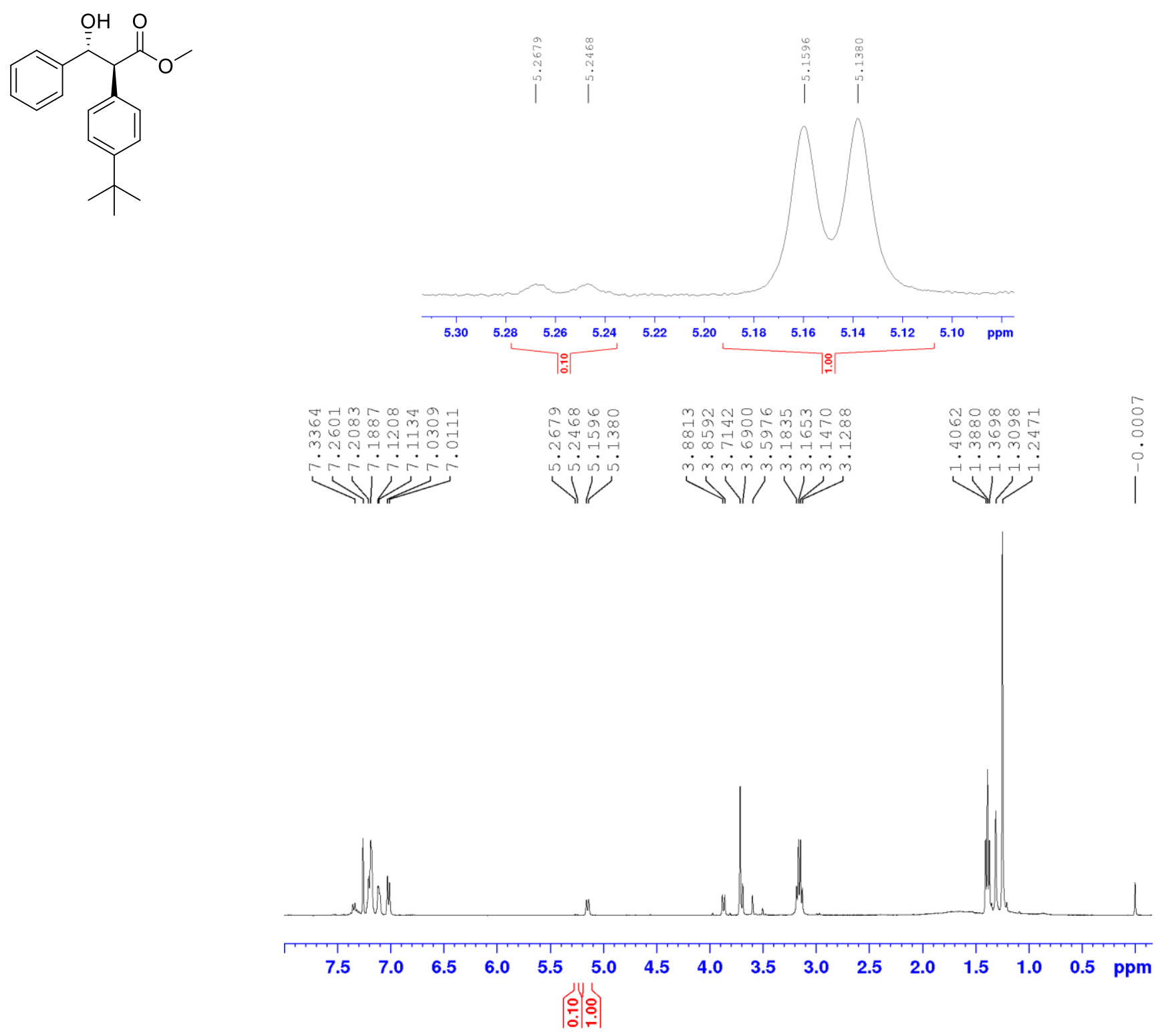

${ }^{1} \mathrm{H}$ NMR (400 MHz, $\mathrm{CDCl}_{3}$ ) Spectrum of crude anti-methyl 2-(4-tert-butylphenyl)-3-hydroxy-3-phenylpropanoate (10j) 

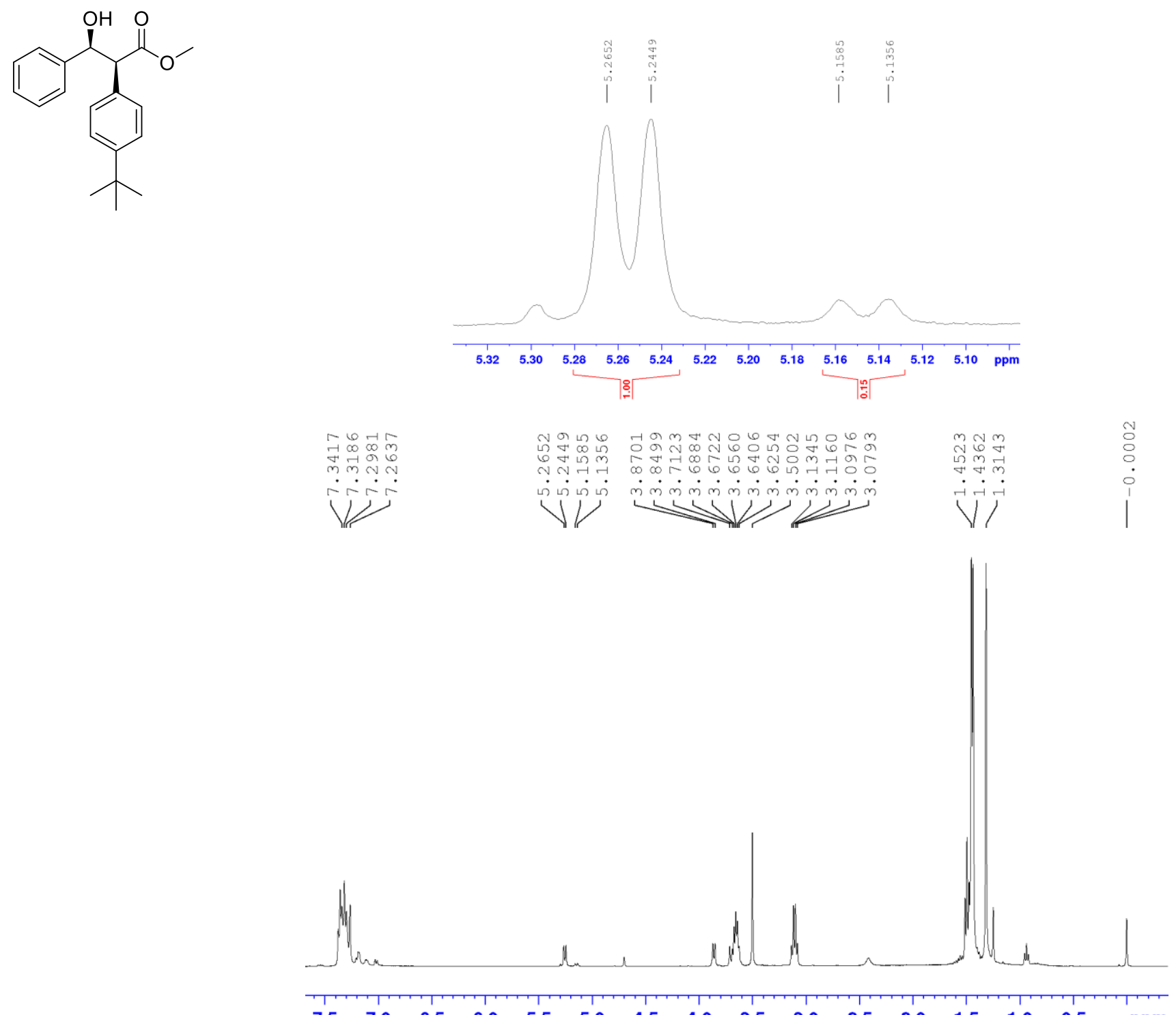
(ํ)

${ }^{1} \mathrm{H}$ NMR (400 MHz, $\mathrm{CDCl}_{3}$ ) Spectrum of crude syn-methyl 2-(4-tert-butylphenyl)-3-hydroxy-3-phenylpropanoate (10j) 